\title{
Double Nucleophilic 1,2-Addition of Silylated Dialkyl Phosphites to 4-Phosphono-1-aza-1,3-dienes: Synthesis of $\boldsymbol{\gamma}$-Phosphono- $\alpha$ - aminobisphosphonates
}

\author{
Kurt G. R. Masschelein and Christian V. Stevens* \\ Research group SynBioC, Department of Organic Chemistry, Faculty of Bioscience \\ Engineering, Ghent University, Coupure Links 653, B-9000 Ghent, Belgium \\ chris.stevens@ugent.be
}

\section{Table of contents}

S2 Microwave reactions

S2 General remarks

S3 Synthesis of 4-phosphono-1-aza-1,3-dienes 1

S6 Synthesis of di(m)ethyl 2,3-epoxypropylphosphonate 3a,b

S7 Synthesis of di(m)ethyl (1E)-3-oxoprop-1-enylphosphonate $\mathbf{4 a , b}$

S8 Synthesis of di(m)ethyl (1E,3E)-3-(dimethylhydrazono)prop-1-enylphosphonate $\mathbf{6 a}, \mathbf{b}$

S8 Synthesis of $\gamma$-phosphono- $\alpha$-aminobisphosphonates 9

S11 ${ }^{1}$ H-NMR and ${ }^{13}$ C-NMR-spectra: 1a-k, 3a-b, 4a-b, 5, 6a-b, 9a-h, 10 
Microwave reactions: all microwave reactions were performed in the CEM Focused Microwave $^{T M}$ Synthesis System, Model Discover with a selectable power output from 0-300 watts. The reactions were performed in $10-\mathrm{mL}$ thick walled Pyrex reaction vessels closed with a Septa cap and equipped with a small stirring bar. The temperature control system uses a non-contact infrared sensor to measure temperature on the bottom of the vessel and is used in a feedback loop with the on-board computer to regulate the temperature from $25-250{ }^{\circ} \mathrm{C}$ by adjusting the power output (1-watt increments). The pressure control, IntelliVent ${ }^{T M}$ Pressure Control System, uses an indirect measurement of the pressure by sensing changes in the external deflection of the septa on the top of the sealed pressure vessel. Stirring is performed by a rotating magnetic plate located below the floor of the microwave cavity. Cooling of the vessel after the reaction is performed by a stream of clean air onto the vessel which decreases the temperature of a $2 \mathrm{~mL}$ solution from $\sim 150{ }^{\circ} \mathrm{C}$ to $\sim 40{ }^{\circ} \mathrm{C}$ in less than 120 seconds. A ramp time of maximum 5 minutes is used during which the temperature increases from RT to the desired temperature. This temperature is maintained during the course of the reaction for the indicated time.

General remarks: High resolution ${ }^{1} \mathrm{H}-\mathrm{NMR}$ spectra were recorded at $300 \mathrm{MHz}$ and ${ }^{13} \mathrm{C}$ NMR spectra at $75 \mathrm{MHz}$. Peak assignments were obtained with the aid of DEPT, 2D-HSQC, 2D-COSY spectra. The compounds were diluted in deuterated solvents and the used solvent is indicated for each compound. Tetramethylsilane or the deuterated solvent were used as internal standards. Mass spectra were obtained with a mass spectrometer (70 eV) using a GCMS coupling (20 m glass capillary column, i.d. $0.53 \mathrm{~mm}, \mathrm{He}$ carrier gas) or were recorded using a direct inlet system $(70 \mathrm{eV})$. Dichloromethane was distilled over calcium hydride. All other solvents were used as received from the supplier. 


\section{Synthesis of 4-phosphono-1-aza-1,3-dienes 1}

\section{Dimethyl (1E,3E)-3-(isopropylimino)prop-1-enylphosphonate 1b}

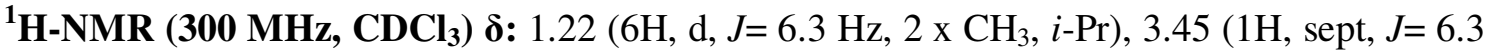
$\mathrm{Hz}, \mathrm{CH}, i-\mathrm{Pr}), 3.77\left(6 \mathrm{H}, \mathrm{d},{ }^{3} J_{\mathrm{HP}}=11.0 \mathrm{~Hz}, 2 \times \mathrm{P}(\mathrm{O}) \mathrm{OCH}_{3}\right), 6.16\left(1 \mathrm{H}, \mathrm{dd},{ }^{2} J_{\mathrm{HP}}=18.4 \mathrm{~Hz}, J=\right.$ $17.3 \mathrm{~Hz}, \mathrm{CHP}), 7.08\left(1 \mathrm{H}\right.$, ddd, $\left.{ }^{3} J_{\mathrm{HP}}=20.6 \mathrm{~Hz}, J=17.3 \mathrm{~Hz}, J=8.5 \mathrm{~Hz}, \underline{\mathrm{HC}}=\mathrm{CHP}\right), 7.97(1 \mathrm{H}, \mathrm{d}$, $J=8.5 \mathrm{~Hz}, \mathrm{HC}=\mathrm{N}) .{ }^{13} \mathbf{C}$-NMR $\left(75 \mathrm{MHz}, \mathbf{C D C l}_{3}\right) \boldsymbol{\delta}: 23.8\left(2 \times \mathrm{CH}_{3}, i\right.$-Pr $), 52.8\left(\mathrm{~d},{ }^{2} J_{\mathrm{CP}}=5.8\right.$ $\mathrm{Hz}, 2$ x $\left.\mathrm{P}(\mathrm{O}) \mathrm{OCH}_{3}\right), 61.7(\mathrm{CH}, i-\mathrm{Pr}), 125.6\left(\mathrm{~d},{ }^{1} J_{\mathrm{CP}}=189.2 \mathrm{~Hz}, \mathrm{CHP}\right), 147.0\left(\mathrm{~d},{ }^{2} J_{\mathrm{CP}}=5.8 \mathrm{~Hz}\right.$, $\mathrm{HC}=\mathrm{CHP}), 158.1\left(\mathrm{~d},{ }^{3} J_{\mathrm{CP}}=30.0 \mathrm{~Hz}, \mathrm{HC}=\mathrm{N}\right) .{ }^{31} \mathbf{P}-\mathbf{N M R}\left(\mathbf{1 2 1} \mathbf{M H z}, \mathbf{C D C l}_{\mathbf{3}}\right) \boldsymbol{\delta}: 19.60 . \mathbf{I R}\left(\mathbf{c m}^{-}\right.$

1) $\mathbf{v}_{\text {max }}$ : 2968, $1600(\mathrm{C}=\mathrm{N}), 1251(\mathrm{br}, \mathrm{P}=\mathrm{O}), 1030(\mathrm{br}, \mathrm{P}-\mathrm{O}) . \mathbf{M S} \boldsymbol{m} / \boldsymbol{z}(\%)$ : (ES, Pos) 206 $\left(\mathrm{M}+\mathrm{H}^{+}, 100\right)$. Elem. Anal. Calcd for $\mathrm{C}_{8} \mathrm{H}_{16} \mathrm{NO}_{3} \mathrm{P}: \mathrm{C} 46.83, \mathrm{H}$ 7.86, N 6.83. Found: $\mathrm{C} 46.56$, H 7.70, N 7.01. Yield: $91 \%$.

\section{Dimethyl (1E,3E)-3-(sec-butylimino)prop-1-enylphosphonate 1c}

${ }^{1}$ H-NMR (300 MHz, CDCl $\left.)_{3}\right) \boldsymbol{\delta}: 0.81\left(3 \mathrm{H}, \mathrm{t}, J=7.4 \mathrm{~Hz}, \mathrm{CH}_{2} \mathrm{CH}_{3}, \sec -\mathrm{Bu}\right), 1.20(3 \mathrm{H}, \mathrm{d}, J=6.3$ $\mathrm{Hz}, \mathrm{CH}_{3}$, sec-Bu), 1.49-1.63 (1H, m, $\underline{\mathrm{C}}_{2} \mathrm{CH}_{3}$, sec- $\left.\mathrm{Bu}\right), 3.12(1 \mathrm{H}$, sext, $J=6.3 \mathrm{~Hz}, \mathrm{NCH}$, sec$\mathrm{Bu}), 3.78\left(6 \mathrm{H}, \mathrm{d},{ }^{3} J_{\mathrm{HP}}=11.0 \mathrm{~Hz}, 2 \times \mathrm{P}(\mathrm{O}) \mathrm{OCH}_{3}\right), 6.17\left(1 \mathrm{H}, \mathrm{dd},{ }^{2} J_{\mathrm{HP}}=17.9 \mathrm{~Hz}, J=17.3 \mathrm{~Hz}\right.$, CHP), $7.10\left(1 \mathrm{H}, \mathrm{ddd},{ }^{3} J_{\mathrm{HP}}=20.9 \mathrm{~Hz}, J=17.3 \mathrm{~Hz}, J=8.8 \mathrm{~Hz}, \underline{\mathrm{HC}}=\mathrm{CHP}\right), 7.94(1 \mathrm{H}, \mathrm{d}, J=8.8$

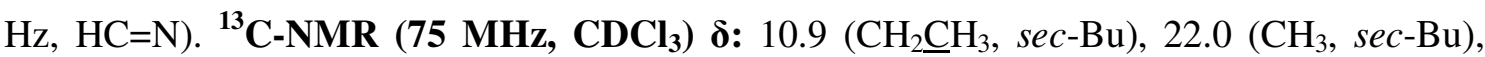
$30.4\left(\mathrm{CH}_{2} \mathrm{CH}_{3}\right.$, sec-Bu), $52.7\left(\mathrm{~d},{ }^{2} J_{\mathrm{CP}}=5.8 \mathrm{~Hz}, 2 \times \mathrm{P}(\mathrm{O}) \mathrm{OCH}_{3}\right), 68.3(\mathrm{NCH}$, sec-Bu $), 125.5(\mathrm{~d}$, $\left.{ }^{1} J_{\mathrm{CP}}=190.4 \mathrm{~Hz}, \mathrm{CHP}\right), 146.8\left(\mathrm{~d},{ }^{2} J_{\mathrm{CP}}=5.8 \mathrm{~Hz}, \mathrm{HC}=\mathrm{CHP}\right), 158.6\left(\mathrm{~d},{ }^{3} J_{\mathrm{CP}}=30.0 \mathrm{~Hz}, \mathrm{HC}=\mathrm{N}\right)$.

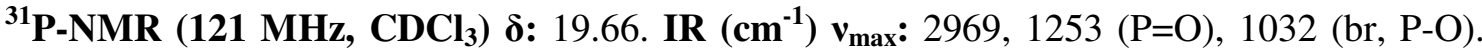
MS $m / z$ (\%): (ES, Pos) $220\left(\mathrm{M}+\mathrm{H}^{+}, 100\right)$. Elem. Anal. Calcd for $\mathrm{C}_{9} \mathrm{H}_{18} \mathrm{NO}_{3} \mathrm{P}: \mathrm{C} 49.31, \mathrm{H}$ 8.28, N 6.39. Found: C 49.10, H 8.47, N 6.43. Yield: $99 \%$.

\section{Dimethyl (1E,3E)-3-(isobutylimino)prop-1-enylphosphonate 1d}

${ }^{1}$ H-NMR (300 MHz, CDCl $)$ ) $0.93(6 \mathrm{H}, \mathrm{d}, J=6.6 \mathrm{~Hz}, 2$ x CH,$i-\mathrm{Bu}), 1.98$ (1H, non, $J=6.6$ $\mathrm{Hz}, \mathrm{CH}, i-\mathrm{Bu}), 3.37\left(2 \mathrm{H}, \mathrm{d}, J=6.6 \mathrm{~Hz}, \mathrm{NCH}_{2}, i-\mathrm{Bu}\right), 3.78\left(6 \mathrm{H},{ }^{3} J_{\mathrm{HP}}=11.0 \mathrm{~Hz}, 2 \mathrm{x}\right.$ $\left.\mathrm{P}(\mathrm{O}) \mathrm{OCH}_{3}\right), 6.16\left(1 \mathrm{H}, \mathrm{dd},{ }^{2} J_{\mathrm{HP}}=18.2 \mathrm{~Hz}, J=17.3 \mathrm{~Hz}, \mathrm{CHP}\right), 7.11\left(1 \mathrm{H}, \mathrm{ddd},{ }^{3} J_{\mathrm{HP}}=20.6 \mathrm{~Hz}, J=\right.$ $17.3 \mathrm{~Hz}, J=8.5 \mathrm{~Hz}, \underline{\mathrm{HC}}=\mathrm{CHP}), 7.92\left(1 \mathrm{H}, \mathrm{dd}, J=8.5 \mathrm{~Hz},{ }^{4} J_{\mathrm{HP}}=1.4 \mathrm{~Hz}, \mathrm{HC}=\mathrm{N}\right) .{ }^{\mathbf{1 3}} \mathbf{C}-\mathbf{N M R}(\mathbf{7 5}$ MHz, $\left.\mathbf{C D C l}_{3}\right)$ 反: $20.6\left(2 \times \mathrm{CH}_{3}, i-\mathrm{Bu}\right), 29.4(\mathrm{CH}, i-\mathrm{Bu}), 52.7\left(\mathrm{~d},{ }^{2} J_{\mathrm{CP}}=5.8 \mathrm{~Hz}, 2 \times \mathrm{P}(\mathrm{O}) \mathrm{OMe}\right)$, $69.9(\mathrm{NCH}, i-\mathrm{Bu}), 125.5\left(\mathrm{~d},{ }^{1} J_{\mathrm{CP}}=190.4 \mathrm{~Hz}, \mathrm{CHP}\right), 146.8\left(\mathrm{~d},{ }^{2} J_{\mathrm{CP}}=5.8 \mathrm{~Hz}, \mathrm{HC}=\mathrm{CHP}\right), 160.6$

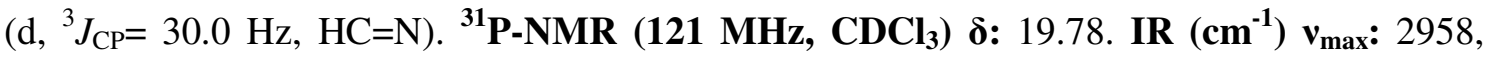


$1247(\mathrm{P}=\mathrm{O}), 1031$ (br, P-O). MS m/z (\%): (ES, Pos) $220\left(\mathrm{M}+\mathrm{H}^{+}, 100\right)$. Elem. Anal. Calcd for $\mathrm{C}_{9} \mathrm{H}_{18} \mathrm{NO}_{3} \mathrm{P}: \mathrm{C} 49.31, \mathrm{H}$ 8.28, N 6.39. Found: C 49.34, H 8.34, N 6.55. Yield: $91 \%$.

\section{Dimethyl (1E,3E)-3-(cyclohexylimino)prop-1-enylphosphonate 1e}

${ }^{1}$ H-NMR (300 MHz, CDCl $)$ א: 1.16-1.84 (10H, m, 5 x CH $\mathrm{NCH}, c-\mathrm{Hex}), 3.77\left(6 \mathrm{H}, \mathrm{d},{ }^{3} J_{\mathrm{HP}}=11.0 \mathrm{~Hz}, 2 \times \mathrm{P}(\mathrm{O}) \mathrm{OMe}\right), 6.15\left(1 \mathrm{H}, \mathrm{dd},{ }^{2} J_{\mathrm{HP}}=18.4 \mathrm{~Hz}, J=\right.$ $17.3 \mathrm{~Hz}, \mathrm{CHP}), 7.09\left(1 \mathrm{H}, \mathrm{ddd},{ }^{3} J_{\mathrm{HP}}=20.6 \mathrm{~Hz}, J=17.3 \mathrm{~Hz}, J=8.8 \mathrm{~Hz}, \underline{\mathrm{HC}}=\mathrm{CHP}\right), 7.98(1 \mathrm{H}$, $\left.\mathrm{dd}, J=8.8 \mathrm{~Hz},{ }^{4} J_{\mathrm{HP}}=1.4 \mathrm{~Hz}, \mathrm{HC}=\mathrm{N}\right) .{ }^{13} \mathbf{C}-\mathbf{N M R}\left(\mathbf{7 5} \mathbf{M H z}, \mathbf{C D C l}_{3}\right) \boldsymbol{\delta}: 24.5\left(2 \mathrm{x} \mathrm{CH}_{2}, c-\mathrm{Hex}\right)$, $25.5\left(\mathrm{CH}_{2}, c\right.$-Hex), $34.0\left(2 \times \mathrm{CH}_{2}, c-\mathrm{Hex}\right), 52.8\left(\mathrm{~d},{ }^{2} J_{\mathrm{CP}}=5.8 \mathrm{~Hz}, 2 \times \mathrm{P}(\mathrm{O}) \mathrm{OMe}\right), 70.0(\mathrm{NCH}$, $c$-Hex), $125.4\left(\mathrm{~d},{ }^{1} J_{\mathrm{CP}}=189.2 \mathrm{~Hz}, \mathrm{CHP}\right), 147.0\left(\mathrm{~d},{ }^{2} J_{\mathrm{CP}}=5.8 \mathrm{~Hz}, \mathrm{HC}=\mathrm{CHP}\right), 158.5\left(\mathrm{~d},{ }^{3} J_{\mathrm{CP}}=\right.$

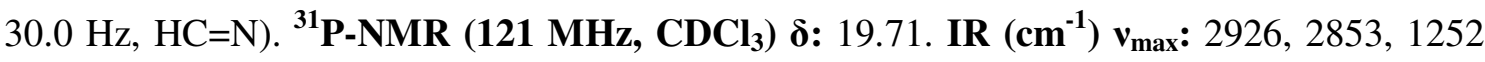
$(\mathrm{P}=\mathrm{O}), 1061$ (P-O), 1038 (P-O). MS m/z (\%): (ES, Pos) $246\left(\mathrm{M}+\mathrm{H}^{+}, 100\right)$. Mp.: 38-39 ${ }^{\circ} \mathrm{C}$. Elem. Anal. Calcd for $\mathrm{C}_{11} \mathrm{H}_{20} \mathrm{NO}_{3} \mathrm{P}$ : C 53.87, H 8.22, N 5.71. Found: C 53.78, H 8.34, N 5.64. Yield: $99 \%$.

\section{Dimethyl (1E,3E)-3-(benzylimino)prop-1-enylphosphonate $1 f$}

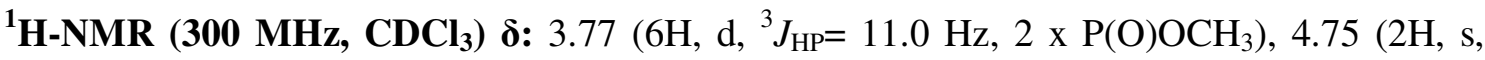
$\left.\mathrm{NCH}_{2} \mathrm{Ph}\right), 6.21\left(1 \mathrm{H}, \mathrm{dd},{ }^{2} J_{\mathrm{HP}}=18.2 \mathrm{~Hz}, J=17.3 \mathrm{~Hz}, \mathrm{CHP}\right), 7.16\left(1 \mathrm{H}, \mathrm{ddd},{ }^{3} J_{\mathrm{HP}}=20.6 \mathrm{~Hz}, J=\right.$ $17.3 \mathrm{~Hz}, J=8.5 \mathrm{~Hz}, \underline{\mathrm{HC}}=\mathrm{CHP}), 7.26-7.38\left(5 \mathrm{H}, \mathrm{m}, 5\right.$ x $\left.\mathrm{CH}_{\text {arom }}\right), 8.06(1 \mathrm{H}, \mathrm{dd}, J=8.5 \mathrm{~Hz}, J=$ $1.1 \mathrm{~Hz}, \mathrm{HC}=\mathrm{N}) .{ }^{13} \mathbf{C}-\mathbf{N M R}\left(\mathbf{7 5} \mathbf{M H z}, \mathbf{C D C l}_{3}\right) \boldsymbol{\delta}: 52.8\left(\mathrm{~d},{ }^{2} J_{\mathrm{CP}}=5.8 \mathrm{~Hz}, 2\right.$ x P(O)OCH$), 65.3$ $\left(\mathrm{NCH}_{2} \mathrm{Ph}\right), 126.3\left(\mathrm{~d},{ }^{1} J_{\mathrm{CP}}=189.2 \mathrm{~Hz}, \mathrm{CHP}\right), 127.4\left(\mathrm{CH}_{\text {arom }}\right), 128.1\left(2 \times \mathrm{CH}_{\text {arom }}\right), 128.7$ (2 x $\left.\mathrm{CH}_{\text {arom }}\right), 138.1\left(\mathrm{C}_{\text {quat,arom }}\right), 146.6\left(\mathrm{~d},{ }^{2} J_{\mathrm{CP}}=5.8 \mathrm{~Hz}, \mathrm{HC}=\mathrm{CHP}\right), 161.4\left(\mathrm{~d},{ }^{3} J_{\mathrm{CP}}=30.0 \mathrm{~Hz}\right.$,

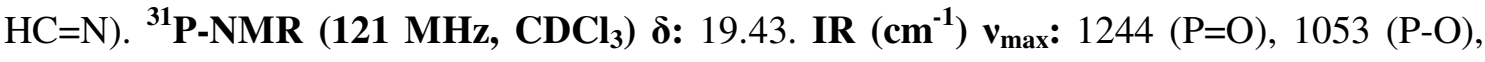

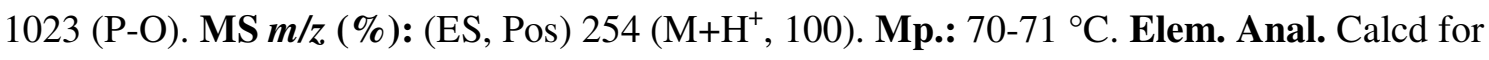
$\mathrm{C}_{12} \mathrm{H}_{16} \mathrm{NO}_{3} \mathrm{P}: \mathrm{C} 56.92, \mathrm{H}$ 6.37, N 5.53. Found: C 56.66, H 6.18, N 5.51. Yield: $97 \%$.

\section{Diethyl (1E,3E)-3-(t-butylimino)prop-1-enylphosphonate $1 \mathrm{~g}$}

${ }^{1}$ H-NMR (300 MHz, CDCl $)$ א: $1.24\left(9 \mathrm{H}, \mathrm{s}, 3 \times \mathrm{CH}_{3}, t\right.$-Bu), $1.35(6 \mathrm{H}, \mathrm{t}, J=7.0 \mathrm{~Hz}, 2 \mathrm{x}$ $\left.\mathrm{P}(\mathrm{O}) \mathrm{OCH}_{2} \mathrm{C}_{3}\right), 4.09-4.18\left(4 \mathrm{H}, \mathrm{m}, 2 \times \mathrm{P}(\mathrm{O}) \mathrm{OC}_{2} \underline{\mathrm{H}}_{3}\right), 6.21\left(1 \mathrm{H}, \mathrm{dd},{ }^{2} J_{\mathrm{HP}}=18.3 \mathrm{~Hz}, J=17.2\right.$ $\mathrm{Hz}, \mathrm{CHP}), 7.09\left(1 \mathrm{H}, \mathrm{ddd},{ }^{3} J_{\mathrm{HP}}=20.5 \mathrm{~Hz}, J=17.2 \mathrm{~Hz}, J=8.5 \mathrm{~Hz}, \underline{\mathrm{HC}}=\mathrm{CHP}\right), 7.94(1 \mathrm{H}, \mathrm{d}, J=$ $8.5 \mathrm{~Hz}, \mathrm{HC}=\mathrm{N}) .{ }^{13} \mathbf{C}-\mathrm{NMR}\left(\mathbf{7 5} \mathbf{M H z}, \mathbf{C D C l}_{3}\right) \boldsymbol{\delta}: 16.4\left(\mathrm{~d},{ }^{3} J_{\mathrm{CP}}=5.8 \mathrm{~Hz}, 2 \mathrm{x} \mathrm{P}(\mathrm{O}) \mathrm{OCH}_{2} \mathrm{CH}_{3}\right)$, $29.4\left(3 \times \mathrm{CH}_{3}, t-\mathrm{Bu}\right), 58.2\left(\mathrm{C}_{\text {quat }}, t-\mathrm{Bu}\right), 62.2\left(\mathrm{~d},{ }^{2} J_{\mathrm{CP}}=5.8 \mathrm{~Hz}, 2 \times \mathrm{P}(\mathrm{O}) \mathrm{OCH}_{2} \mathrm{CH}_{3}\right), 126.8(\mathrm{~d}$, $\left.{ }^{1} J_{\mathrm{CP}}=189.2 \mathrm{~Hz}, \mathrm{CHP}\right), 146.9\left(\mathrm{~d},{ }^{2} J_{\mathrm{CP}}=4.7 \mathrm{~Hz}, \mathrm{HC}=\mathrm{CHP}\right), 155.7\left(\mathrm{~d},{ }^{3} J_{\mathrm{CP}}=30.0 \mathrm{~Hz}, \mathrm{HC}=\mathrm{N}\right)$.

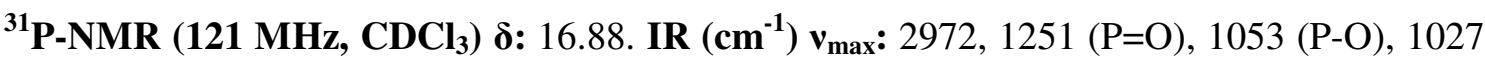


(P-O). MS $m / z(\%)$ : (ES, Pos) $248\left(\mathrm{M}+\mathrm{H}^{+}, 100\right)$. Elem. Anal. Calcd for $\mathrm{C}_{11} \mathrm{H}_{22} \mathrm{NO}_{3} \mathrm{P}$ : C 53.43, H 8.97, N 5.66. Found: C 53.70, H 8.99, N 5.46. Yield: $93 \%$.

\section{Diethyl (1E,3E)-3-(isopropylimino)prop-1-enylphosphonate $1 \mathrm{~h}$}

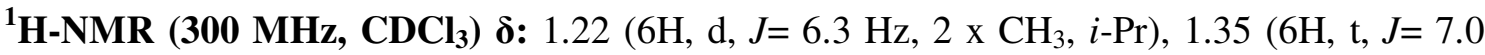
$\left.\mathrm{Hz}, 2 \times \mathrm{P}(\mathrm{O}) \mathrm{OCH}_{2} \mathrm{CH}_{3}\right), 3.44(1 \mathrm{H}$, sept, $J=6.3 \mathrm{~Hz}, \mathrm{CH}, i-\mathrm{Pr}), 4.13\left(4 \mathrm{H}, \mathrm{dq},{ }^{3} J_{\mathrm{HP}}=7.2 \mathrm{~Hz}, J=\right.$ $\left.7.0 \mathrm{~Hz}, 2 \times \mathrm{P}(\mathrm{O}) \mathrm{OC}_{2} \mathrm{CH}_{3}\right), 6.19\left(1 \mathrm{H}, \mathrm{dd},{ }^{2} J_{\mathrm{HP}}=17.9 \mathrm{~Hz}, J=17.6 \mathrm{~Hz}, \mathrm{CHP}\right), 7.06(1 \mathrm{H}, \mathrm{ddd}$, $\left.{ }^{3} J_{\mathrm{HP}}=20.7 \mathrm{~Hz}, J=17.6 \mathrm{~Hz}, J=8.8 \mathrm{~Hz}, \underline{\mathrm{HC}}=\mathrm{CHP}\right), 7.96(1 \mathrm{H}, \mathrm{d}, J=8.8 \mathrm{~Hz}, \mathrm{HC}=\mathrm{N}) .{ }^{\mathbf{1 3}} \mathbf{C}-\mathbf{N M R}$

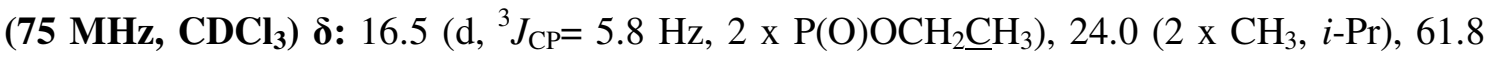
$\left(\mathrm{CH}, i\right.$-Pr), $62.3\left(\mathrm{~d},{ }^{2} J_{\mathrm{CP}}=5.8 \mathrm{~Hz}, 2 \times \mathrm{P}(\mathrm{O}) \mathrm{OCH}_{2} \mathrm{CH}_{3}\right), 127.2\left(\mathrm{~d},{ }^{1} J_{\mathrm{CP}}=189.2 \mathrm{~Hz}, \mathrm{CHP}\right), 146.1$ $\left(\mathrm{d},{ }^{2} J_{\mathrm{CP}}=4.6 \mathrm{~Hz}, \mathrm{HC}=\mathrm{CHP}\right), 158.4\left(\mathrm{~d},{ }^{3} J_{\mathrm{CP}}=30.0 \mathrm{~Hz}, \mathrm{HC}=\mathrm{N}\right) .{ }^{31} \mathbf{P}-\mathbf{N M R}\left(\mathbf{1 2 1} \mathbf{M H z}, \mathbf{C D C l}_{\mathbf{3}}\right)$

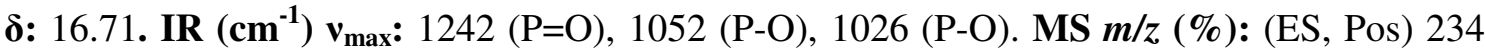
$\left(\mathrm{M}+\mathrm{H}^{+}, 100\right)$. Elem. Anal. Calcd for $\mathrm{C}_{10} \mathrm{H}_{20} \mathrm{NO}_{3} \mathrm{P}: \mathrm{C}$ 51.49, H 8.64, N 6.01. Found: C 51.57, H 8.65, N 5.99. Yield: $99 \%$.

\section{Diethyl (1E,3E)-3-(sec-butylimino)prop-1-enylphosphonate 1i}

${ }^{1} \mathbf{H}-\mathrm{NMR}\left(\mathbf{3 0 0} \mathbf{M H z}, \mathbf{C D C l}_{3}\right) \boldsymbol{\delta}: 0.81\left(3 \mathrm{H}, \mathrm{t}, J=7.3 \mathrm{~Hz}, \mathrm{CH}_{2} \mathrm{CH}_{3}, \sec -\mathrm{Bu}\right), 1.20(3 \mathrm{H}, \mathrm{d}, J=6.3$ $\mathrm{Hz}, \mathrm{CH}_{3}$, sec-Bu $), 1.35\left(6 \mathrm{H}, \mathrm{t}, J=7.0 \mathrm{~Hz}, 2\right.$ x $\left.\mathrm{P}(\mathrm{O}) \mathrm{OCH}_{2} \mathrm{C}_{3}\right), 1.57(1 \mathrm{H}, \mathrm{dq}, J=7.3 \mathrm{~Hz}, J=6.3$ $\mathrm{Hz}, \underline{\mathrm{C}}_{2} \mathrm{CH}_{3}$, sec-Bu), $3.10(1 \mathrm{H}$, sext, $J=6.3 \mathrm{~Hz}, \mathrm{NCH}$, sec-Bu$), 4.14\left(4 \mathrm{H}, \mathrm{dq},{ }^{3} J_{\mathrm{HP}}=7.2 \mathrm{~Hz}\right.$, $\left.J=7.0 \mathrm{~Hz}, 2 \times \mathrm{P}(\mathrm{O}) \mathrm{OCH}_{2} \mathrm{CH}_{3}\right), 6.19\left(1 \mathrm{H}, \mathrm{dd},{ }^{2} J_{\mathrm{HP}}=18.2 \mathrm{~Hz}, J=17.3 \mathrm{~Hz}, \mathrm{CHP}\right), 7.07(1 \mathrm{H}$, ddd, $\left.{ }^{3} J_{\mathrm{HP}}=20.9 \mathrm{~Hz}, J=17.3 \mathrm{~Hz}, J=8.8 \mathrm{~Hz}, \underline{\mathrm{HC}}=\mathrm{CHP}\right), 7.92(1 \mathrm{H}, \mathrm{d}, J=8.8 \mathrm{~Hz}, \mathrm{HC}=\mathrm{N}) .{ }^{13} \mathrm{C}-$ NMR (75 MHz, $\left.\mathbf{C D C l}_{3}\right)$ \&: $10.9\left(\mathrm{CH}_{2} \mathrm{CH}_{3}\right.$, sec-Bu), $16.4\left(\mathrm{~d},{ }^{3} J_{\mathrm{CP}}=5.8 \mathrm{~Hz}, 2 \mathrm{x}\right.$ $\left.\mathrm{P}(\mathrm{O}) \mathrm{OCH}_{2} \underline{\mathrm{CH}}_{3}\right), 22.0\left(\mathrm{CH}_{3}\right.$, sec-Bu $), 30.5\left(\mathrm{CH}_{2} \mathrm{CH}_{3}\right.$, sec $\left.-\mathrm{Bu}\right), 62.3\left(\mathrm{~d},{ }^{2} J_{\mathrm{CP}}=5.8 \mathrm{~Hz}, 2 \mathrm{x}\right.$ $\left.\mathrm{P}(\mathrm{O}) \mathrm{OCH}_{2} \mathrm{CH}_{3}\right), 68.4\left(\mathrm{NCH}\right.$, sec-Bu), 127.1 (d, $\left.{ }^{1} J_{\mathrm{CP}}=189.2 \mathrm{~Hz}, \mathrm{CHP}\right), 145.9\left(\mathrm{~d},{ }^{2} J_{\mathrm{CP}}=5.8\right.$ $\mathrm{Hz}, \mathrm{HC}=\mathrm{CHP}), 158.9\left(\mathrm{~d},{ }^{3} J_{\mathrm{CP}}=30.0 \mathrm{~Hz}, \mathrm{HC}=\mathrm{N}\right) .{ }^{31} \mathbf{P}-\mathbf{N M R}\left(\mathbf{1 2 1} \mathbf{M H z}, \mathbf{C D C l}_{\mathbf{3}}\right) \boldsymbol{\delta}: 16.80 . \mathbf{I R}$ $\left(\mathbf{c m}^{-1}\right) v_{\text {max }}$ : 2970, 2930, 1245 (P=O), 1053 (P-O), 1027 (P-O). MS $\boldsymbol{m} / \boldsymbol{z}$ (\%): (ES, Pos) 248 $\left(\mathrm{M}+\mathrm{H}^{+}, 100\right)$. Elem. Anal. Calcd for $\mathrm{C}_{11} \mathrm{H}_{22} \mathrm{NO}_{3} \mathrm{P}: \mathrm{C}$ 53.43, H 8.97, N 5.66. Found: C 53.23, H 8.87, N 5.77. Yield: $97 \%$.

\section{Diethyl (1E,3E)-3-(isobutylimino)prop-1-enylphosphonate $1 \mathbf{j}$}

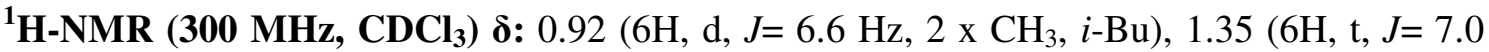
$\left.\mathrm{Hz}, 2 \times \mathrm{P}(\mathrm{O}) \mathrm{OCH}_{2} \mathrm{C}_{3}\right), 1.97(1 \mathrm{H}$, non, $J=6.6 \mathrm{~Hz}, \mathrm{CH}, i-\mathrm{Bu}), 3.36\left(2 \mathrm{H}, \mathrm{d}, J=6.6 \mathrm{~Hz}, \mathrm{NCH}_{2}\right.$, $i$-Bu $), 4.14\left(4 \mathrm{H}, \mathrm{dq},{ }^{3} J_{\mathrm{HP}}=7.4 \mathrm{~Hz}, J=7.0 \mathrm{~Hz}, 2 \times \mathrm{P}(\mathrm{O}) \mathrm{OCH}_{2} \mathrm{CH}_{3}\right), 6.19\left(1 \mathrm{H}, \mathrm{dd},{ }^{2} J_{\mathrm{HP}}=17.9\right.$ $\mathrm{Hz}, J=17.3 \mathrm{~Hz}, \mathrm{CHP}), 7.08\left(1 \mathrm{H}, \mathrm{ddd},{ }^{3} J_{\mathrm{HP}}=20.6 \mathrm{~Hz}, J=17.3 \mathrm{~Hz}, J=8.8 \mathrm{~Hz}, \underline{\mathrm{HC}}=\mathrm{CHP}\right), 7.92$ 
$\left(1 \mathrm{H}, \mathrm{dd}, J=8.8 \mathrm{~Hz},{ }^{4} J_{\mathrm{HP}}=1.1 \mathrm{~Hz}, \mathrm{HC}=\mathrm{N}\right) .{ }^{\mathbf{1 3}} \mathbf{C}-\mathbf{N M R}\left(\mathbf{7 5} \mathbf{M H z}, \mathbf{C D C l}_{\mathbf{3}}\right) \boldsymbol{\delta}: 16.4\left(\mathrm{~d},{ }^{3} J_{\mathrm{CP}}=6.9\right.$ $\left.\mathrm{Hz}, 2 \times \mathrm{P}(\mathrm{O}) \mathrm{OCH}_{2} \underline{\mathrm{CH}}_{3}\right), 20.6\left(2 \times \mathrm{CH}_{3}, i-\mathrm{Bu}\right), 29.4(\mathrm{CH}, i-\mathrm{Bu}), 62.3\left(\mathrm{~d},{ }^{2} J_{\mathrm{CP}}=5.8 \mathrm{~Hz}, 2 \mathrm{x}\right.$ $\left.\mathrm{P}(\mathrm{O}) \mathrm{OCH}_{2} \mathrm{CH}_{3}\right), 70.0(\mathrm{NCH}, i-\mathrm{Bu}), 127.1\left(\mathrm{~d},{ }^{1} J_{\mathrm{CP}}=189.2 \mathrm{~Hz}, \mathrm{CHP}\right), 145.9\left(\mathrm{~d},{ }^{2} J_{\mathrm{CP}}=5.8 \mathrm{~Hz}\right.$, HC $=$ CHP $), 160.9\left(\mathrm{~d},{ }^{3} J_{\mathrm{CP}}=30.0 \mathrm{~Hz}, \mathrm{HC}=\mathrm{N}\right) .{ }^{31} \mathbf{P}-\mathbf{N M R}\left(\mathbf{1 2 1} \mathbf{M H z}, \mathbf{C D C l}_{\mathbf{3}}\right) \boldsymbol{\delta}: 16.90 . \mathbf{I R}\left(\mathbf{c m}^{-}\right.$

1) $v_{\text {max }}$ : 2958, 2927, $1247(\mathrm{P}=\mathrm{O}), 1051(\mathrm{P}-\mathrm{O}), 1027$ (P-O). MS $\boldsymbol{m} / \boldsymbol{z}(\boldsymbol{\%})$ : (ES, Pos) 248 $\left(\mathrm{M}+\mathrm{H}^{+}, 100\right)$. Elem. Anal. Calcd for $\mathrm{C}_{11} \mathrm{H}_{22} \mathrm{NO}_{3} \mathrm{P}: \mathrm{C} 53.43$, H 8.97, N 5.66. Found: C 53.39, H 8.99, N 5.85. Yield: 99\%.

\section{Diethyl (1E,3E)-3-(cyclohexylimino)prop-1-enylphosphonate 1k}

${ }^{1}$ H-NMR (300 MHz, CDCl $)$ ): 1.20-1.82 (10H, m, 5 x CH $\left.2, c-H e x\right), 1.35$ (6H, t, J=6.6 Hz, $\left.2 \times \mathrm{P}(\mathrm{O}) \mathrm{OCH}_{2} \mathrm{CH}_{3}\right), 3.12(1 \mathrm{H}, \mathrm{t}, J=9.6 \mathrm{~Hz}, \mathrm{NCH}, c-\mathrm{Hex}), 4.12\left(4 \mathrm{H}, \mathrm{dq},{ }^{3} J_{\mathrm{HP}}=6.6 \mathrm{~Hz}, J=6.6\right.$ $\left.\mathrm{Hz}, 2 \times \mathrm{P}(\mathrm{O}) \mathrm{OC}_{2} \mathrm{CH}_{3}\right), 6.22\left(1 \mathrm{H}, \mathrm{dd}, J=17.9 \mathrm{~Hz},{ }^{2} J_{\mathrm{HP}}=17.6 \mathrm{~Hz}, \mathrm{CHP}\right), 7.09\left(1 \mathrm{H}, \mathrm{ddd},{ }^{3} J_{\mathrm{HP}}=\right.$ $19.8 \mathrm{~Hz}, J=17.9 \mathrm{~Hz}, J=8.3 \mathrm{~Hz}, \underline{\mathrm{HC}}=\mathrm{CHP}), 8.01(1 \mathrm{H}, \mathrm{dd}, J=8.3 \mathrm{~Hz}, \mathrm{HC}=\mathrm{N}) .{ }^{13} \mathbf{C}-\mathbf{N M R}(\mathbf{7 5}$

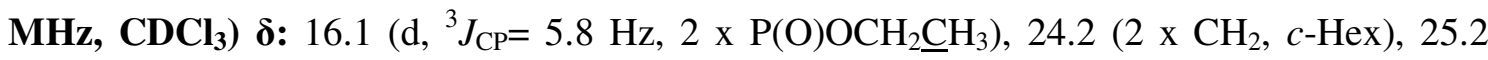
$\left(\mathrm{CH}_{2}, c-\mathrm{Hex}\right), 33.8\left(2 \times \mathrm{CH}_{2}, c-\mathrm{Hex}\right), 61.8\left(\mathrm{~d},{ }^{2} J_{\mathrm{CP}}=5.8 \mathrm{~Hz}, 2 \times \mathrm{P}(\mathrm{O}) \mathrm{OCH}_{2} \mathrm{CH}_{3}\right), 69.6(\mathrm{NCH}$, $c$-Hex), $126.8\left(\mathrm{~d},{ }^{1} J_{\mathrm{CP}}=189.2 \mathrm{~Hz}, \mathrm{CHP}\right), 146.1\left(\mathrm{~d},{ }^{2} J_{\mathrm{CP}}=5.8 \mathrm{~Hz}, \mathrm{HC}=\mathrm{CHP}\right), 158.4\left(\mathrm{~d},{ }^{3} J_{\mathrm{CP}}=\right.$ $30.0 \mathrm{~Hz}, \mathrm{HC}=\mathrm{N}) .{ }^{31} \mathbf{P}-\mathbf{N M R}\left(\mathbf{1 2 1} \mathbf{M H z}, \mathbf{C D C l}_{\mathbf{3}}\right) \boldsymbol{\delta}: \mathbf{1 6 . 7 9}$. IR $\left(\mathbf{c m}^{\mathbf{- 1}}\right) \mathbf{v}_{\max }: 2982,2930,2855$,

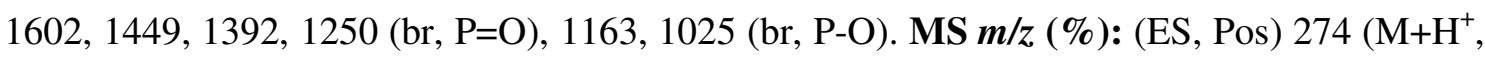
100). Elem. Anal. Calcd for $\mathrm{C}_{13} \mathrm{H}_{24} \mathrm{NO}_{3} \mathrm{P}: \mathrm{C} 57.13$, H 8.85, N 5.12. Found: C 57.25, H 8.96, N 5.16. Yield: $99 \%$.

Synthesis of di(m)ethyl 2,3-epoxypropylphosphonate 3a,b (see Griffin, C. E.; Kundu, S. K. J. Org. Chem. 1969, 34, 1532 for the preparation and spectral data of diethyl 2,3epoxypropylphosphonate $\mathbf{3 b}$ )

A mixture of epibromohydrin $(0,10 \mathrm{~mol})$ and tri $(\mathrm{m})$ ethyl phosphite (1.0 equiv) was heated to $130{ }^{\circ} \mathrm{C}$ under a nitrogen atmosphere, in a flask equiped with Vigreux column and a condensor. At this temperature bromo(m)ethane distilled off; the mixture was then heated at $130{ }^{\circ} \mathrm{C}$ for $4 \mathrm{~h}$ and at $150{ }^{\circ} \mathrm{C}$ for $1 \mathrm{~h}$. The crude product was purified by distillation.

\section{Dimethyl 2,3-epoxypropylphosphonate 3a}

${ }^{1}$ H-NMR (300 MHz, CDCl $)$ א: $1.93\left(1 \mathrm{H}, \mathrm{ddd},{ }^{2} J_{\mathrm{HP}}=20.1 \mathrm{~Hz}, J=15.4 \mathrm{~Hz}, J=6.3 \mathrm{~Hz}\right.$, $\left.\mathrm{C}_{\mathrm{H}_{\mathrm{A}}} \mathrm{H}_{\mathrm{B}} \mathrm{P}\right), 2.20\left(1 \mathrm{H}, \mathrm{ddd},{ }^{2} J_{\mathrm{HP}}=18.4 \mathrm{~Hz}, J=15.4 \mathrm{~Hz}, J=6.1 \mathrm{~Hz}, \mathrm{CH}_{\mathrm{A}} \underline{\mathrm{H}}_{\mathrm{B}} \mathrm{P}\right), 2.60(1 \mathrm{H}, \mathrm{dd}, J=$ $\left.4.7 \mathrm{~Hz}, J=2.5 \mathrm{~Hz}, \underline{\mathrm{C}}_{\mathrm{A}} \mathrm{H}_{\mathrm{B}} \mathrm{O}\right), 2.86\left(1 \mathrm{H}, \mathrm{ddd}, J=4.7 \mathrm{~Hz}, J=3.9 \mathrm{~Hz},{ }^{4} J_{\mathrm{HP}}=1.7 \mathrm{~Hz}, \mathrm{CH}_{\mathrm{A}} \underline{\mathrm{H}}_{\mathrm{B}} \mathrm{O}\right)$, $3.19\left(1 \mathrm{H}\right.$, ddddd, $\left.J=6.3 \mathrm{~Hz}, J=6.1 \mathrm{~Hz}, J=3.9 \mathrm{~Hz},{ }^{3} J_{\mathrm{HP}}=3.6 \mathrm{~Hz}, J=2.5 \mathrm{~Hz}, \mathrm{CHO}\right), 3.79(3 \mathrm{H}$, 
$\left.\mathrm{d},{ }^{3} J_{\mathrm{HP}}=11.0 \mathrm{~Hz}, \mathrm{P}(\mathrm{O}) \mathrm{OCH}_{3}\right), 3.80\left(3 \mathrm{H}, \mathrm{d},{ }^{3} J_{\mathrm{HP}}=11.0 \mathrm{~Hz}, \mathrm{P}(\mathrm{O}) \mathrm{OCH}_{3}\right) .{ }^{\mathbf{1 3}} \mathbf{C}-\mathbf{N M R}(\mathbf{7 5} \mathbf{~ M H z}$,

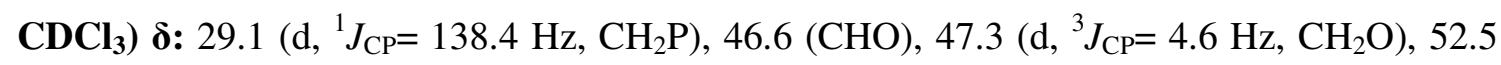
$\left(\mathrm{d},{ }^{2} J_{\mathrm{CP}}=4.6 \mathrm{~Hz}, 2 \times \mathrm{P}(\mathrm{O}) \mathrm{OCH}_{3}\right) .{ }^{31} \mathbf{P}-\mathbf{N M R}\left(\mathbf{1 2 1} \mathbf{M H z}, \mathbf{C D C l}_{3}\right) \boldsymbol{\delta}: 29.57 . \mathbf{I R}\left(\mathbf{c m}^{-\mathbf{1}}\right) \mathbf{v}_{\text {max }}$ : 1248 (P=O), 1018 (P-O). MS m/z (\%): (ES, Pos) 167 (M+H $\left.{ }^{+}, 100\right)$. Bp.: $55^{\circ} \mathrm{C}(0.5 \mathrm{~mm} \mathrm{Hg})$.

Elem. Anal. Calcd for $\mathrm{C}_{5} \mathrm{H}_{11} \mathrm{O}_{4} \mathrm{P}: \mathrm{C} 36.15$, H 6.67. Found: C 35.91, H 6.84. Yield: $80 \%$.

Synthesis of di(m)ethyl (1E)-3-oxoprop-1-enylphosphonate 4a,b (see Just, G.; Potvin, P.; Hakimelahi, G. H. Can. J. Chem. 1980, 58, 2780 for the preparation and spectral data of diethyl (1E)-3-hydroxyprop-1-enylphosphonate and diethyl (1E)-3-oxoprop-1enylphosphonate 4b)

Sodium methoxide in methanol (0.1 equiv) was added dropwise to di(m)ethyl 2,3epoxypropylphosphonate $\mathbf{3 a , b}(50 \mathrm{mmol})$ at $0{ }^{\circ} \mathrm{C}$ under stirring. After the addition, the reaction was stirred $0.5 \mathrm{~h}$ at room temperature. Then $2 \mathrm{~g}$ of Dowex 50W-X8 $\left(\mathrm{H}^{+}\right)$resin, previously washed with methanol, was added and the mixture was stirred for 15 min. Afterwards the resin was filtered off, washed with methanol, and the combined filtrate was evaporated to dryness to give the crude allylic alcohol, suitable for the next step.

Di(m)ethyl (1E)-3-hydroxyprop-1-enylphosphonate $(10 \mathrm{mmol})$ in $5 \mathrm{~mL}$ dry $\mathrm{CH}_{2} \mathrm{Cl}_{2}$ was added dropwise to a stirred mixture of $12 \mathrm{mmol}$ pyridinium chlorochromate and $3 \mathrm{~g}$ Celite in $30 \mathrm{~mL}$ dry $\mathrm{CH}_{2} \mathrm{Cl}_{2}$. The mixture was stirred for $2 \mathrm{~h}$ at room temperature and afterwards it was filtered through Celite. The Celite pad was washed several times with $\mathrm{CH}_{2} \mathrm{Cl}_{2}$, and the combined filtrate and washings were evaporated to dryness. The residue was triturated with ether and the solution was filtered through a Florisil pad, which was then washed several times with ether. The combined ethereal solutions were evaporated to dryness. After distillation in a Kugelrohr apparatus di(m)ethyl (1E)-3-oxoprop-1-enylphosphonate 4a,b was obtained as a yellowish/greenish oil.

\section{Dimethyl (1E)-3-oxoprop-1-enylphosphonate 4a}

${ }^{1} \mathbf{H}-\mathrm{NMR}\left(300 \mathrm{MHz}, \mathbf{C D C l}_{3}\right) \boldsymbol{\delta}: 3.82\left(6 \mathrm{H}, \mathrm{d},{ }^{3} J_{\mathrm{HP}}=11.0 \mathrm{~Hz}, 2 \times \mathrm{P}(\mathrm{O}) \mathrm{OCH}_{3}\right), 6.75(1 \mathrm{H}, \mathrm{dd}, J=$ $\left.17.3 \mathrm{~Hz},{ }^{2} J_{\mathrm{HP}}=16.8 \mathrm{~Hz}, \mathrm{CHP}\right), 6.87\left(1 \mathrm{H}, \mathrm{ddd},{ }^{3} J_{\mathrm{HP}}=19.8 \mathrm{~Hz}, J=17.3 \mathrm{~Hz}, J=6.9 \mathrm{~Hz}\right.$, $\underline{\mathrm{HC}}=\mathrm{CHP}), 9.70(1 \mathrm{H}, \mathrm{d}, J=6.9 \mathrm{~Hz}, \mathrm{HC}=\mathrm{O}) .{ }^{\mathbf{1 3}} \mathbf{C}-\mathbf{N M R}\left(\mathbf{7 5} \mathbf{M H z}, \mathbf{C D C l}_{3}\right) \boldsymbol{\delta}: 53.1\left(\mathrm{~d},{ }^{2} J_{\mathrm{CP}}=6.9\right.$ $\left.\mathrm{Hz}, 2 \times \mathrm{P}(\mathrm{O}) \mathrm{OCH}_{3}\right), 136.5\left(\mathrm{~d},{ }^{1} J_{\mathrm{CP}}=185.8 \mathrm{~Hz}, \mathrm{CHP}\right), 144.5\left(\mathrm{~d},{ }^{2} J_{\mathrm{CP}}=4.6 \mathrm{~Hz}, \mathrm{HC}=\mathrm{CHP}\right)$, $191.9\left(\mathrm{~d},{ }^{3} J_{\mathrm{CP}}=30.0 \mathrm{~Hz}, \mathrm{HC}=\mathrm{O}\right) .{ }^{31} \mathbf{P}-\mathbf{N M R}\left(\mathbf{1 2 1} \mathbf{M H z}, \mathbf{C D C l}_{\mathbf{3}}\right) \boldsymbol{\delta}: 17.09 . \mathbf{I R}\left(\mathbf{c m}^{\mathbf{- 1}}\right) \mathbf{v}_{\max }$ : $1701(\mathrm{C}=\mathrm{O}), 1257(\mathrm{P}=\mathrm{O}), 1050$ (br, P-O). MS m/z (\%): (ES, Pos) $165\left(\mathrm{M}+\mathrm{H}^{+}, 100\right)$. Bp.: 126 
${ }^{\circ} \mathrm{C}$ (4.5 mm Hg). Elem. Anal. Calcd for $\mathrm{C}_{5} \mathrm{H}_{9} \mathrm{O}_{4} \mathrm{P}: \mathrm{C}$ 36.60, H 5.53. Found: C 36.75, H 5.66.

Yield: $50 \%$.

Synthesis of di(m)ethyl (1E,3E)-3-(dimethylhydrazono)prop-1-enylphosphonate 6a,b

\section{Diethyl (1E,3E)-3-(dimethylhydrazono)prop-1-enylphosphonate 6b}

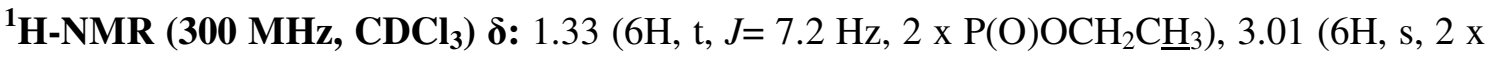

$\left.\mathrm{CH}_{3}\right), 4.08\left(4 \mathrm{H}, \mathrm{dq}, J=7.2 \mathrm{~Hz},{ }^{3} J_{\mathrm{HP}}=7.2 \mathrm{~Hz}, 2 \times \mathrm{P}(\mathrm{O}) \mathrm{OC}_{2} \mathrm{CH}_{3}\right), 5.62\left(1 \mathrm{H}, \mathrm{dd},{ }^{2} J_{\mathrm{HP}}=18.4\right.$ $\mathrm{Hz}, J=17.1 \mathrm{~Hz}, \mathrm{CHP}), 6.85(1 \mathrm{H}, \mathrm{d}, J=9.1 \mathrm{~Hz}, \mathrm{HC}=\mathrm{N}), 7.16\left(1 \mathrm{H}, \mathrm{ddd},{ }^{3} J_{\mathrm{HP}}=20.9 \mathrm{~Hz}, J=17.1\right.$ $\mathrm{Hz}, J=9.1 \mathrm{~Hz}, \underline{\mathrm{HC}}=\mathrm{CHP}) .{ }^{13} \mathbf{C}-\mathbf{N M R}\left(\mathbf{7 5} \mathbf{M H z}, \mathbf{C D C l}_{3}\right) \boldsymbol{\delta}: 16.4\left(\mathrm{~d},{ }^{3} J_{\mathrm{CP}}=5.8 \mathrm{~Hz}, 2 \mathrm{x}\right.$ $\left.\mathrm{P}(\mathrm{O}) \mathrm{OCH}_{2} \mathrm{CH}_{3}\right), 42.3\left(2 \times \mathrm{CH}_{3}\right), 61.66\left(\mathrm{~d},{ }^{2} J_{\mathrm{CP}}=5.8 \mathrm{~Hz}, 2 \times \mathrm{P}(\mathrm{O}) \mathrm{OCH}_{2} \mathrm{CH}_{3}\right), 61.70$ $\left(\mathrm{P}(\mathrm{O}) \mathrm{OCH}_{2} \mathrm{CH}_{3}\right), 112.7\left(\mathrm{~d},{ }^{1} J_{\mathrm{CP}}=196.1 \mathrm{~Hz}, \mathrm{CHP}\right), 129.6\left(\mathrm{~d},{ }^{3} J_{\mathrm{CP}}=31.2 \mathrm{~Hz}, \mathrm{HC}=\mathrm{N}\right), 146.9(\mathrm{~d}$, $\left.{ }^{2} J_{\mathrm{CP}}=6.9 \mathrm{~Hz}, \mathrm{HC}=\mathrm{CHP}\right) .{ }^{31} \mathbf{P}-\mathbf{N M R}\left(\mathbf{1 2 1} \mathbf{M H z}, \mathbf{C D C l}_{\mathbf{3}}\right) \boldsymbol{\delta}: 20.84 . \mathbf{I R}\left(\mathbf{c m}^{-\mathbf{1}}\right) \mathbf{v}_{\max }: 1598$ $(\mathrm{C}=\mathrm{N}), 1240$ (P=O), 1047 (P-O), 1027 (P-O). MS m/z (\%): (ES, Pos) 235 (M+H', 40). Elem. Anal. Calcd for $\mathrm{C}_{9} \mathrm{H}_{19} \mathrm{~N}_{2} \mathrm{O}_{3} \mathrm{P}$ : C 46.15, H 8.18, N 11.96. Found: C 46.33, H 8.20, N 12.24. Yield: $82 \%$.

\section{Synthesis of $\gamma$-phosphono- $\alpha$-aminobisphosphonates 9}

General procedure for the preparation of DAPTMS: dialkyl phosphite $(30 \mathrm{mmol})$ was mixed with $33 \mathrm{mmol}$ of triethylamine (1,1 equiv) in $40 \mathrm{~mL}$ of dry dichloromethane in an oven dry flask under a nitrogen atmosphere. The mixture was then cooled to $0{ }^{\circ} \mathrm{C}$ and $33 \mathrm{mmol}$ of TMSCl (1.1 equiv) was added using a syringe. After $1 \mathrm{~h}$ at $0^{\circ} \mathrm{C}$, the DAP was completely converted to the DAPTMS (this could easily be monitored using ${ }^{31} \mathrm{P}$ NMR (DAP: $\delta=5-15$ ppm; DAPTMS: $\delta=120-130 \mathrm{ppm}$ ). The triethylammonium chloride salts were removed by filtration (care had to be taken to avoid contact with moisture) and the dichloromethane was evaporated under reduced pressure. Then, $20 \mathrm{~mL}$ of dry diethyl ether was added to the residue in order to precipitate the remaining triethylammonium chloride from the mixture. After filtration and evaporation of the solvent, the DAPTMS was obtained as a clear, colourless liquid and could be stored for several weeks at $-20{ }^{\circ} \mathrm{C}$ when kept away from moisture.

\section{Dimethyl [1,3-bis(dimethoxyphosphoryl)-1-sec-butylaminopropyl]phosphonate 9b}

${ }^{1} \mathbf{H}-\mathrm{NMR}\left(300 \mathrm{MHz}, \mathbf{C D C l}_{3}\right) \boldsymbol{\delta}: 0.87\left(3 \mathrm{H}, \mathrm{t}, J=7.4 \mathrm{~Hz}, \mathrm{CH}_{2} \mathrm{CH}_{3}, \mathrm{sec}-\mathrm{Bu}\right), 1.08(3 \mathrm{H}, \mathrm{d}, J=6.3$ $\mathrm{Hz}, \mathrm{CH}_{3}$, sec- $\left.\mathrm{Bu}\right), 1.22-1.40\left(1 \mathrm{H}, \mathrm{m}, \mathrm{C}_{\mathrm{H}} \mathrm{H}_{\mathrm{B}} \mathrm{CH}_{3}\right.$, sec- $\left.\mathrm{Bu}\right), 1.47-1.60\left(1 \mathrm{H}, \mathrm{m}, \mathrm{CH}_{\mathrm{A}} \underline{\mathrm{H}}_{\mathrm{B}} \mathrm{CH}_{3}\right.$, sec-Bu), $1.79(1 \mathrm{H}$, br s, $\mathrm{NH}), 2.09-2.35\left(4 \mathrm{H}, \mathrm{m}, \mathrm{C}_{2} \mathrm{C}_{2} \mathrm{P}\right), 3.02(1 \mathrm{H}, \mathrm{m}, \mathrm{NCH}$, sec-Bu $), 3.75$ 
$\left(6 \mathrm{H}, \mathrm{d},{ }^{3} J_{\mathrm{HP}}=10.7 \mathrm{~Hz}, 2 \times \mathrm{P}(\mathrm{O})\left(\mathrm{OCH}_{3}\right)\right), 3.84\left(6 \mathrm{H}, \mathrm{d},{ }^{3} J_{\mathrm{HP}}=10.7 \mathrm{~Hz}, 2 \times \mathrm{P}(\mathrm{O})\left(\mathrm{OCH}_{3}\right)\right), 3.86$ $\left(6 \mathrm{H}, \mathrm{d},{ }^{3} J_{\mathrm{HP}}=10.7 \mathrm{~Hz}, 2 \times \mathrm{P}(\mathrm{O})\left(\mathrm{OCH}_{3}\right)\right) .{ }^{\mathbf{1 3}} \mathbf{C}-\mathbf{N M R}\left(\mathbf{7 5} \mathbf{M H z}, \mathbf{C D C l}_{3}\right) \boldsymbol{\delta}: 10.2\left(\mathrm{CH}_{2} \mathrm{CH}_{3}\right.$, sec$\mathrm{Bu}), 20.1\left(\mathrm{dt},{ }^{1} J_{\mathrm{CP}}=140.8 \mathrm{~Hz},{ }^{3} J_{\mathrm{CP}}=5.2 \mathrm{~Hz}, \mathrm{CH}_{2} \mathrm{P}\right), 22.3\left(\mathrm{CH}_{3}, \sec -\mathrm{Bu}\right), 24.4\left(\underline{\mathrm{CH}}_{2} \mathrm{CH}_{2} \mathrm{P}\right)$, $32.3\left(\mathrm{CH}_{2} \mathrm{CH}_{3}\right.$, sec-Bu), $49.4\left(\mathrm{t},{ }^{3} J_{\mathrm{CP}}=6.9 \mathrm{~Hz}, \mathrm{NCH}\right.$, sec-Bu $), 52.4\left(\mathrm{~d},{ }^{2} J_{\mathrm{CP}}=6.9 \mathrm{~Hz}, 2 \mathrm{x}\right.$ $\left.\mathrm{P}(\mathrm{O})\left(\mathrm{OCH}_{3}\right)\right), 54.0\left(\mathrm{dt},{ }^{2} J_{\mathrm{CP}}=38.1 \mathrm{~Hz},{ }^{4} J_{\mathrm{CP}}=4.6 \mathrm{~Hz}, 4 \mathrm{x} \mathrm{P}(\mathrm{O}) \mathrm{OCH}_{3}\right), 62.4\left(\mathrm{dt},{ }^{1} J_{\mathrm{CP}}=140.8 \mathrm{~Hz}\right.$, $\left.{ }^{3} J_{\mathrm{CP}}=19.6 \mathrm{~Hz}, \mathrm{C}_{\text {quat }} \mathrm{P}_{2}\right) .{ }^{31} \mathbf{P}-\mathbf{N M R}\left(\mathbf{1 2 1} \mathbf{M H z}, \mathbf{C D C l}_{\mathbf{3}}\right) \boldsymbol{\delta}: 24.53\left(2 \times \mathrm{P}(\mathrm{O})\left(\mathrm{OCH}_{3}\right)_{2}\right), 34.73$

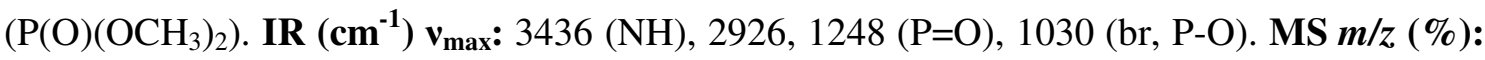
(ES, Pos) $440\left(\mathrm{M}+\mathrm{H}^{+}, 100\right), 330\left(\mathrm{M}^{+}-\mathrm{P}(\mathrm{O})\left(\mathrm{OCH}_{3}\right)_{2}\right.$, 84). Elem. Anal. Calcd for $\mathrm{C}_{13} \mathrm{H}_{32} \mathrm{NO}_{9} \mathrm{P}_{3}$ : C 35.54, H 7.34, N 3.19. Found: C 35.53, H 7.05, N 3.01. Yield: $77 \%$.

\section{Dimethyl [1,3-bis(dimethoxyphosphoryl)-1-isobutylaminopropyl]phosphonate 9c}

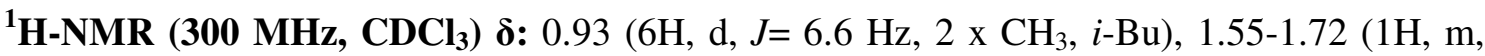
$\mathrm{CH}, i-\mathrm{Bu}), 1.76(1 \mathrm{H}$, br s, $\mathrm{NH}), 2.01-2.35\left(4 \mathrm{H}, \mathrm{m}, \mathrm{C}_{2} \mathrm{C}_{2} \mathrm{P}\right), 2.51\left(2 \mathrm{H}, \mathrm{d}, J=6.3 \mathrm{~Hz}, \mathrm{NCH}_{2}\right.$, $i$-Bu), $3.76\left(6 \mathrm{H}, \mathrm{d},{ }^{3} J_{\mathrm{HP}}=10.7 \mathrm{~Hz}, 2 \times \mathrm{P}(\mathrm{O}) \mathrm{OMe}\right), 3.84\left(6 \mathrm{H}, \mathrm{d},{ }^{3} J_{\mathrm{HP}}=10.7 \mathrm{~Hz}, 2 \times \mathrm{P}(\mathrm{O}) \mathrm{OMe}\right)$, $3.86\left(6 \mathrm{H}, \mathrm{d},{ }^{3} J_{\mathrm{HP}}=10.7 \mathrm{~Hz}, 2 \times \mathrm{P}(\mathrm{O}) \mathrm{OMe}\right) .{ }^{13} \mathbf{C}-\mathbf{N M R}\left(75 \mathbf{M H z}, \mathbf{C D C l}_{\mathbf{3}}\right) \boldsymbol{\delta}: 19.2\left(\mathrm{dt},{ }^{1} J_{\mathrm{CP}}=\right.$ $\left.140.8 \mathrm{~Hz},{ }^{3} J_{\mathrm{CP}}=5.8 \mathrm{~Hz}, \mathrm{CH}_{2} \mathrm{P}\right), 20.7\left(2 \times \mathrm{CH}_{3}, i-\mathrm{Bu}\right), 22.8\left(\mathrm{CH}_{2} \mathrm{CH}_{2} \mathrm{P}\right), 29.4(\mathrm{CH}, i-\mathrm{Bu}), 50.9$ $\left(\mathrm{t},{ }^{3} J_{\mathrm{CP}}=5.8 \mathrm{~Hz}, \mathrm{NCH}_{2}, i-\mathrm{Bu}\right), 52.4\left(\mathrm{~d},{ }^{2} J_{\mathrm{CP}}=5.8 \mathrm{~Hz}, 2 \times \mathrm{P}(\mathrm{O}) \mathrm{OMe}\right), 53.9\left(\mathrm{dt},{ }^{2} J_{\mathrm{CP}}=42.7 \mathrm{~Hz}\right.$, $\left.{ }^{4} J_{\mathrm{CP}}=3.5 \mathrm{~Hz}, 4 \times \mathrm{P}(\mathrm{O}) \mathrm{OMe}\right), 62.4\left(\mathrm{dt},{ }^{1} J_{\mathrm{CP}}=140.8 \mathrm{~Hz},{ }^{3} J_{\mathrm{CP}}=18.5 \mathrm{~Hz}, \mathrm{C}_{\text {quat }} \mathrm{P}_{2}\right) .{ }^{31} \mathbf{P}-\mathbf{N M R}(\mathbf{1 2 1}$

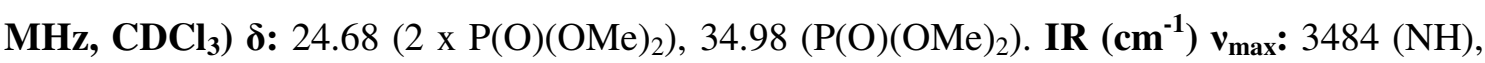
2958, 1247 (P=O), 1030 (br, P-O). MS m/z (\%): (ES, Pos) $440\left(\mathrm{M}+\mathrm{H}^{+}, 100\right), 330\left(\mathrm{M}^{+}-\right.$ $\mathrm{P}(\mathrm{O})(\mathrm{OMe})_{2}$, 14). Elem. Anal. Calcd for $\mathrm{C}_{13} \mathrm{H}_{32} \mathrm{NO}_{9} \mathrm{P}_{3}$ : C 35.54, H 7.34, N 3.19. Found: C 35.70, H 7.37, N 3.13. Yield: $72 \%$.

\section{Dimethyl [1,3-bis(dimethoxyphosphoryl)-1-cyclohexylaminopropyl]phosphonate 9d}

${ }^{1}$ H-NMR (300 MHz, $\mathbf{C D C l}_{3}$ ) $\boldsymbol{\delta}$ : 1.00-1.94 (11H, m, 5 x CH $\left.2, c-H e x+\mathrm{NH}\right), 2.08-2.34(4 \mathrm{H}$, m, $\left.\underline{\mathrm{C}}_{2} \underline{\mathrm{C}}_{2} \mathrm{P}\right), 2.77-2.88(1 \mathrm{H}, \mathrm{m}, \mathrm{NCH}, c-\mathrm{Hex}), 3.75\left(6 \mathrm{H}, \mathrm{d},{ }^{3} J_{\mathrm{HP}}=11.0 \mathrm{~Hz}, 2 \times \mathrm{P}(\mathrm{O}) \mathrm{OCH}_{3}\right)$, $3.84\left(6 \mathrm{H}, \mathrm{d},{ }^{3} J_{\mathrm{HP}}=11.0 \mathrm{~Hz}, 2 \times \mathrm{P}(\mathrm{O}) \mathrm{OCH}_{3}\right), 3.86\left(6 \mathrm{H}, \mathrm{d},{ }^{3} J_{\mathrm{HP}}=11.0 \mathrm{~Hz}, 2 \times \mathrm{P}(\mathrm{O}) \mathrm{OCH}_{3}\right) .{ }^{13} \mathrm{C}-$ NMR (75 MHz, $\left.\mathbf{C D C l}_{3}\right) \boldsymbol{\delta}: 20.0\left(\mathrm{dt},{ }^{1} J_{\mathrm{CP}}=140.8 \mathrm{~Hz},{ }^{3} J_{\mathrm{CP}}=5.2 \mathrm{~Hz}, \mathrm{CH}_{2} \mathrm{P}\right), 24.3\left(\underline{C H}_{2} \mathrm{CH}_{2} \mathrm{P}\right)$, $25.5\left(2 \mathrm{x} \mathrm{CH}_{2}, c\right.$-Hex), $25.6\left(\mathrm{CH}_{2}, c-\mathrm{Hex}\right), 36.4\left(2 \mathrm{x} \mathrm{CH}_{2}, c-\mathrm{Hex}\right), 51.6\left(\mathrm{t},{ }^{3} J_{\mathrm{CP}}=6.3 \mathrm{~Hz}, \mathrm{NCH}\right.$, $c$-Hex), $52.4\left(\mathrm{~d},{ }^{2} J_{\mathrm{CP}}=5.8 \mathrm{~Hz}, 2 \times \mathrm{P}(\mathrm{O}) \mathrm{OCH}_{3}\right), 54.0\left(\mathrm{dt},{ }^{2} J_{\mathrm{CP}}=47.3 \mathrm{~Hz},{ }^{4} J_{\mathrm{CP}}=6.9 \mathrm{~Hz}, 4 \mathrm{x}\right.$ $\left.\mathrm{P}(\mathrm{O}) \mathrm{OCH}_{3}\right), 62.7\left(\mathrm{dt},{ }^{1} J_{\mathrm{CP}}=140.8 \mathrm{~Hz},{ }^{3} J_{\mathrm{CP}}=18.5 \mathrm{~Hz}, \mathrm{C}_{\mathrm{quat}} \mathrm{P}_{2}\right) .{ }^{31} \mathbf{P}-\mathbf{N M R}\left(\mathbf{1 2 1} \mathbf{M H z}, \mathbf{C D C l}_{3}\right)$

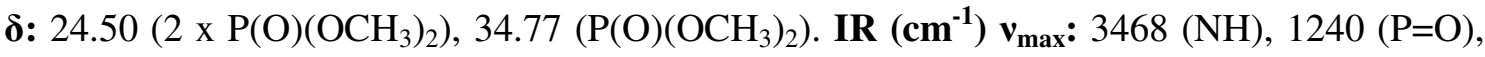
1029 (br, P-O). MS $\boldsymbol{m} / z$ (\%): (ES, Pos) $466\left(\mathrm{M}+\mathrm{H}^{+}, 100\right), 356\left(\mathrm{M}^{+}-\mathrm{P}(\mathrm{O})\left(\mathrm{OCH}_{3}\right)_{2}, 55\right)$. Elem. 
Anal. Calcd for $\mathrm{C}_{15} \mathrm{H}_{34} \mathrm{NO}_{9} \mathrm{P}_{3}$ : C 38.71, H 7.36, N 3.01. Found: C 38.73, H 7.47, N 2.95. Yield: $79 \%$.

\section{Dimethyl [1,3-bis(dimethoxyphosphoryl)-1-benzylaminopropyl]phosphonate 9e}

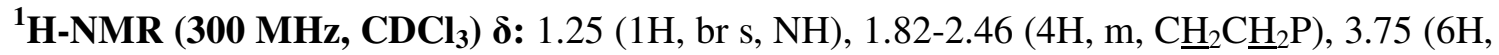
$\left.\mathrm{d},{ }^{3} J_{\mathrm{HP}}=11.0 \mathrm{~Hz}, 2 \times \mathrm{P}(\mathrm{O})\left(\mathrm{OCH}_{3}\right)\right), 3.88\left(12 \mathrm{H}, \mathrm{d},{ }^{3} J_{\mathrm{HP}}=11.0 \mathrm{~Hz}, 4 \times \mathrm{P}(\mathrm{O})\left(\mathrm{OCH}_{3}\right)\right), 3.91(2 \mathrm{H}$,

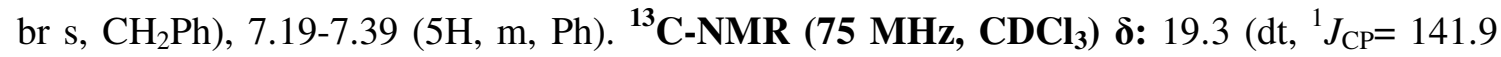
$\left.\mathrm{Hz},{ }^{3} J_{\mathrm{CP}}=5.8 \mathrm{~Hz}, \mathrm{CH}_{2} \mathrm{P}\right), 23.4\left(\mathrm{CH}_{2} \mathrm{CH}_{2} \mathrm{P}\right), 47.9\left(\mathrm{t},{ }^{3} J_{\mathrm{CP}}=6.4 \mathrm{~Hz}, \mathrm{NCH}_{2}\right), 52.5\left(\mathrm{~d},{ }^{2} J_{\mathrm{CP}}=5.8\right.$ $\left.\mathrm{Hz}, 2 \times \mathrm{P}(\mathrm{O}) \mathrm{OCH}_{3}\right), 54.1\left(\mathrm{dt},{ }^{2} J_{\mathrm{CP}}=38.1 \mathrm{~Hz},{ }^{4} J_{\mathrm{CP}}=3.5 \mathrm{~Hz}, 4 \times \mathrm{P}(\mathrm{O}) \mathrm{OCH}_{3}\right), 62.5\left(\mathrm{dt},{ }^{1} J_{\mathrm{CP}}=\right.$ $\left.140.8 \mathrm{~Hz},{ }^{3} J_{\mathrm{CP}}=18.5 \mathrm{~Hz}, \mathrm{C}_{\mathrm{quat}} \mathrm{P}_{2}\right), 127.4\left(\mathrm{CH}_{\text {arom }}\right), 128.3\left(2 \times \mathrm{CH}_{\text {arom }}\right), 128.6\left(2 \times \mathrm{CH}_{\text {arom }}\right)$, 139.7 ( $\left.\mathrm{C}_{\text {quat,arom }}\right) .{ }^{31} \mathbf{P}-\mathbf{N M R}\left(\mathbf{1 2 1} \mathbf{M H z}, \mathbf{C D C l}_{\mathbf{3}}\right) \quad \boldsymbol{\delta}: 24.35\left(2 \mathrm{x} \mathrm{P}(\mathrm{O})\left(\mathrm{OCH}_{3}\right)_{2}\right), 34.76$

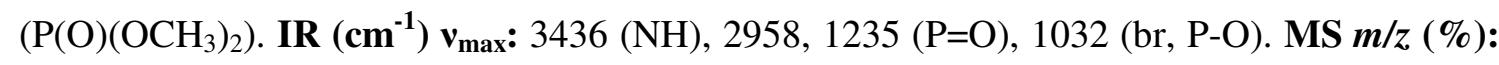
(ES, Pos) $474\left(\mathrm{M}+\mathrm{H}^{+}, 100\right), 364\left(\mathrm{M}^{+}-\mathrm{P}(\mathrm{O})(\mathrm{OMe})_{2}, 12\right), 276$ (57). Elem. Anal. Calcd for $\mathrm{C}_{16} \mathrm{H}_{30} \mathrm{NO}_{9} \mathrm{P}_{3}$ : C 40.60, H 6.39, N 2.96. Found: C 40.57, H 6.42, N 3.21. Yield: $44 \%$.

\section{Diethyl [1,3-bis(diethoxyphosphoryl)-1-isopropylaminopropyl]phosphonate 9f}

${ }^{1} \mathbf{H}-\mathbf{N M R}\left(300 \mathrm{MHz}, \mathbf{C D C l}_{3}\right) \boldsymbol{\delta}: 1.10\left(6 \mathrm{H}, \mathrm{d}, J=6.3 \mathrm{~Hz}, 2 \times \mathrm{CH}_{3}, i\right.$-Pr), $1.33(6 \mathrm{H}, \mathrm{t}, J=7.0$ $\left.\mathrm{Hz}, 2 \times \mathrm{P}(\mathrm{O}) \mathrm{OCH}_{2} \mathrm{CH}_{3}\right), 1.34\left(12 \mathrm{H}, \mathrm{t}, J=7.0 \mathrm{~Hz}, 2 \times \mathrm{P}(\mathrm{O}) \mathrm{OCH}_{2} \mathrm{CH}_{3}\right), 1.58$ (1H, br s, NH), 2.12-2.36 (4H, m, $\left.\underline{\mathrm{C}}_{2} \mathrm{C}_{2} \mathrm{P}\right), 3.32$ (1H, sept, $\left.J=6.3 \mathrm{~Hz}, \mathrm{NCH}, i-\mathrm{Pr}\right), 4.04-4.28$ (12H, m, $6 \mathrm{x}$ $\left.\mathrm{P}(\mathrm{O}) \mathrm{OC}_{2} \mathrm{CH}_{3}\right) .{ }^{13} \mathbf{C}-\mathrm{NMR}\left(75 \mathrm{MHz}, \mathbf{C D C l}_{3}\right) \boldsymbol{\delta}: 16.5\left(\mathrm{~d},{ }^{3} J_{\mathrm{CP}}=5.8 \mathrm{~Hz}, 6\right.$ x P(O)OCH$\left.\underline{\mathrm{CH}}_{3}\right)$, $21.1\left(\mathrm{dt},{ }^{1} J_{\mathrm{CP}}=140.8 \mathrm{~Hz},{ }^{3} J_{\mathrm{CP}}=4.6 \mathrm{~Hz}, \mathrm{CH}_{2} \mathrm{P}\right), 24.6\left(\mathrm{CH}_{2} \mathrm{CH}_{2} \mathrm{P}\right), 26.0\left(2 \mathrm{x} \mathrm{CH}_{3}, i-\mathrm{Pr}\right), 44.1$ (t, $\left.{ }^{3} J_{\mathrm{CP}}=6.9 \mathrm{~Hz}, \mathrm{NCH}, i-\mathrm{Pr}\right), 61.5\left(\mathrm{~d},{ }^{2} J_{\mathrm{CP}}=5.8 \mathrm{~Hz}, 2 \times \mathrm{P}(\mathrm{O}) \mathrm{OCH}_{2} \mathrm{CH}_{3}\right), 62.4\left(\mathrm{dt},{ }^{1} J_{\mathrm{CP}}=140.8\right.$ $\left.\mathrm{Hz},{ }^{3} J_{\mathrm{CP}}=19.6 \mathrm{~Hz}, \mathrm{C}_{\mathrm{quat}} \mathrm{P}_{2}\right), 63.2\left(\mathrm{dt},{ }^{2} J_{\mathrm{CP}}=38.1 \mathrm{~Hz},{ }^{4} J_{\mathrm{CP}}=3.5 \mathrm{~Hz}, 4\right.$ x P(O)OCH $\left.\mathrm{H}_{2} \mathrm{CH} 3\right) .{ }^{31} \mathbf{P}-$

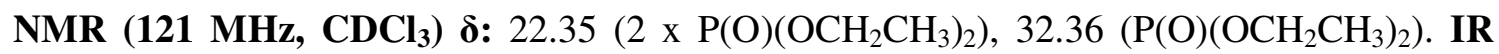
$\left(\mathbf{c m}^{-1}\right) v_{\text {max }}: 3470(\mathrm{NH}), 2981,1237(\mathrm{P}=\mathrm{O}), 1027$ (br, P-O). MS $\boldsymbol{m} / \boldsymbol{z}(\boldsymbol{\%})$ : (ES, Pos) 510 $\left(\mathrm{M}+\mathrm{H}^{+}, 100\right), 372\left(\mathrm{M}^{+}-\mathrm{P}(\mathrm{O})(\mathrm{OEt})_{2}, 48\right)$. Elem. Anal. Calcd for $\mathrm{C}_{18} \mathrm{H}_{42} \mathrm{NO}_{9} \mathrm{P}_{3}: \mathrm{C} 42.44, \mathrm{H}$ 8.31, N 2.75. Found: C 42.26, H 8.50, N 2.81. Yield: $76 \%$.

Diethyl [1,3-bis(diethoxyphosphoryl)-1-sec-butylaminopropyl]phosphonate 9g ${ }^{1} \mathbf{H}-N M R\left(300 ~ M H z, \mathbf{C D C l}_{3}\right) \boldsymbol{\delta}: 0.86\left(3 \mathrm{H}, \mathrm{t}, J=7.4 \mathrm{~Hz}, \mathrm{CH}_{2} \mathrm{CH}_{3}, \mathrm{sec}-\mathrm{Bu}\right), 1.08(3 \mathrm{H}, \mathrm{d}, J=6.3$ $\mathrm{Hz}, \mathrm{CH}_{3}$, sec- $\left.\mathrm{Bu}\right), 1.33\left(6 \mathrm{H}, \mathrm{t}, J=7.0 \mathrm{~Hz}, 2 \times \mathrm{P}(\mathrm{O}) \mathrm{OCH}_{2} \underline{\mathrm{C}}_{3}\right), 1.34(12 \mathrm{H}, \mathrm{t}, J=7.0 \mathrm{~Hz}, 2 \mathrm{x}$ $\left.\mathrm{P}(\mathrm{O}) \mathrm{OCH}_{2} \mathrm{CH}_{3}\right), 1.48-1.62\left(2 \mathrm{H}, \mathrm{m}, \mathrm{C}_{2} \mathrm{CH}_{3}\right.$, sec-Bu $), 2.08$ (1H, br s, NH), 2.12-2.36 (4H, m, $\left.\mathrm{C}_{2} \mathrm{CH}_{2} \mathrm{P}\right), 3.03-3.11\left(1 \mathrm{H}, \mathrm{m}, \mathrm{NCH}\right.$, sec-Bu), 4.04-4.27 (12H, m, 6 x P(O)OC $\left.\underline{\mathrm{H}}_{2} \mathrm{CH}_{3}\right) .{ }^{13} \mathrm{C}-$

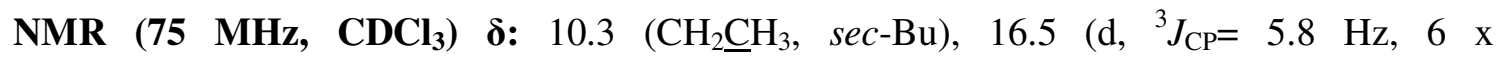


$\left.\mathrm{P}(\mathrm{O}) \mathrm{OCH}_{2} \mathrm{CH}_{3}\right), 21.2\left(\mathrm{dt},{ }^{1} J_{\mathrm{CP}}=139.6 \mathrm{~Hz},{ }^{3} J_{\mathrm{CP}}=4.6 \mathrm{~Hz}, \mathrm{CH}_{2} \mathrm{P}\right), 22.4\left(\mathrm{CH}_{3}\right.$, sec $\left.-\mathrm{Bu}\right), 24.7$ $\left(\underline{\mathrm{CH}}_{2} \mathrm{CH}_{2} \mathrm{P}\right), 32.3\left(\underline{\mathrm{CH}}_{2} \mathrm{CH}_{3}\right.$, sec-Bu $), 49.2\left(\mathrm{t},{ }^{3} J_{\mathrm{CP}}=6.9 \mathrm{~Hz}, \mathrm{NCH}, \sec -\mathrm{Bu}\right), 61.5\left(\mathrm{~d},{ }^{2} J_{\mathrm{CP}}=6.9\right.$ $\left.\mathrm{Hz}, 2 \times \mathrm{P}(\mathrm{O}) \mathrm{OCH}_{2} \mathrm{CH}_{3}\right), 62.3\left(\mathrm{dt},{ }^{1} J_{\mathrm{CP}}=140.8 \mathrm{~Hz},{ }^{3} J_{\mathrm{CP}}=19.6 \mathrm{~Hz}, \mathrm{C}_{\text {quat }} \mathrm{P}_{2}\right), 63.2\left(\mathrm{dt},{ }^{2} J_{\mathrm{CP}}=28.8\right.$ $\left.\mathrm{Hz},{ }^{4} J_{\mathrm{CP}}=3.5 \mathrm{~Hz}, 4 \mathrm{x} \mathrm{P}(\mathrm{O}) \mathrm{OCH}_{2} \mathrm{CH} 3\right) .{ }^{31} \mathbf{P}-\mathrm{NMR}\left(\mathbf{1 2 1} \mathbf{M H z}, \mathbf{C D C l}_{3}\right)$ 8: $22.38(2 \mathrm{x}$ $\left.\mathrm{P}(\mathrm{O})\left(\mathrm{OCH}_{2} \mathrm{CH}_{3}\right)_{2}\right), 32.38\left(\mathrm{P}(\mathrm{O})\left(\mathrm{OCH}_{2} \mathrm{CH}_{3}\right)_{2}\right)$. IR ( (cm $\left.^{-\mathbf{1}}\right) \mathbf{v}_{\text {max }}: 3470(\mathrm{NH}), 2979,2929,1246$ (P=O), 1028 (br, P-O). MS m/z (\%): (ES, Pos) $524\left(\mathrm{M}+\mathrm{H}^{+}, 100\right), 386\left(\mathrm{M}^{+}-\mathrm{P}(\mathrm{O})(\mathrm{OEt})_{2}, 32\right)$. Elem. Anal. Calcd for $\mathrm{C}_{19} \mathrm{H}_{44} \mathrm{NO}_{9} \mathrm{P}_{3}$ : C 43.59, H 8.47, N 2.68. Found: C 43.78, H 8.65, N 2.71. Yield: $72 \%$.

\section{Diethyl [1,3-bis(diethoxyphosphoryl)-1-cyclohexylaminopropyl]phosphonate 9h}

${ }^{1}$ H-NMR (300 MHz, $\left.\mathbf{C D C l}_{3}\right)$ d: 1.29-1.37 (18H, m, 6 x P(O)OCH$\left.{ }_{2} \underline{\mathrm{H}}_{3}\right), 1.02-1.92(11 \mathrm{H}, \mathrm{m}$, 5 x CH$\left.{ }_{2}, c-\mathrm{Hex}+\mathrm{NH}\right), 2.10-2.34\left(4 \mathrm{H}, \mathrm{m}, \mathrm{C}_{2} \mathrm{C}_{2} \mathrm{P}\right), 2.82-2.92(1 \mathrm{H}, \mathrm{m}, \mathrm{NCH}, c-\mathrm{Hex}), 4.04-$ $4.27\left(12 \mathrm{H}, \mathrm{m}, 6 \mathrm{x} \mathrm{P}(\mathrm{O}) \mathrm{OC} \underline{\mathrm{H}}_{2} \mathrm{CH}_{3}\right) .{ }^{13} \mathbf{C}-\mathrm{NMR}\left(75 \mathbf{M H z}, \mathbf{C D C l}_{3}\right) \boldsymbol{\delta}: 16.5\left(\mathrm{~d},{ }^{3} \mathrm{~J}_{\mathrm{CP}}=5.8 \mathrm{~Hz}, 6 \mathrm{x}\right.$ $\left.\mathrm{P}(\mathrm{O}) \mathrm{OCH}_{2} \underline{\mathrm{CH}}_{3}\right), 21.1\left(\mathrm{dt},{ }^{1} J_{\mathrm{CP}}=140.8 \mathrm{~Hz},{ }^{3} J_{\mathrm{CP}}=4.6 \mathrm{~Hz}, \mathrm{CH}_{2} \mathrm{P}\right), 24.6\left(\underline{\mathrm{CH}}_{2} \mathrm{CH}_{2} \mathrm{P}\right), 25.6(2 \mathrm{x}$ $\mathrm{CH}_{2}, c$-Hex), $25.7\left(\mathrm{CH}_{2}, c-\mathrm{Hex}\right), 36.5\left(2 \mathrm{x} \mathrm{CH}_{2}, c-\mathrm{Hex}\right), 51.4\left(\mathrm{t},{ }^{3} J_{\mathrm{CP}}=6.9 \mathrm{~Hz}, \mathrm{NCH}, c-\mathrm{Hex}\right)$, $61.5\left(\mathrm{~d},{ }^{2} J_{\mathrm{CP}}=5.8 \mathrm{~Hz}, 2 \times \mathrm{P}(\mathrm{O}) \mathrm{OCH}_{2} \mathrm{CH}_{3}\right), 62.2\left(\mathrm{dt},{ }^{1} J_{\mathrm{CP}}=140.8 \mathrm{~Hz},{ }^{3} J_{\mathrm{CP}}=19.6 \mathrm{~Hz}, \mathrm{C}_{\text {quat }} \mathrm{P}_{2}\right)$, $63.3\left(\mathrm{dt},{ }^{2} J_{\mathrm{CP}}=36.9 \mathrm{~Hz},{ }^{4} J_{\mathrm{CP}}=3.5 \mathrm{~Hz}, 4 \mathrm{x} \mathrm{P}(\mathrm{O}) \mathrm{OCH}_{2} \mathrm{CH} 3\right) .{ }^{31} \mathbf{P}-\mathbf{N M R}\left(\mathbf{1 2 1} \mathbf{M H z}, \mathbf{C D C l}_{3}\right) \boldsymbol{\delta}$ : $22.31\left(2 \times \mathrm{P}(\mathrm{O})\left(\mathrm{OCH}_{2} \mathrm{CH}_{3}\right)_{2}\right), 32.39\left(\mathrm{P}(\mathrm{O})\left(\mathrm{OCH}_{2} \mathrm{CH}_{3}\right)_{2}\right)$. IR ( $\left.\mathbf{c m}^{-\mathbf{1}}\right) \mathbf{v}_{\mathbf{m a x}}: 3480(\mathrm{NH}), 1246$ $(\mathrm{P}=\mathrm{O}), 1026$ (br, P-O). MS m/z (\%): (ES, Pos) $550\left(\mathrm{M}+\mathrm{H}^{+}, 100\right), 412\left(\mathrm{M}^{+}-\mathrm{P}(\mathrm{O})\left(\mathrm{OCH}_{2} \mathrm{CH}_{3}\right)_{2}\right.$, 40). Elem. Anal. Calcd for $\mathrm{C}_{21} \mathrm{H}_{46} \mathrm{NO}_{9} \mathrm{P}_{3}$ : C 45.90, H 8.44, N 2.55. Found: C 45.79, H 8.51, N 2.45. Yield: $74 \%$. 
Dimethyl (1E,3E)-3-(t-butylimino)prop-1-enylphosphonate 1a ( ${ }^{1} \mathrm{H}-\mathrm{NMR}$ : $\left.300 \mathrm{MHz}, \mathrm{CDCl}_{3}\right)$

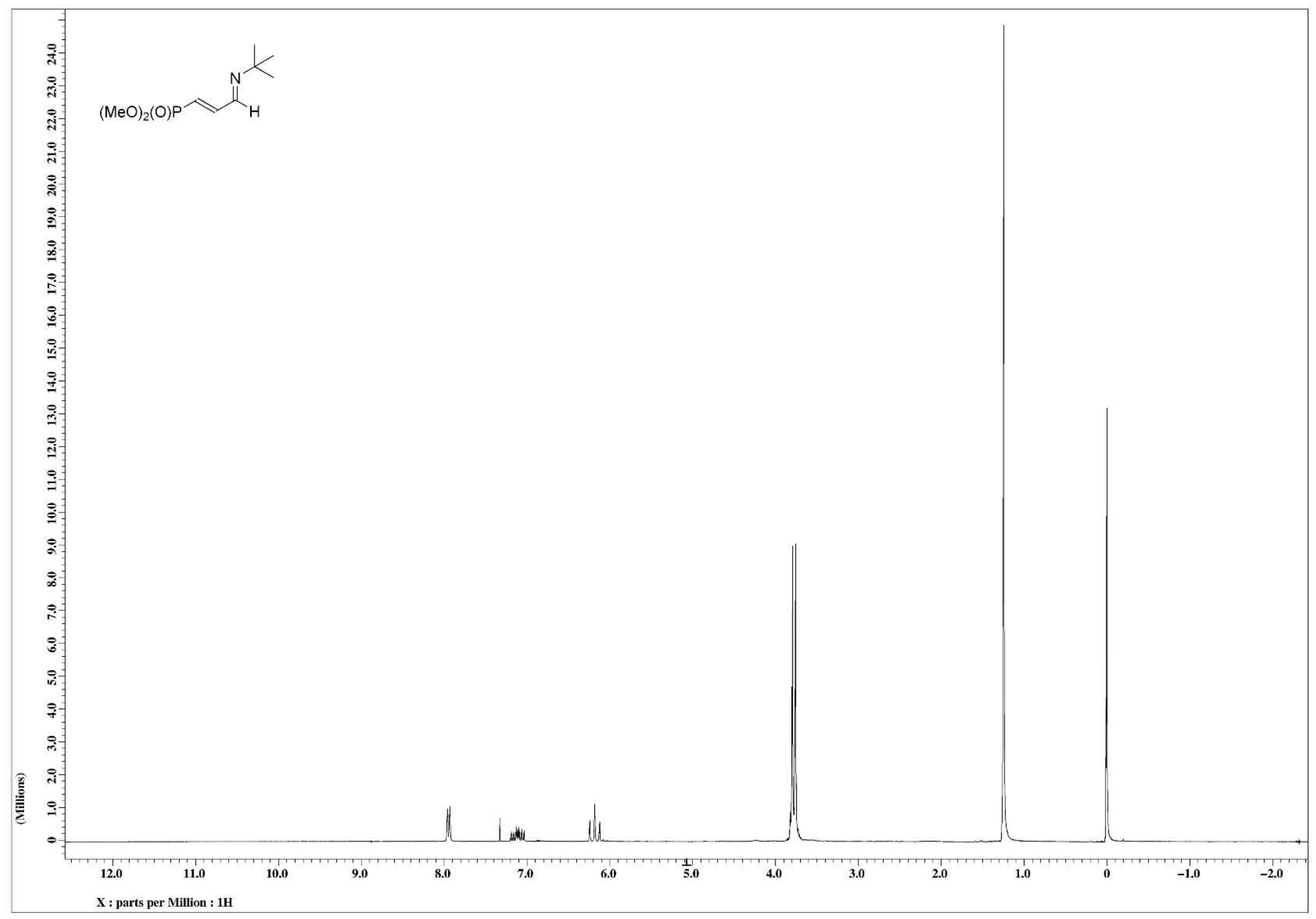

S 12 
Dimethyl (1E,3E)-3-(t-butylimino)prop-1-enylphosphonate 1a $\left({ }^{13} \mathrm{C}-\mathrm{NMR}\right.$ : $\left.75 \mathrm{MHz}, \mathrm{CDCl}_{3}\right)$

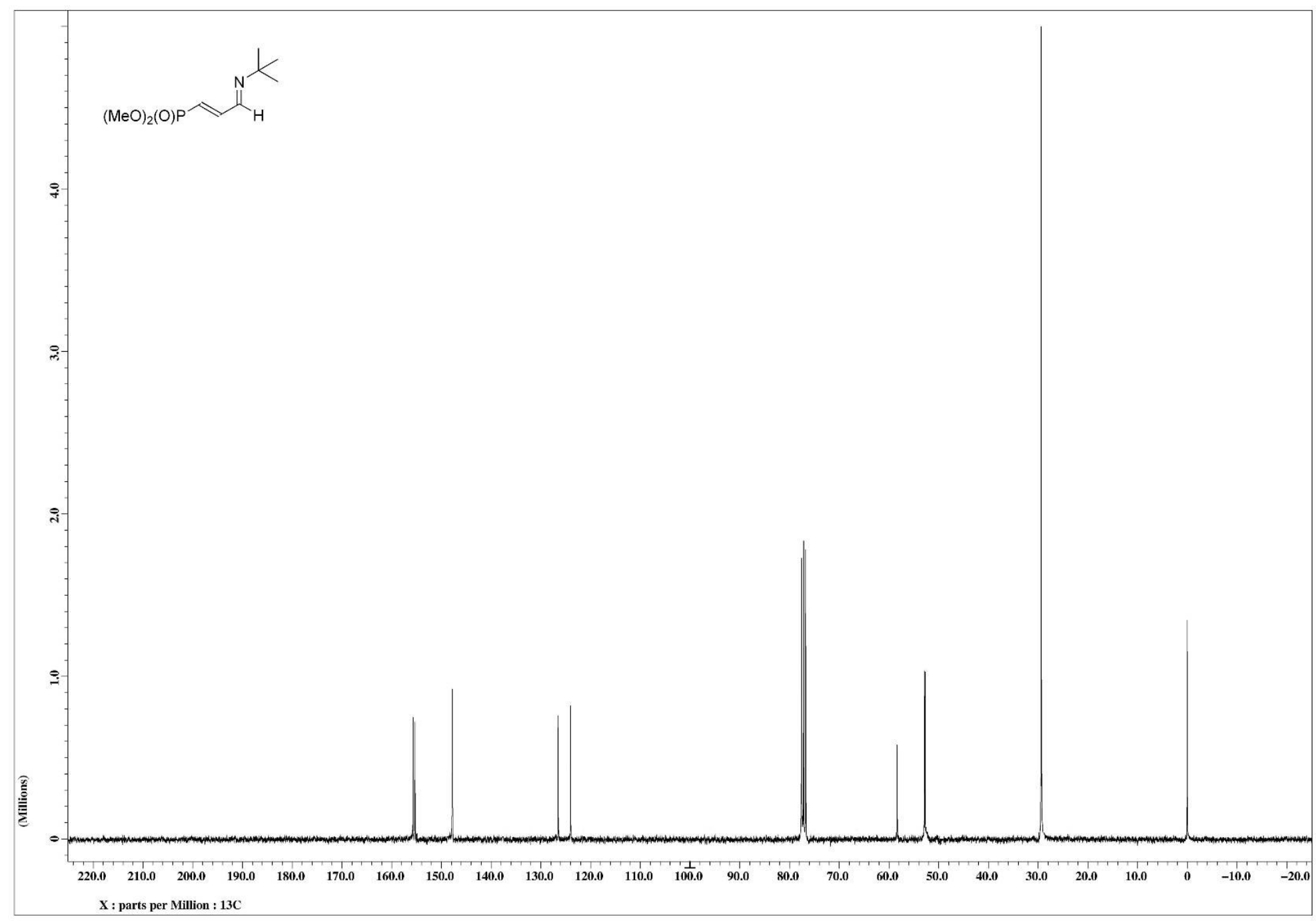


Dimethyl (1E,3E)-3-(isopropylimino)prop-1-enylphosphonate $1 \mathrm{~b}\left({ }^{1} \mathrm{H}-\mathrm{NMR}\right.$ : $\left.300 \mathrm{MHz}, \mathrm{CDCl}_{3}\right)$

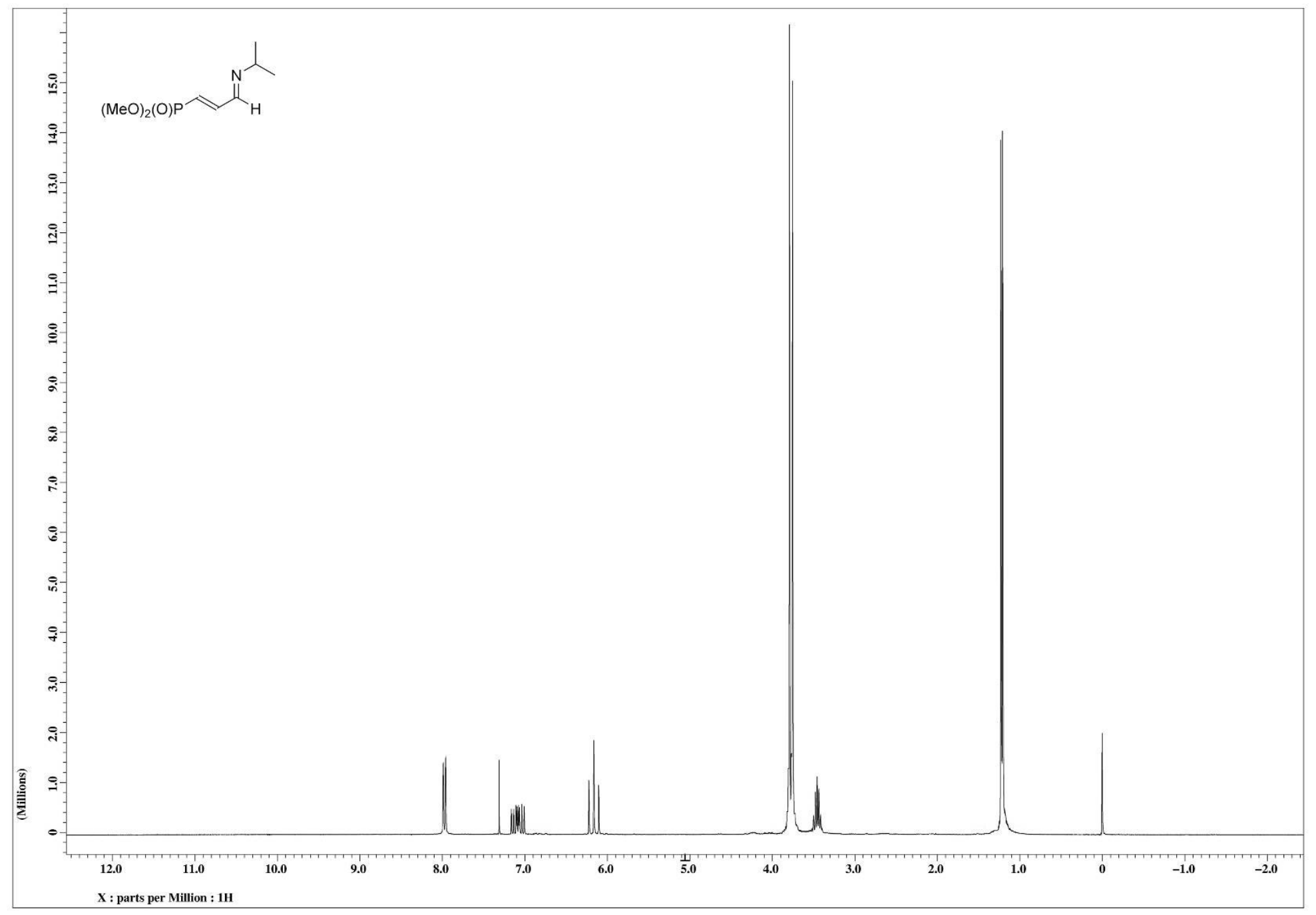

S 14 
Dimethyl (1E,3E)-3-(isopropylimino)prop-1-enylphosphonate $1 \mathrm{~b}\left({ }^{13} \mathrm{C}-\mathrm{NMR}\right.$ : $\left.75 \mathrm{MHz}, \mathrm{CDCl}_{3}\right)$

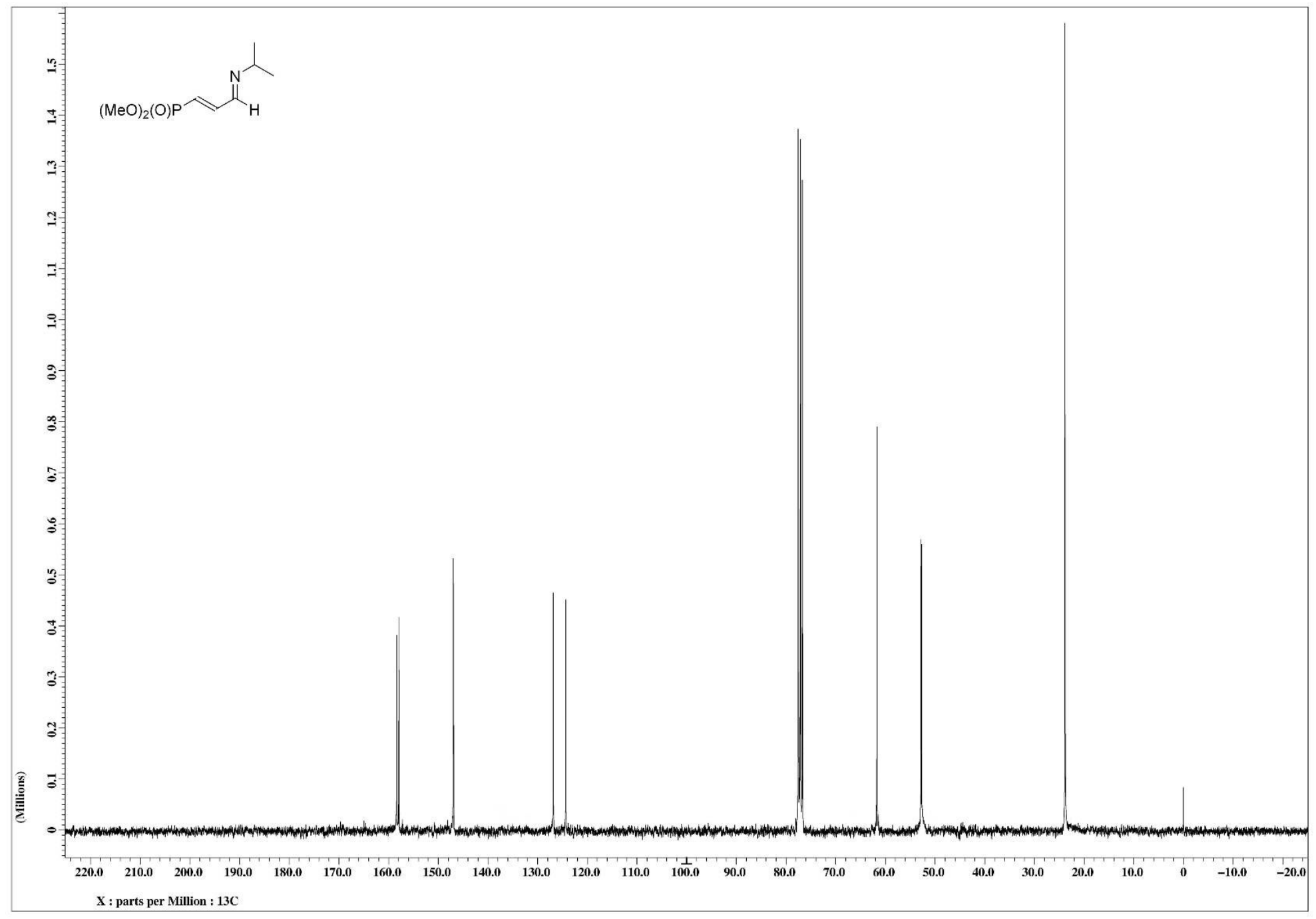

S 15 
Dimethyl (1E,3E)-3-(sec-butylimino)prop-1-enylphosphonate 1c $\left({ }^{1} \mathrm{H}-\mathrm{NMR}\right.$ : $\left.300 \mathrm{MHz}, \mathrm{CDCl}_{3}\right)$

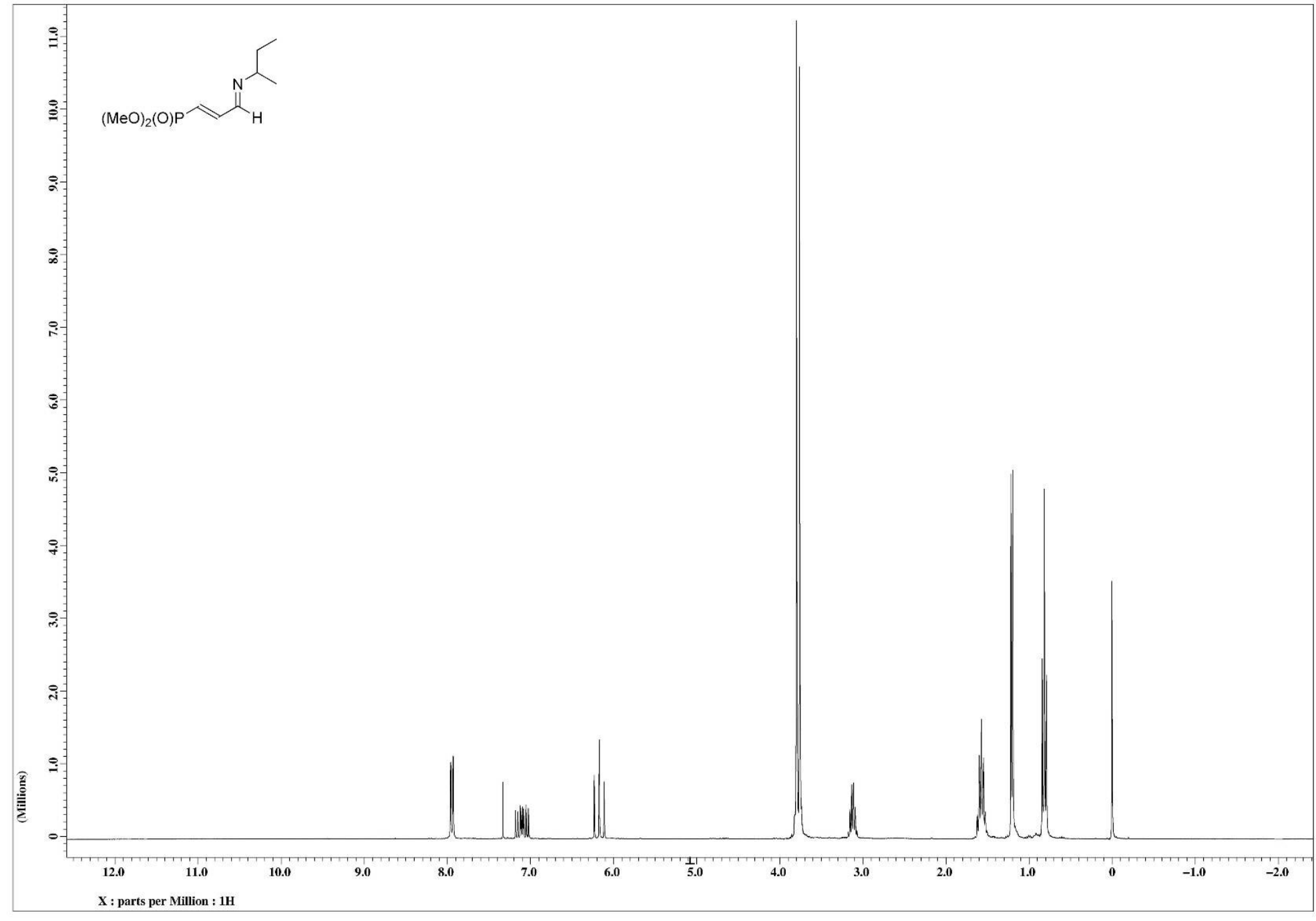


Dimethyl (1E,3E)-3-(sec-butylimino)prop-1-enylphosphonate $1 \mathrm{c}\left({ }^{13} \mathrm{C}-\mathrm{NMR}\right.$ : $\left.75 \mathrm{MHz}, \mathrm{CDCl}_{3}\right)$

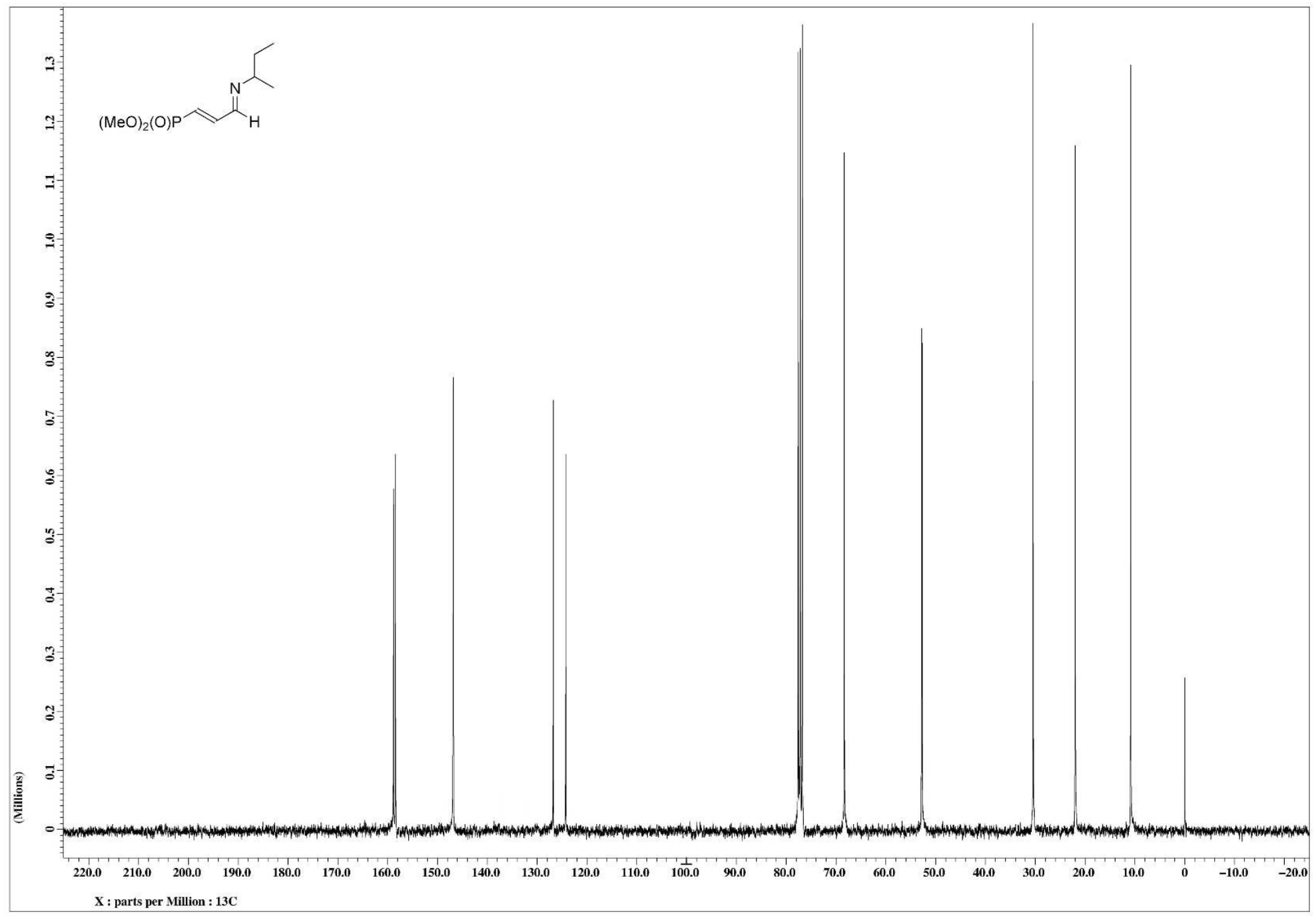

S 17 
Dimethyl (1E,3E)-3-(isobutylimino)prop-1-enylphosphonate 1d $\left({ }^{1} \mathrm{H}-\mathrm{NMR}\right.$ : $\left.300 \mathrm{MHz}, \mathrm{CDCl}_{3}\right)$

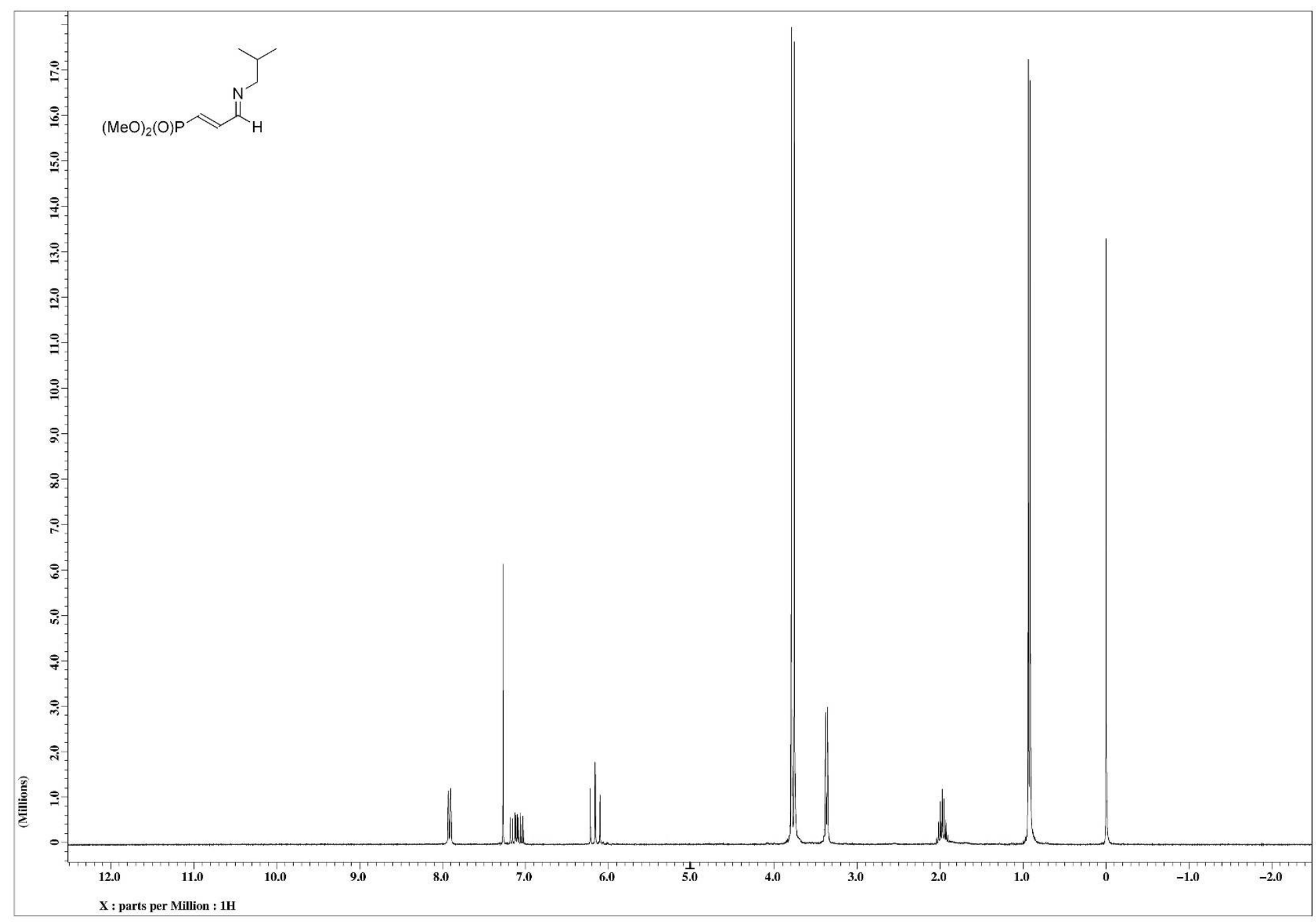

S 18 
Dimethyl (1E,3E)-3-(isobutylimino)prop-1-enylphosphonate $1 \mathrm{~d}\left({ }^{13} \mathrm{C}-\mathrm{NMR}\right.$ : $\left.75 \mathrm{MHz}, \mathrm{CDCl}_{3}\right)$

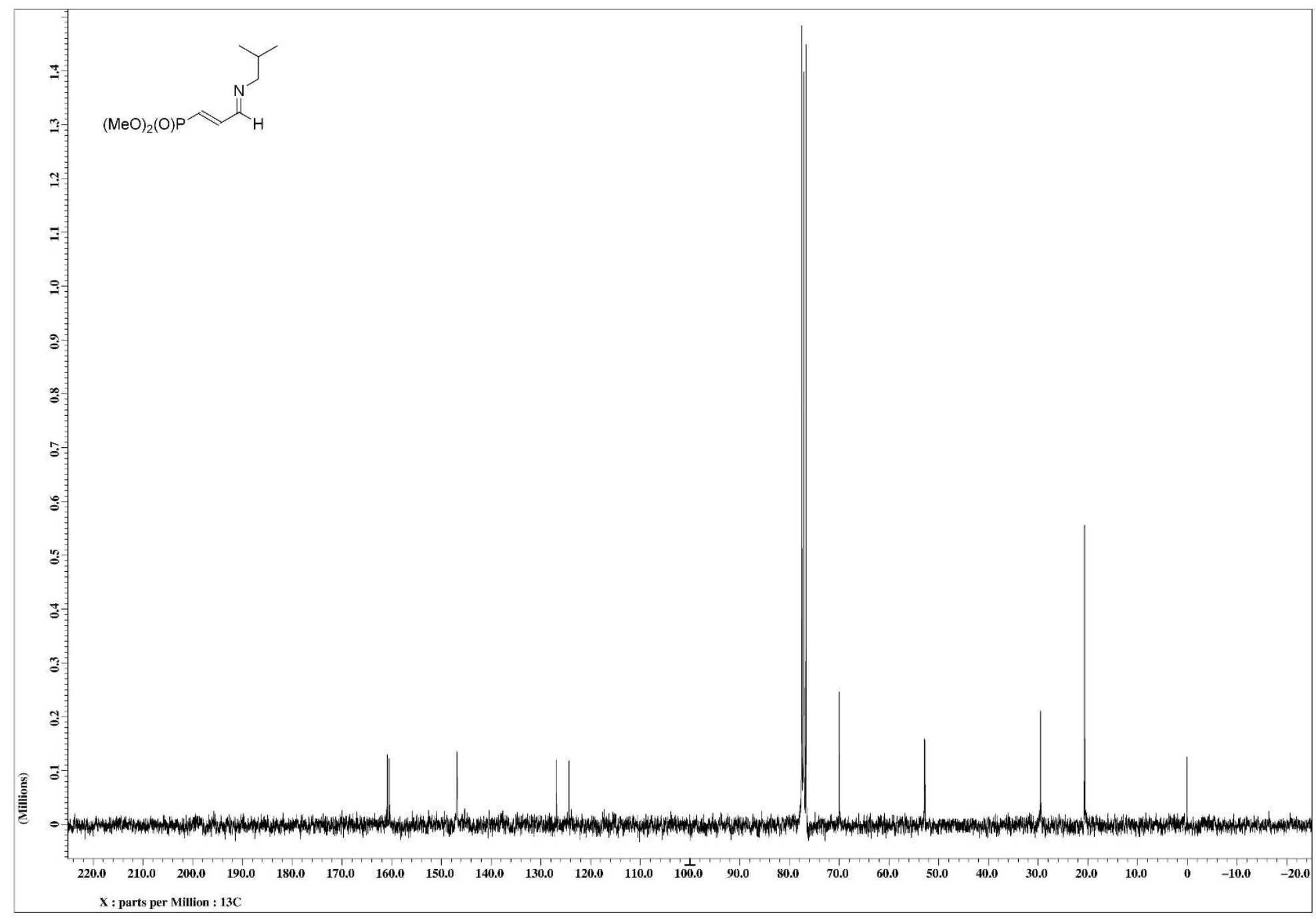


Dimethyl (1E,3E)-3-(cyclohexylimino)prop-1-enylphosphonate 1e $\left({ }^{1} \mathrm{H}-\mathrm{NMR}\right.$ : $\left.300 \mathrm{MHz}, \mathrm{CDCl}_{3}\right)$

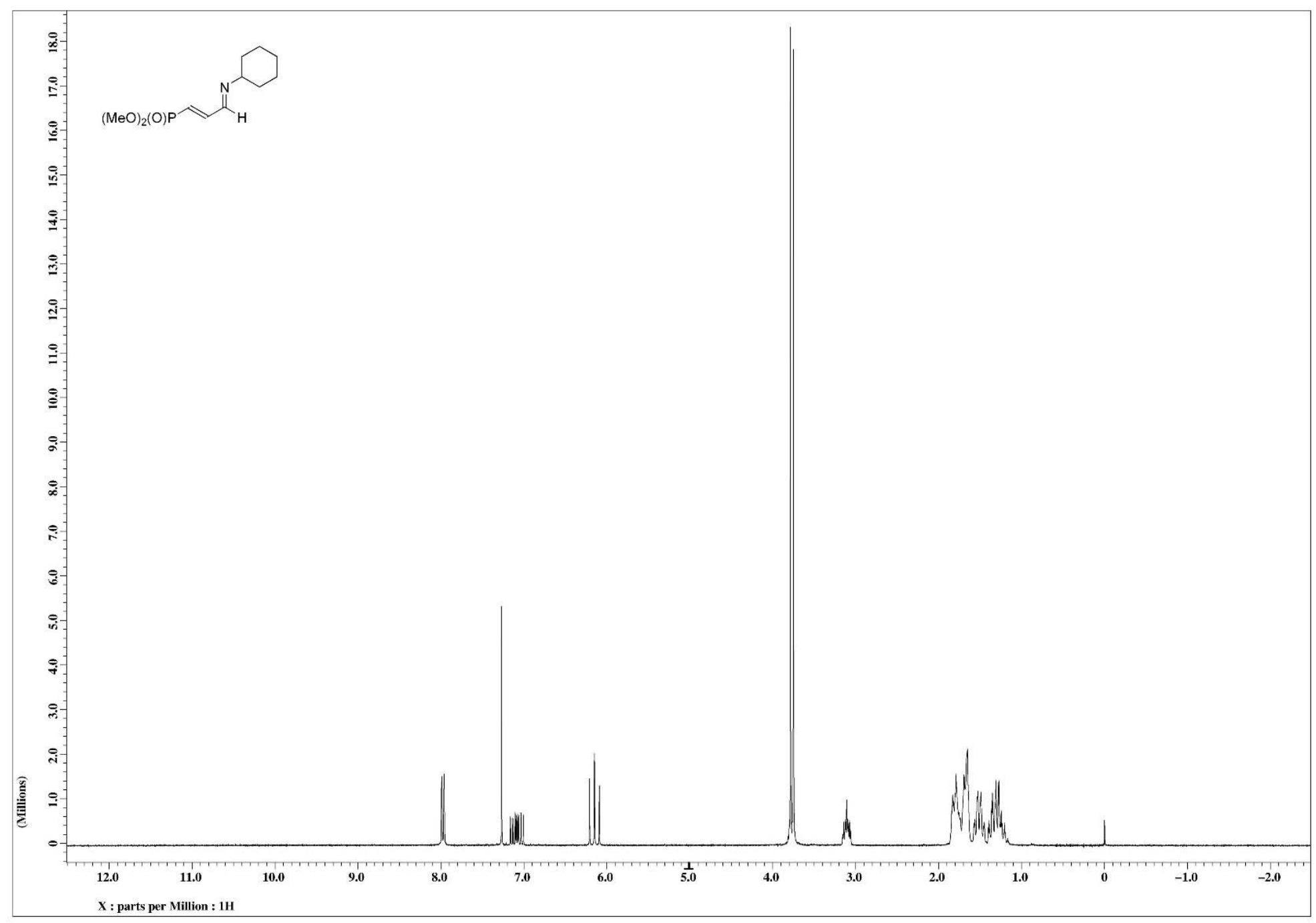


Dimethyl (1E,3E)-3-(cyclohexylimino)prop-1-enylphosphonate $1 \mathrm{e}\left({ }^{13} \mathrm{C}-\mathrm{NMR}\right.$ : $\left.75 \mathrm{MHz}, \mathrm{CDCl}_{3}\right)$

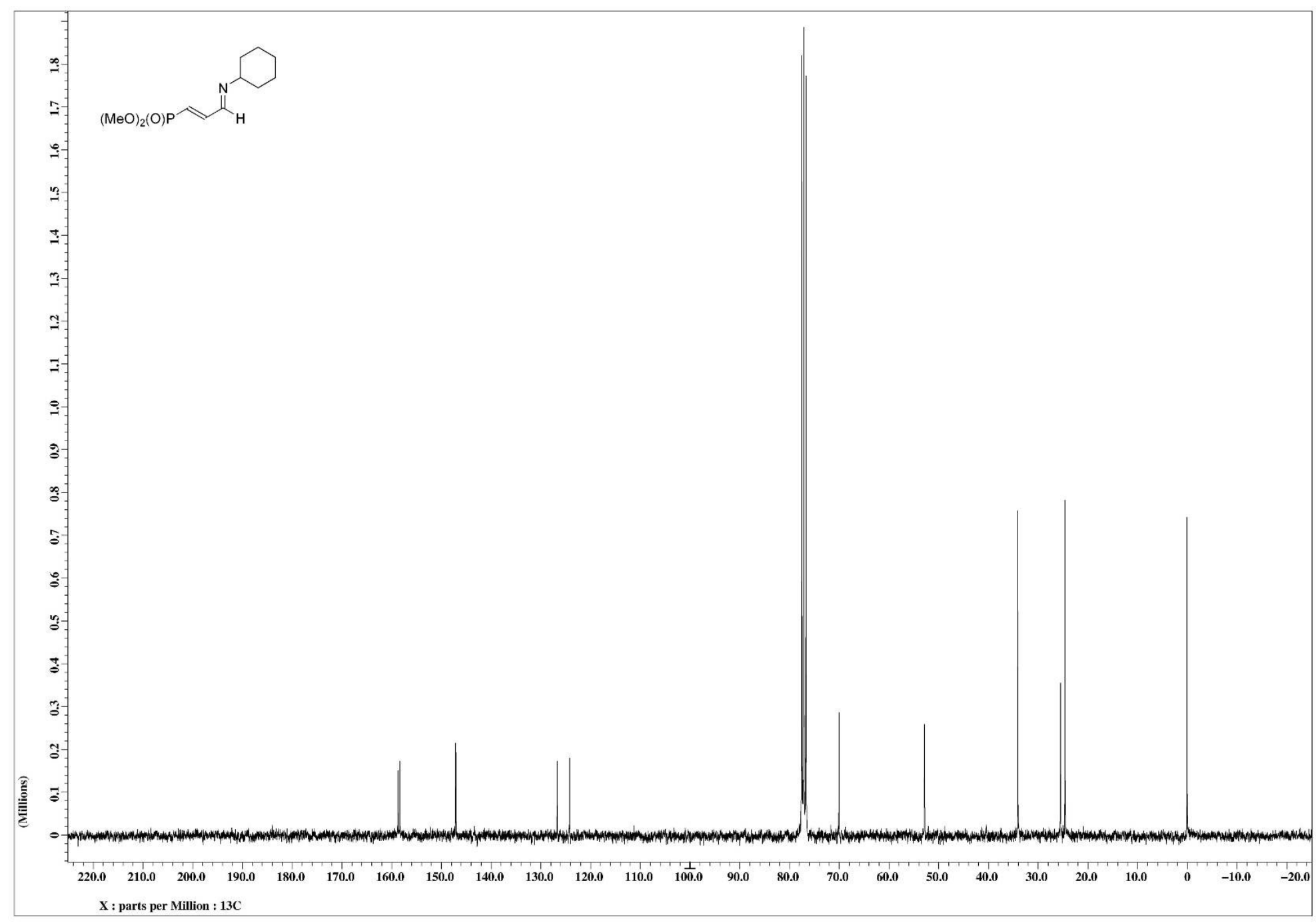


Dimethyl (1E,3E)-3-(benzylimino)prop-1-enylphosphonate 1f $\left({ }^{1} \mathrm{H}-\mathrm{NMR}\right.$ : $\left.300 \mathrm{MHz}, \mathrm{CDCl}_{3}\right)$

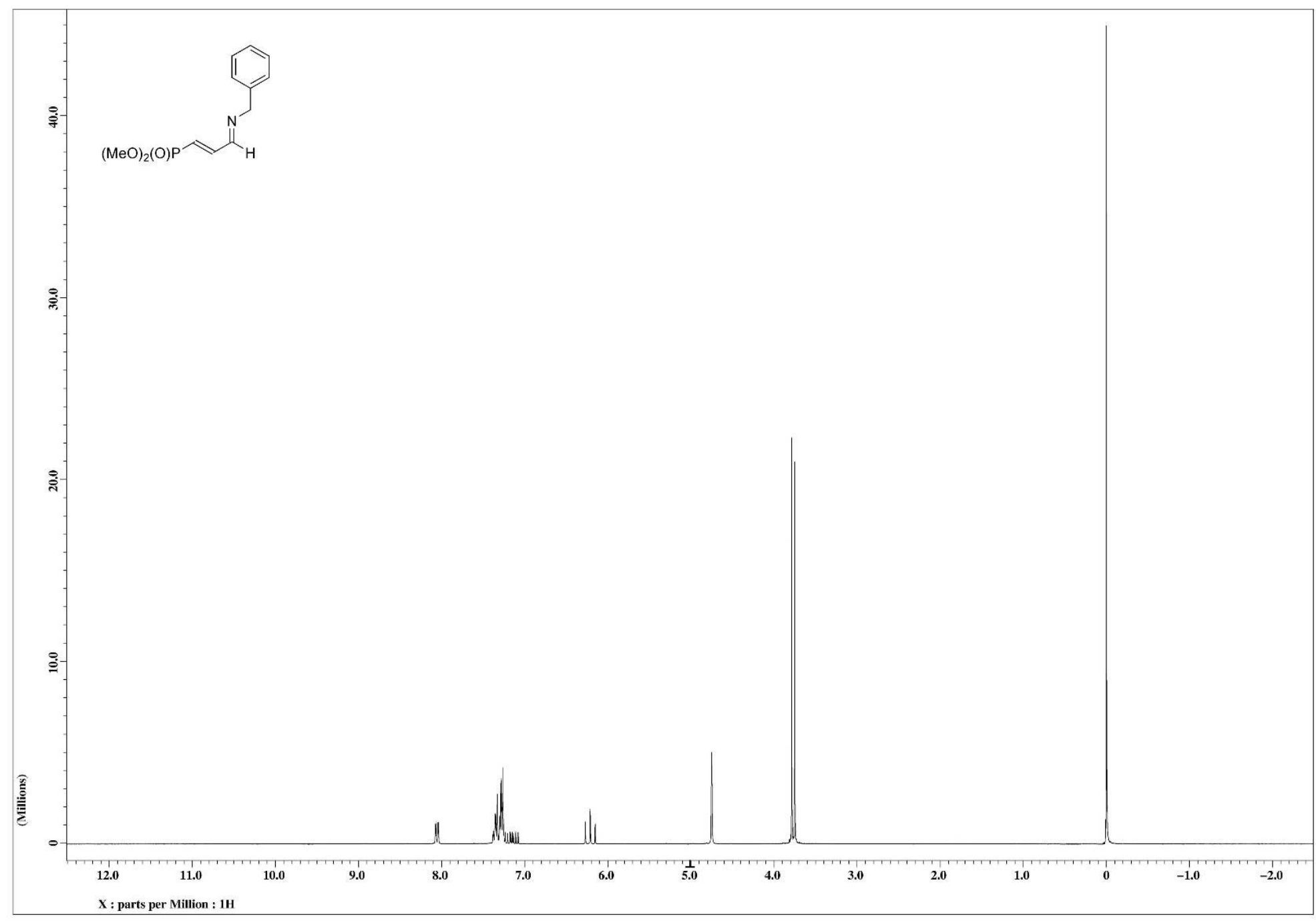


Dimethyl (1E,3E)-3-(benzylimino)prop-1-enylphosphonate $1 \mathrm{f}\left({ }^{13} \mathrm{C}-\mathrm{NMR}\right.$ : $\left.75 \mathrm{MHz}, \mathrm{CDCl}_{3}\right)$

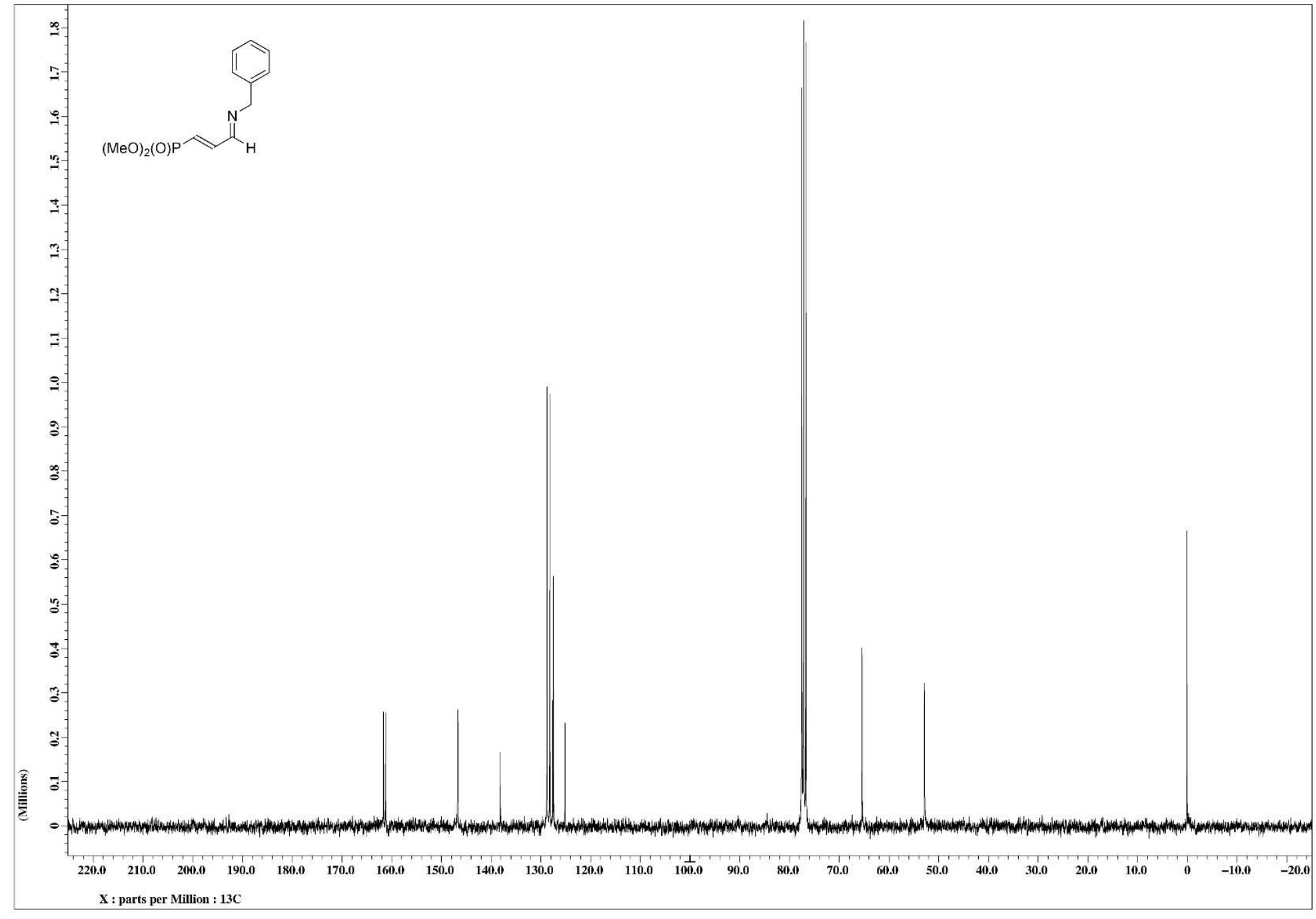


Diethyl (1E,3E)-3-(t-butylimino)prop-1-enylphosphonate $1 \mathrm{~g}\left({ }^{1} \mathrm{H}-\mathrm{NMR}\right.$ : $\left.300 \mathrm{MHz}, \mathrm{CDCl}_{3}\right)$

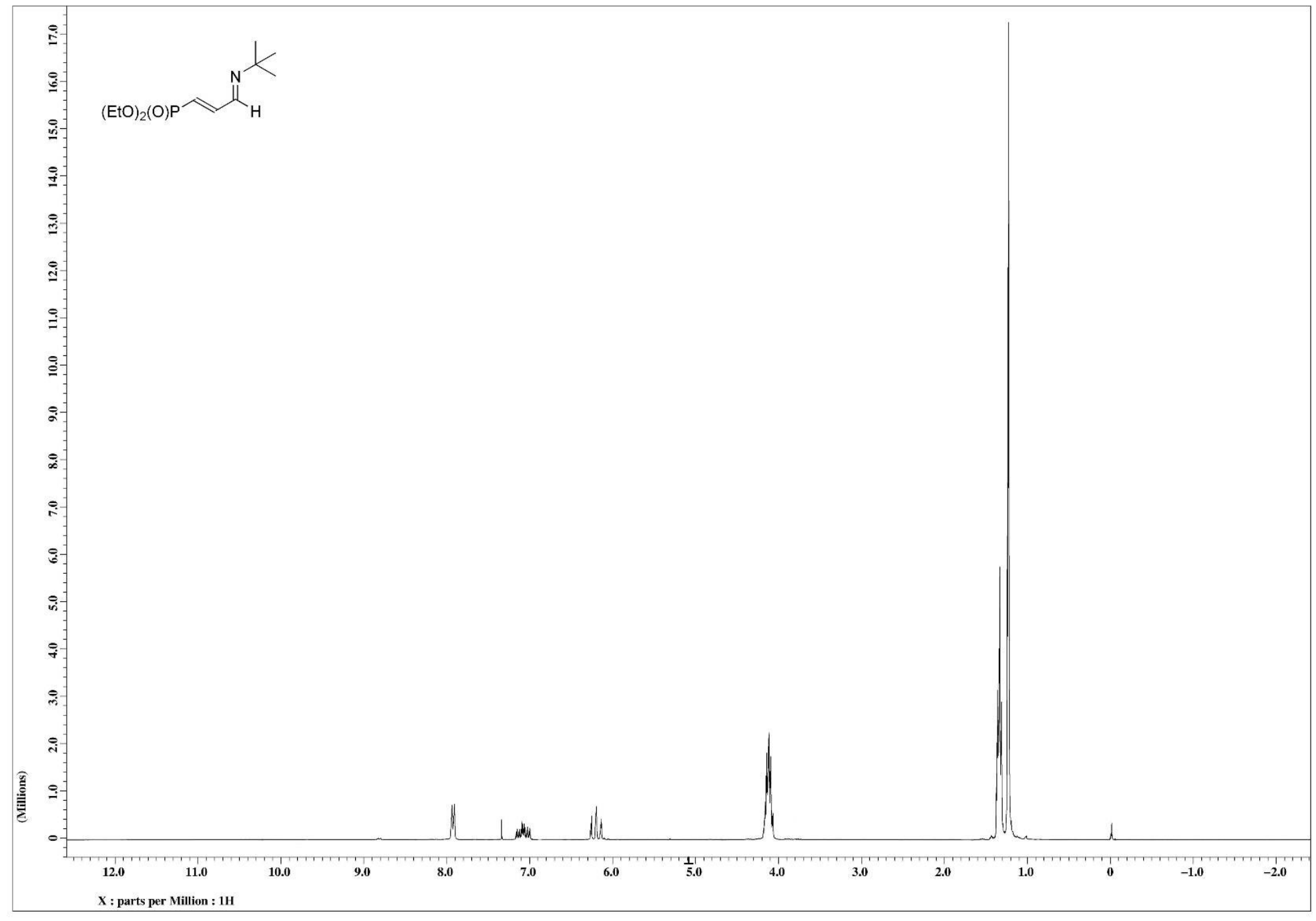


Diethyl (1E,3E)-3-(t-butylimino)prop-1-enylphosphonate $1 \mathrm{~g}\left({ }^{13} \mathrm{C}-\mathrm{NMR}\right.$ : $\left.75 \mathrm{MHz}, \mathrm{CDCl}_{3}\right)$

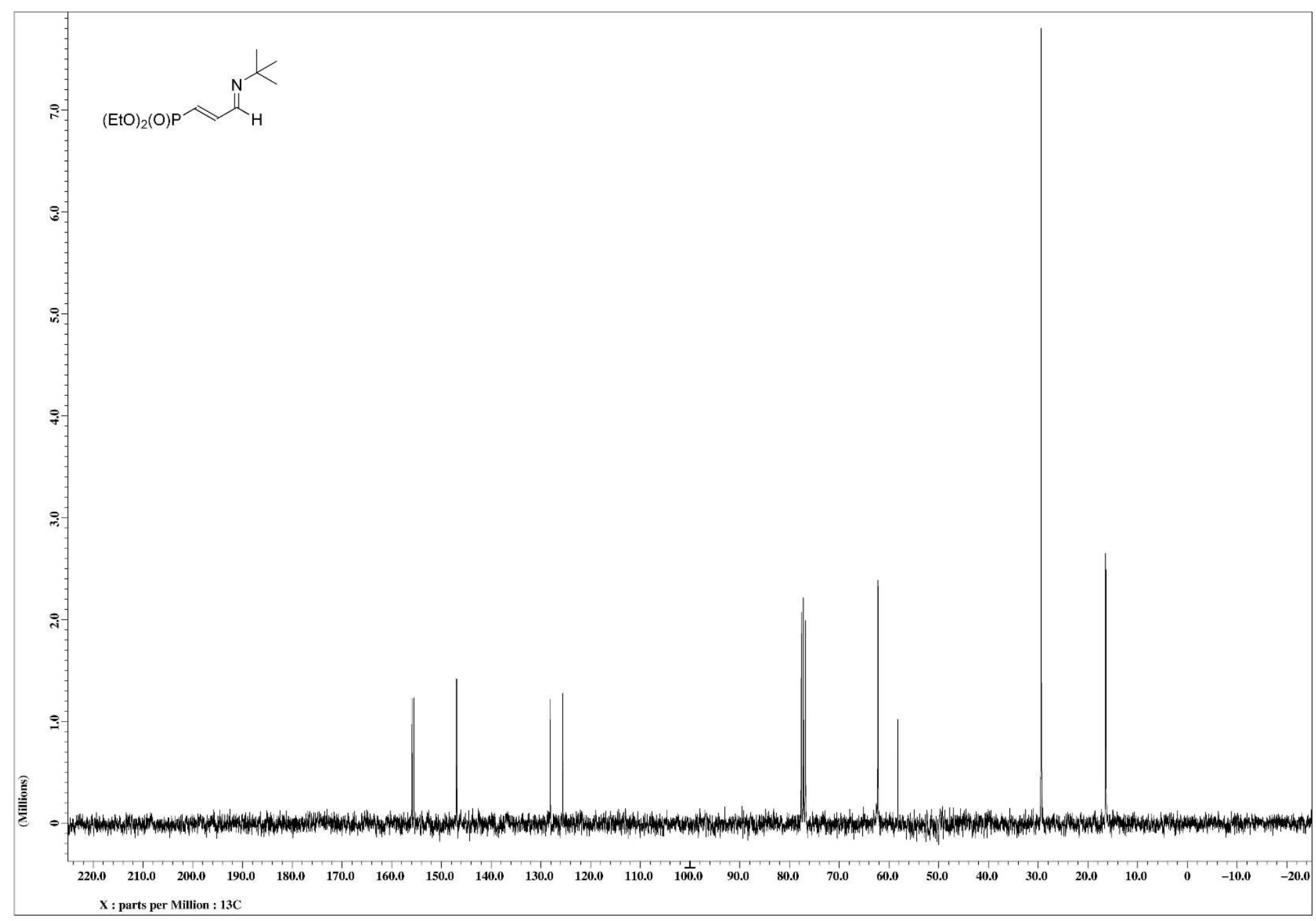


Diethyl (1E,3E)-3-(isopropylimino)prop-1-enylphosphonate $1 \mathrm{~h}\left({ }^{1} \mathrm{H}-\mathrm{NMR}\right.$ : $\left.300 \mathrm{MHz}, \mathrm{CDCl}_{3}\right)$

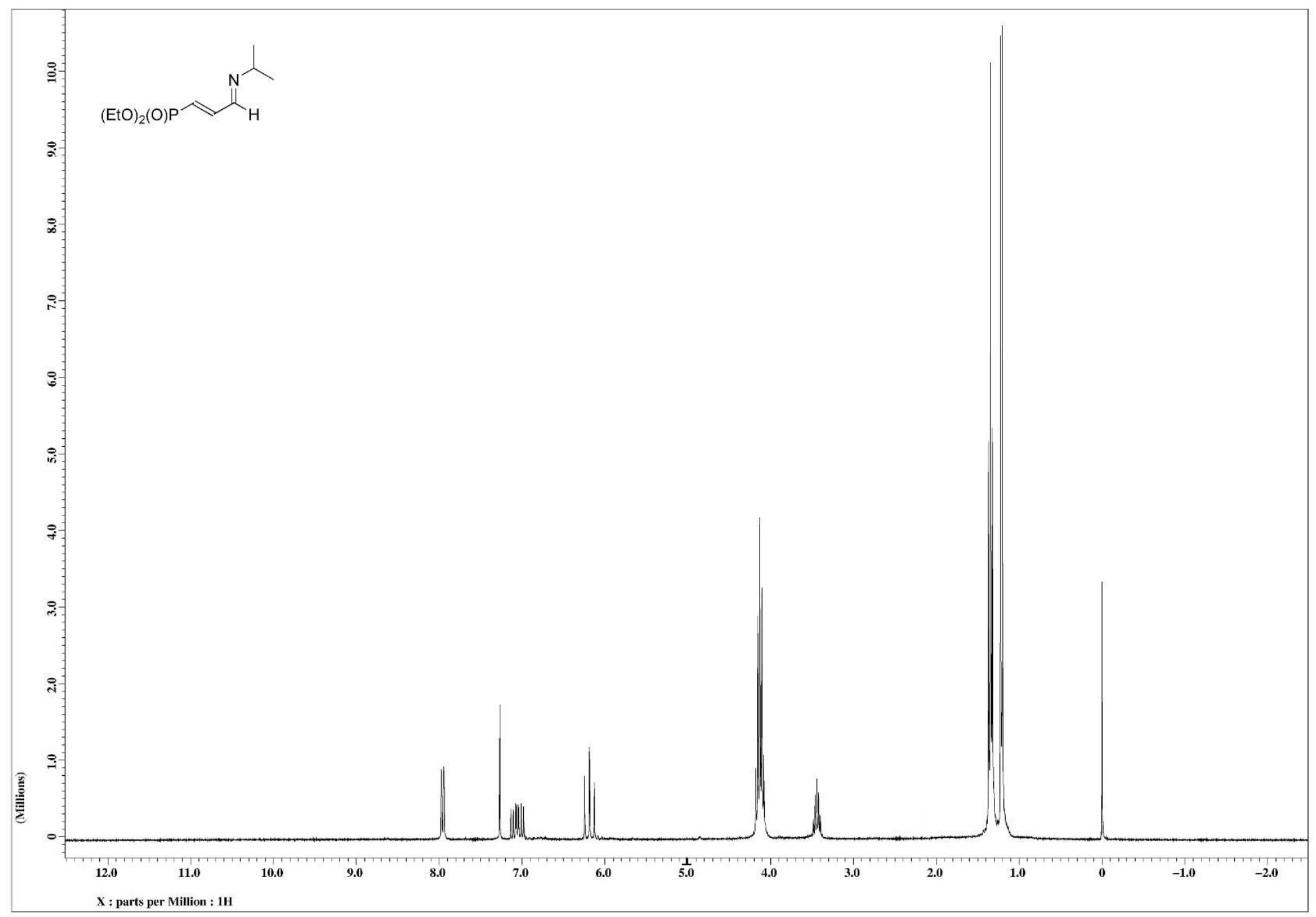

S 26 
Diethyl (1E,3E)-3-(isopropylimino)prop-1-enylphosphonate $1 \mathrm{~h}\left({ }^{13} \mathrm{C}-\mathrm{NMR}\right.$ : $\left.75 \mathrm{MHz}, \mathrm{CDCl}_{3}\right)$

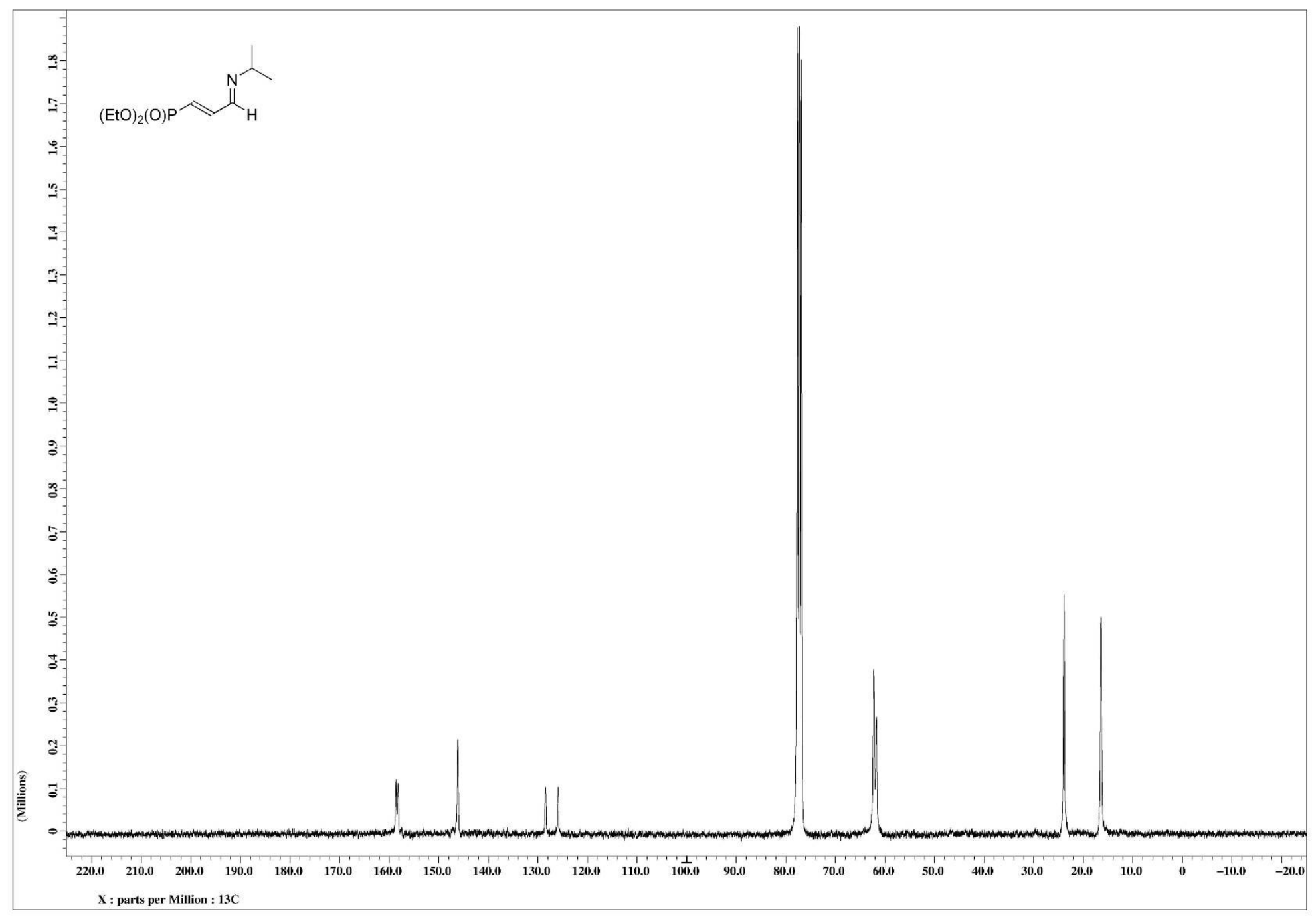

S27 
Diethyl (1E,3E)-3-(sec-butylimino)prop-1-enylphosphonate 1i ( ${ }^{1} \mathrm{H}-\mathrm{NMR}$ : $\left.300 \mathrm{MHz}, \mathrm{CDCl}_{3}\right)$

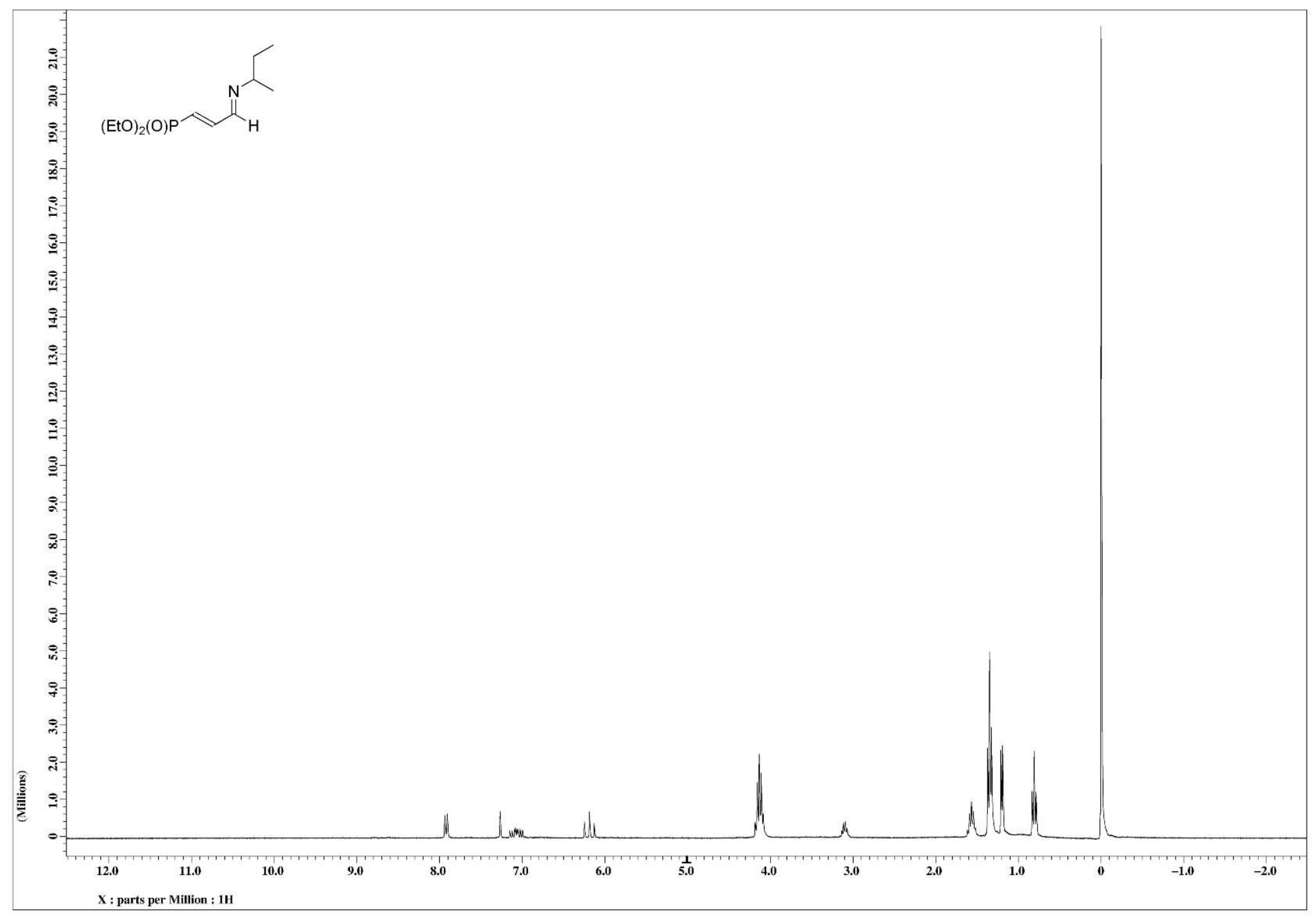

S 28 
Diethyl (1E,3E)-3-(sec-butylimino)prop-1-enylphosphonate 1i $\left({ }^{13} \mathrm{C}-\mathrm{NMR}\right.$ : $\left.75 \mathrm{MHz}, \mathrm{CDCl}_{3}\right)$

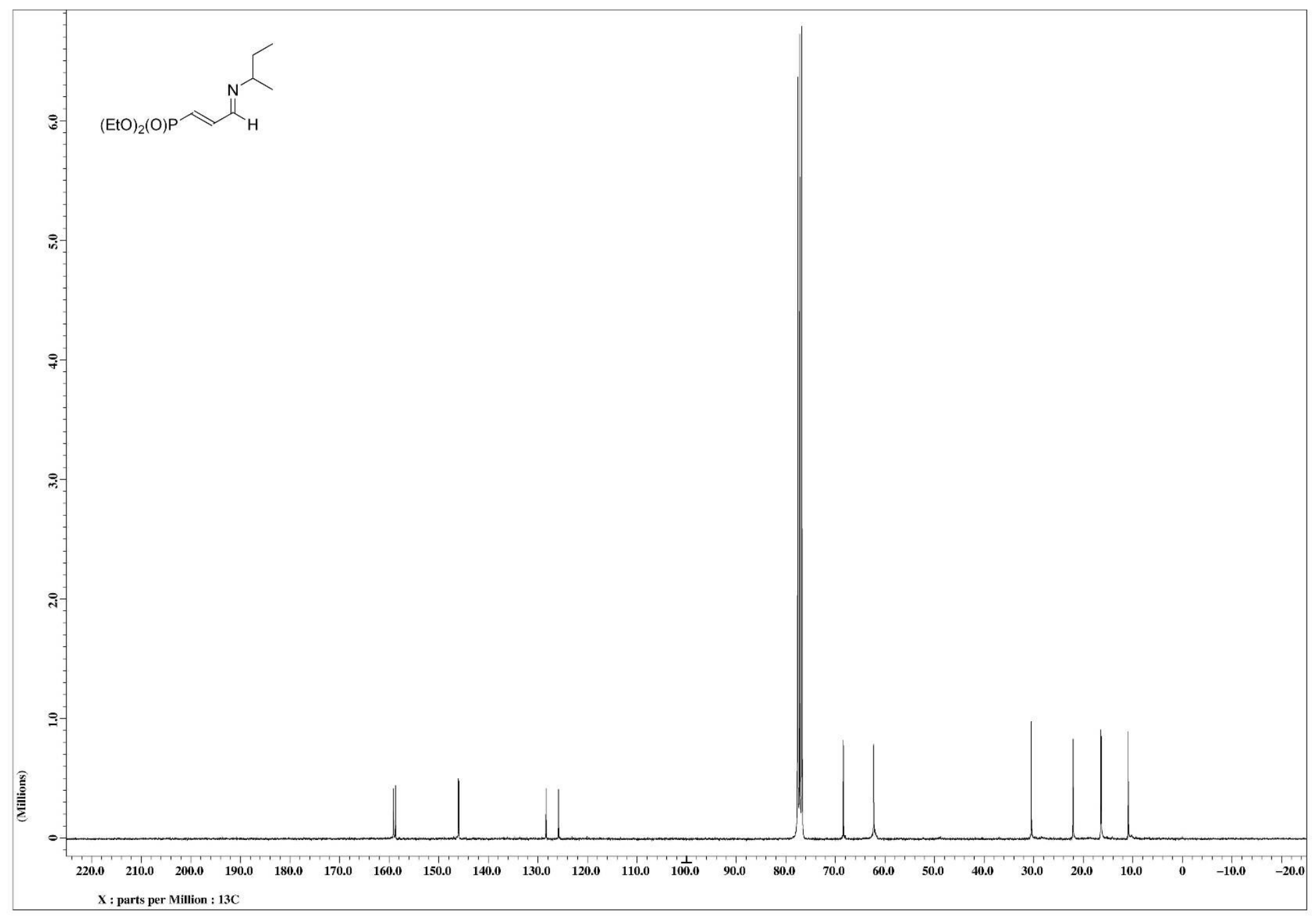

S 29 
Diethyl (1E,3E)-3-(isobutylimino)prop-1-enylphosphonate $1 \mathrm{j}\left({ }^{1} \mathrm{H}-\mathrm{NMR}\right.$ : $\left.300 \mathrm{MHz}, \mathrm{CDCl}_{3}\right)$

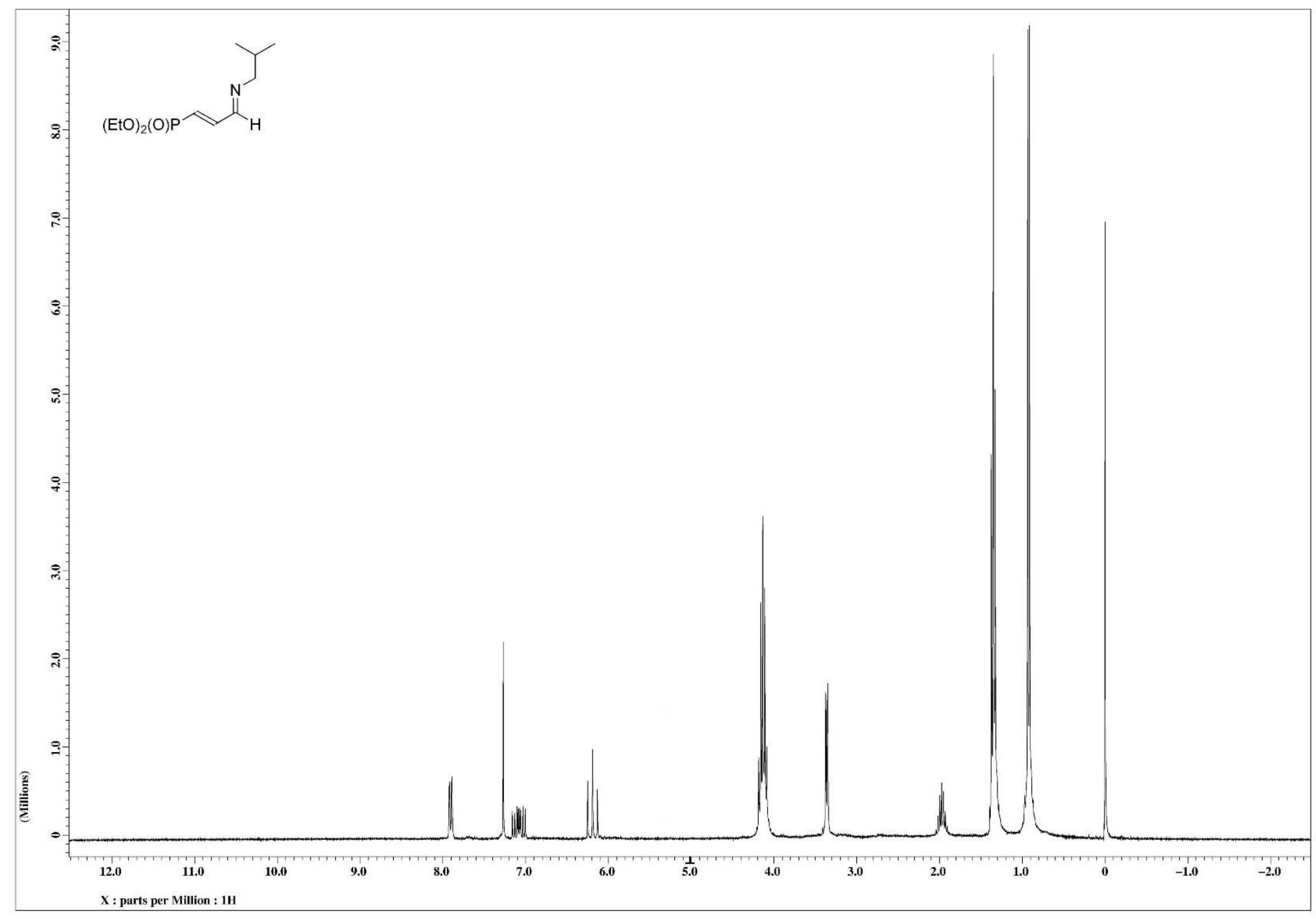

S 30 
Diethyl (1E,3E)-3-(isobutylimino)prop-1-enylphosphonate $1 \mathrm{j}\left({ }^{13} \mathrm{C}-\mathrm{NMR}\right.$ : $\left.75 \mathrm{MHz}, \mathrm{CDCl}_{3}\right)$

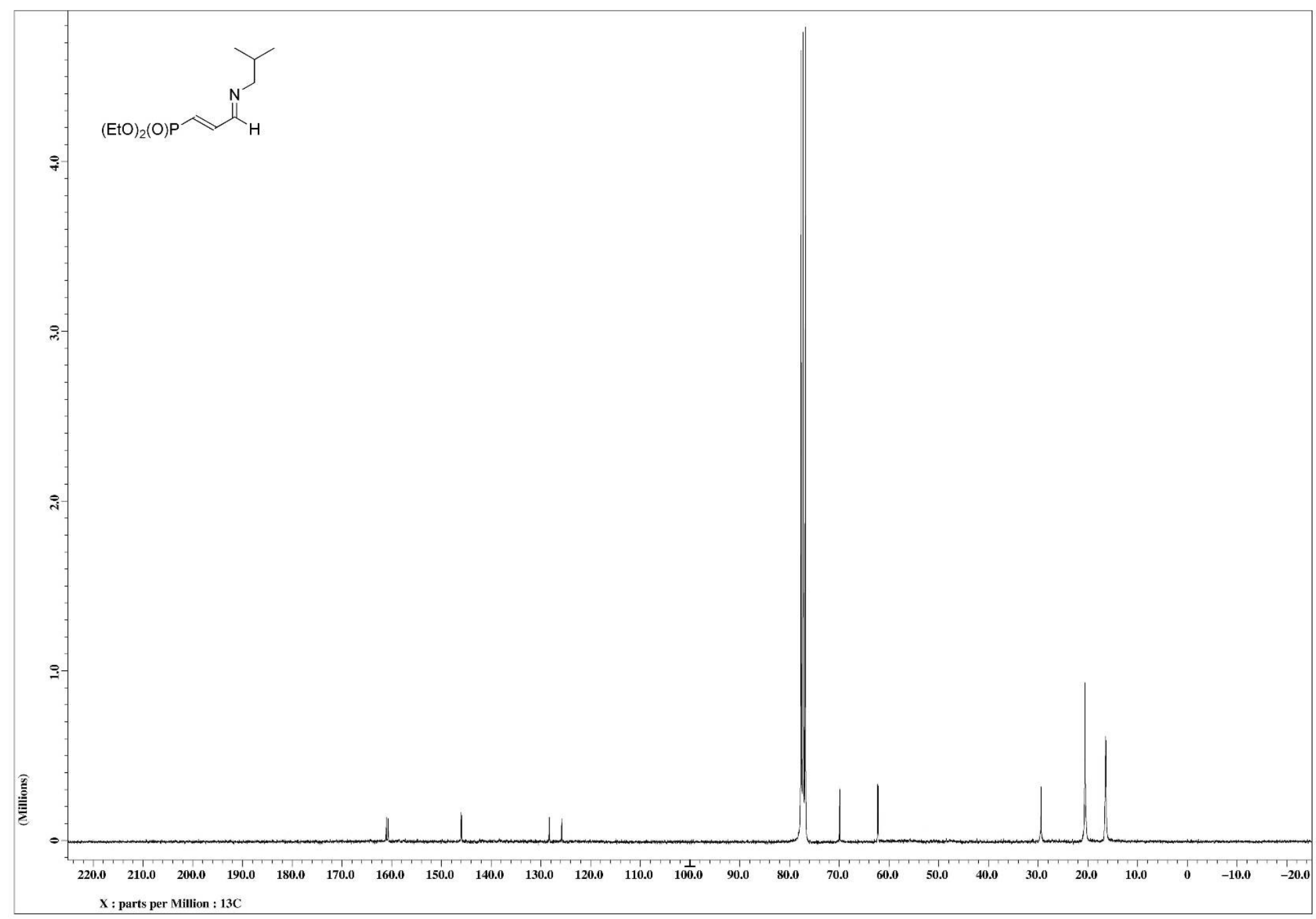

S 31 
Diethyl (1E,3E)-3-(cyclohexylimino)prop-1-enylphosphonate $1 \mathrm{k}\left({ }^{1} \mathrm{H}-\mathrm{NMR}\right.$ : $\left.300 \mathrm{MHz}, \mathrm{CDCl}_{3}\right)$

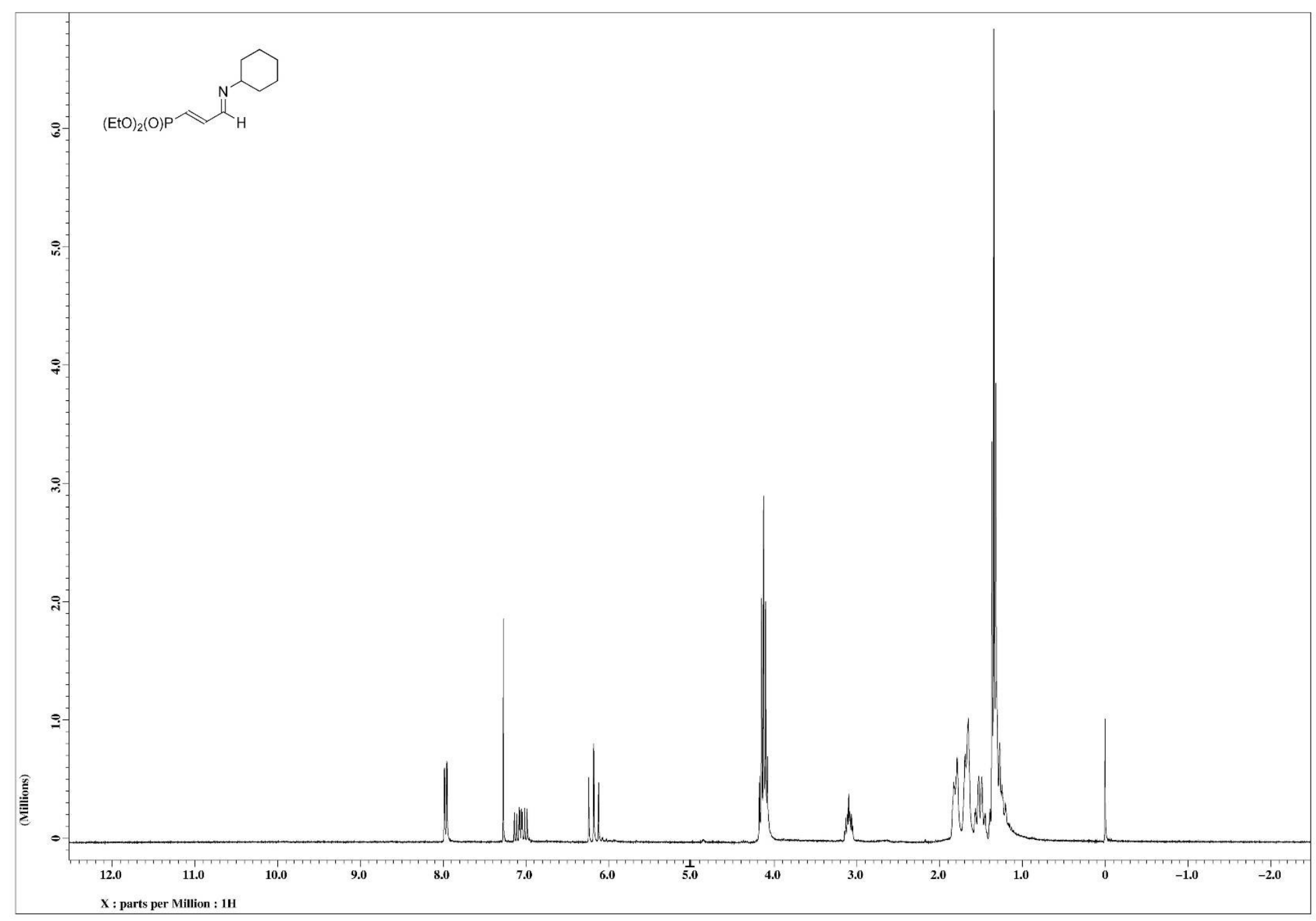


Diethyl (1E,3E)-3-(cyclohexylimino)prop-1-enylphosphonate $1 \mathrm{k}\left({ }^{13} \mathrm{C}-\mathrm{NMR}\right.$ : $\left.75 \mathrm{MHz}, \mathrm{CDCl}_{3}\right)$

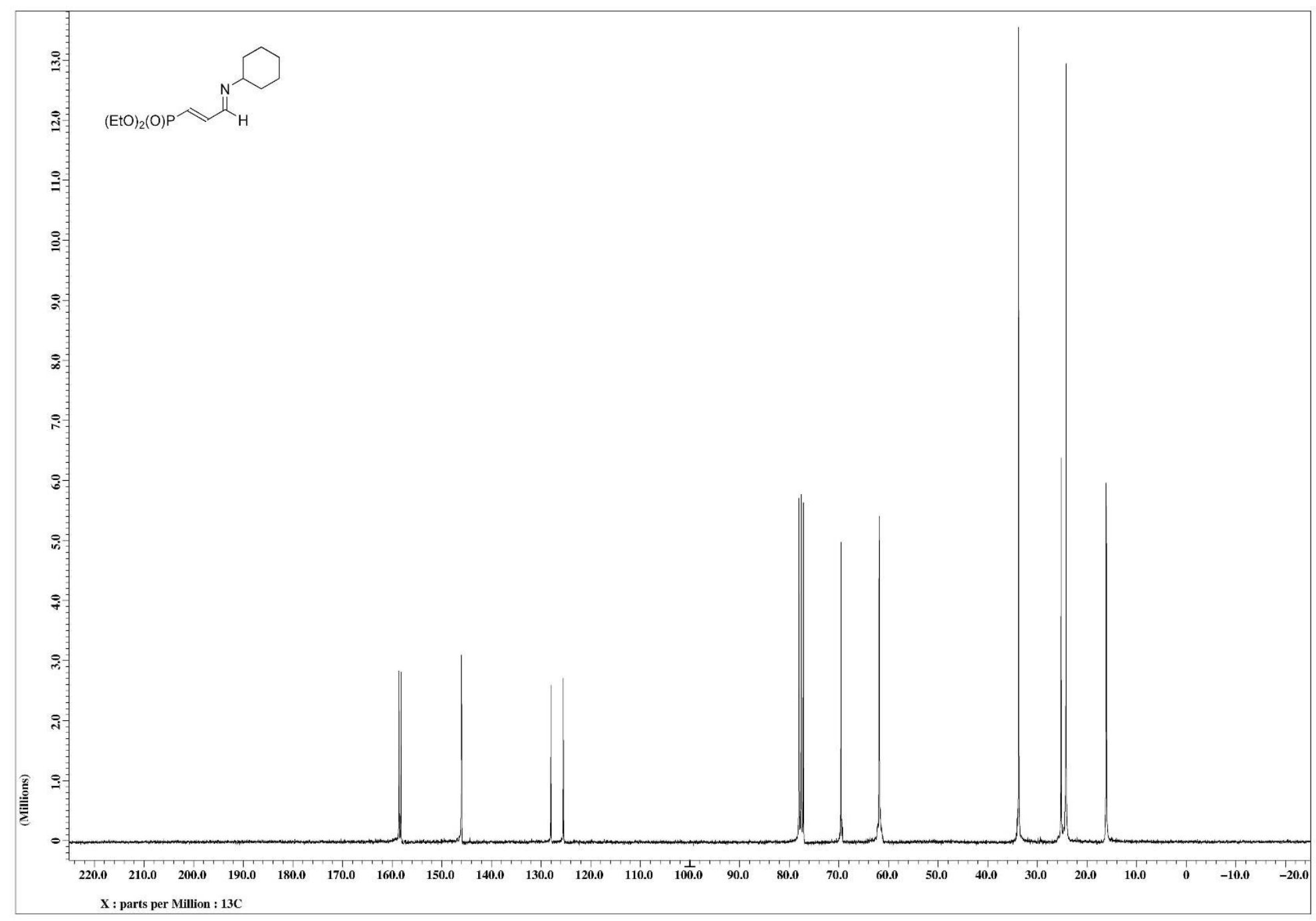


Dimethyl 2,3-epoxypropylphosphonate 3a $\left({ }^{1} \mathrm{H}-\mathrm{NMR}\right.$ : $\left.300 \mathrm{MHz}, \mathrm{CDCl}_{3}\right)$

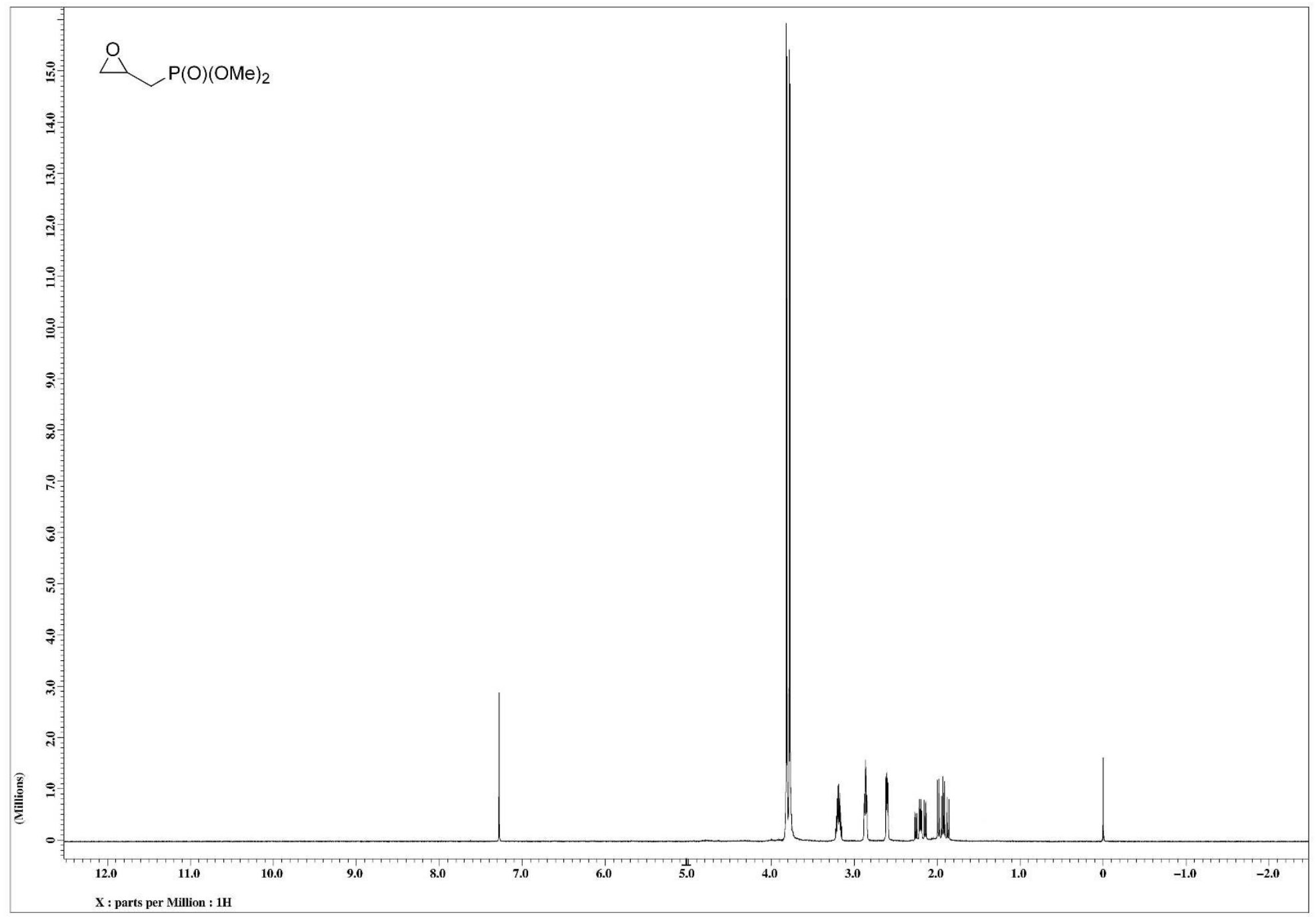

S 34 
Dimethyl 2,3-epoxypropylphosphonate 3a $\left({ }^{13} \mathrm{C}-\mathrm{NMR}\right.$ : $\left.75 \mathrm{MHz}, \mathrm{CDCl}_{3}\right)$

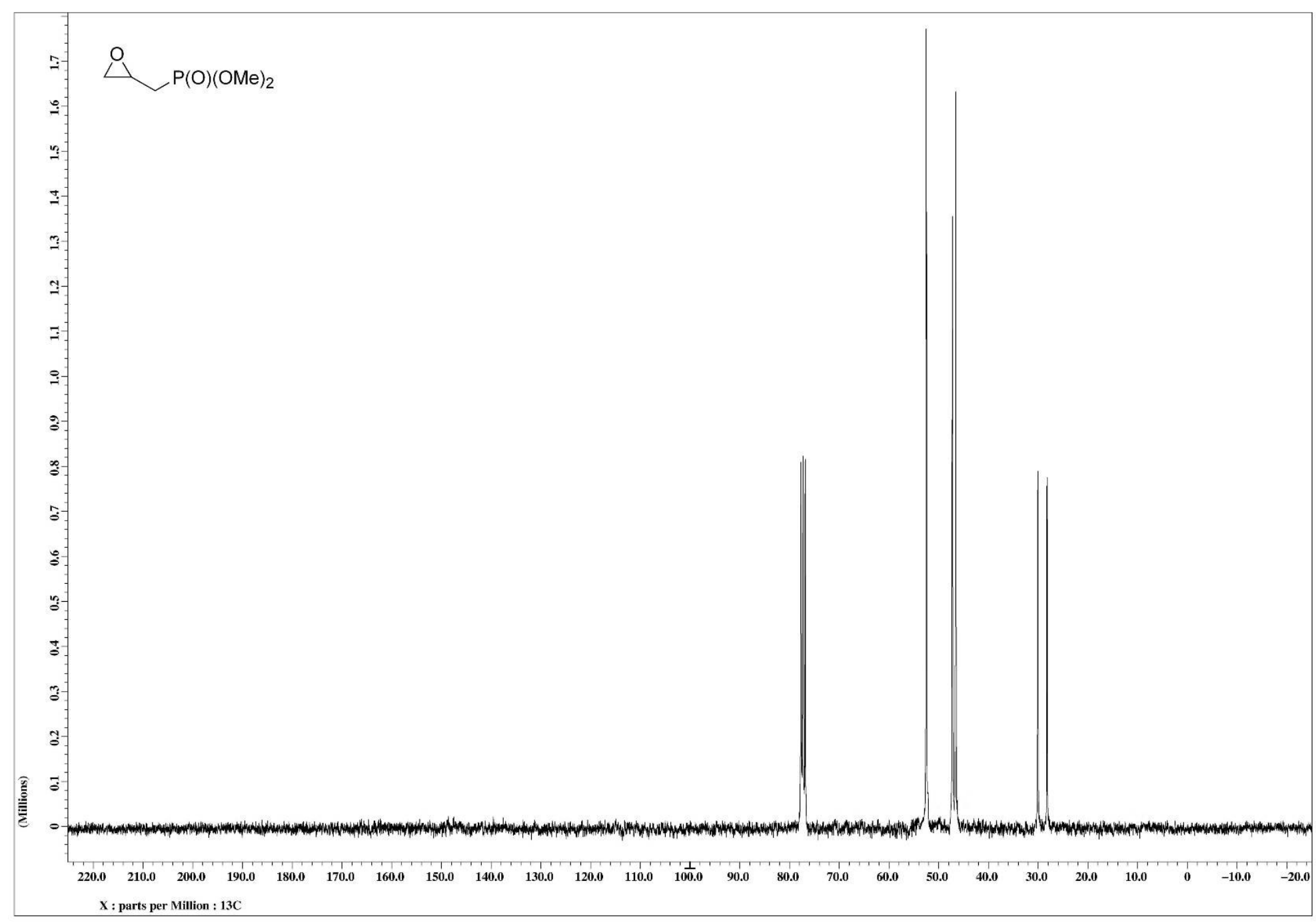


Dimethyl (1E)-3-oxoprop-1-enylphosphonate 4a ( ${ }^{1} \mathrm{H}-\mathrm{NMR}$ : $300 \mathrm{MHz}, \mathrm{CDCl}_{3}$ )

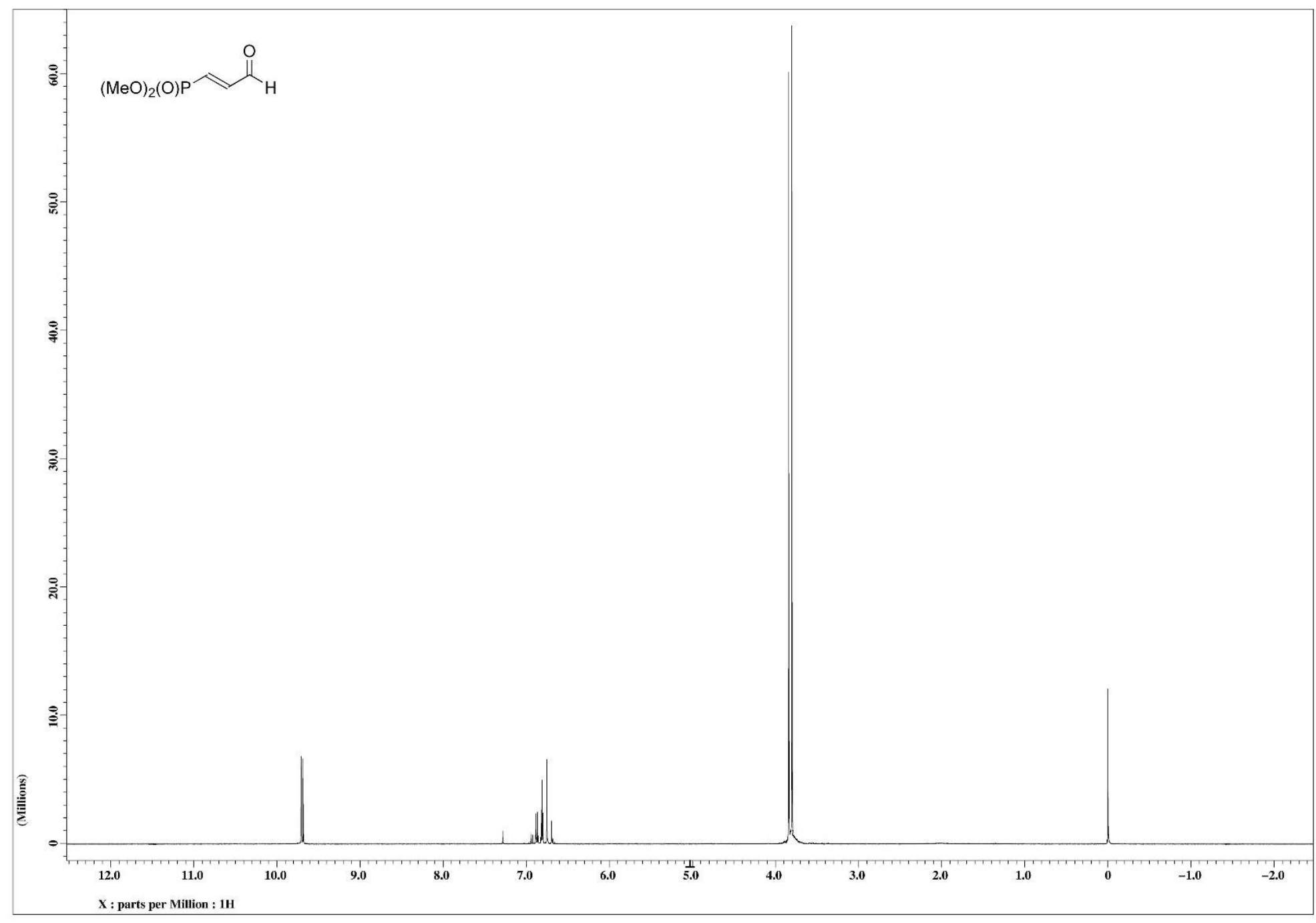

S 36 
Dimethyl (1E)-3-oxoprop-1-enylphosphonate 4a $\left({ }^{13} \mathrm{C}-\mathrm{NMR}\right.$ : $\left.75 \mathrm{MHz}, \mathrm{CDCl}_{3}\right)$

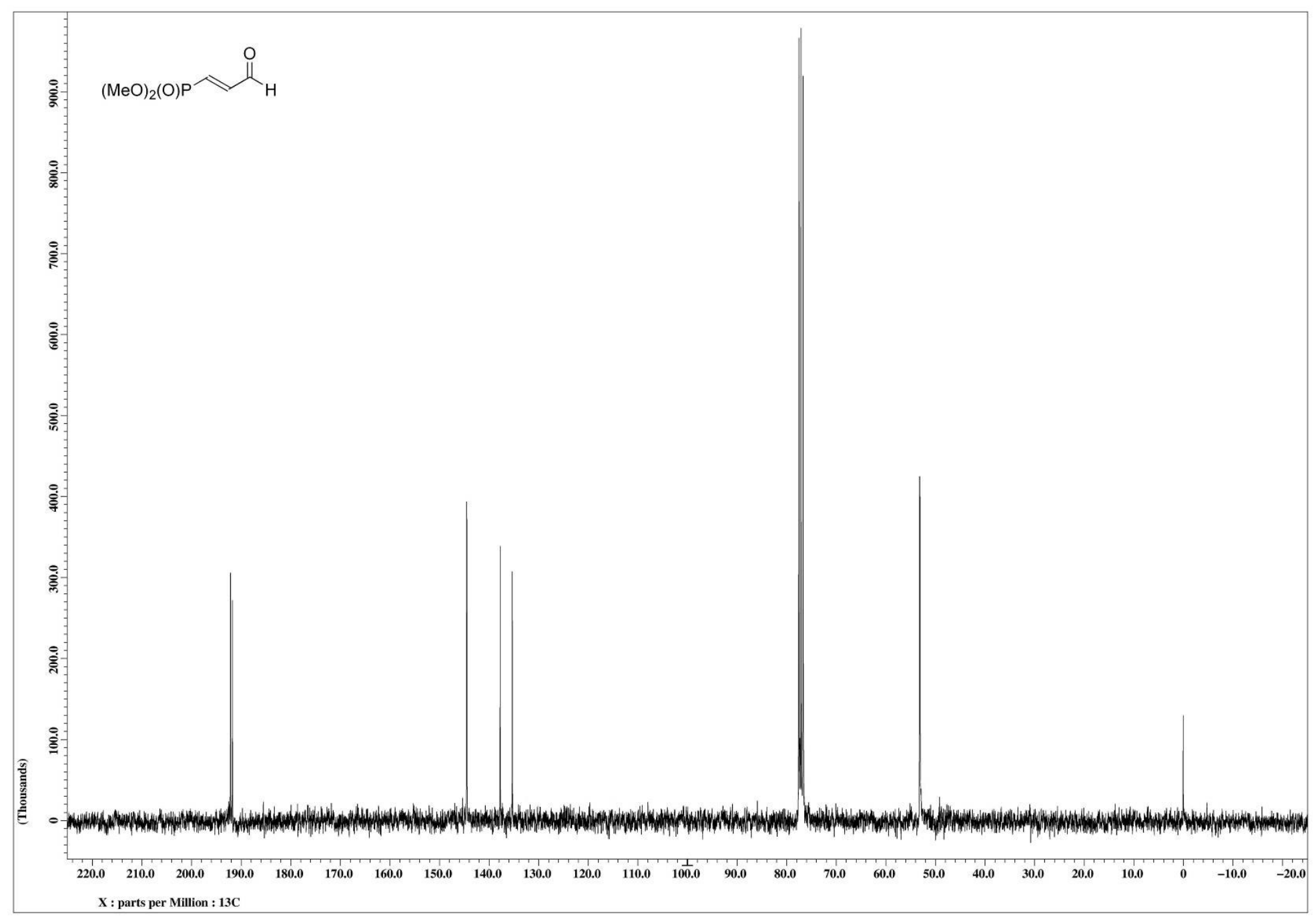

S37 
Diethyl (1E,3E)-3-\{[(4-methylphenyl)sulfinyl]imino\}prop-1-enylphosphonate $5\left({ }^{1} \mathrm{H}-\mathrm{NMR}\right.$ : $\left.300 \mathrm{MHz}, \mathrm{CDCl}_{3}\right)$

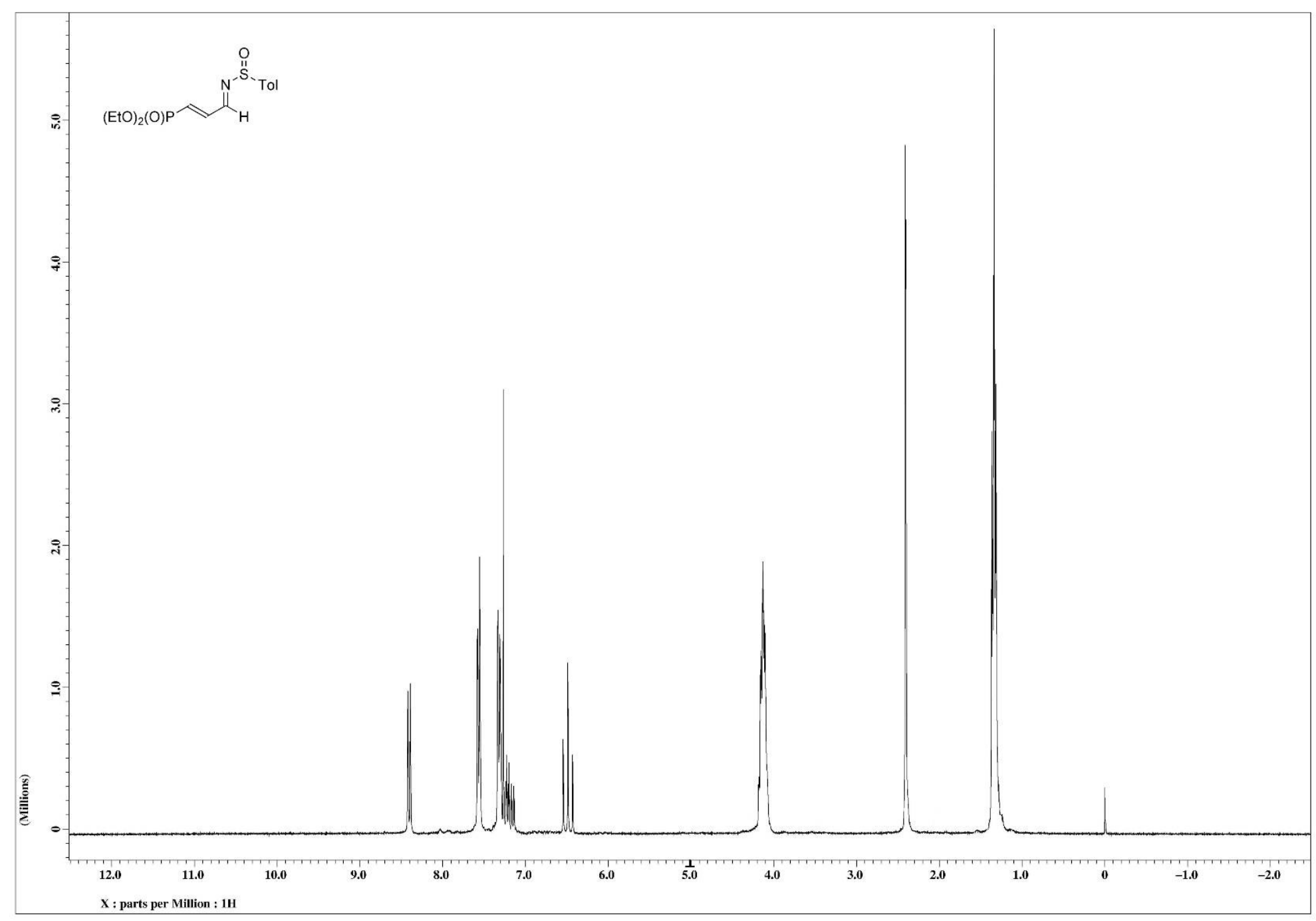

S 38 


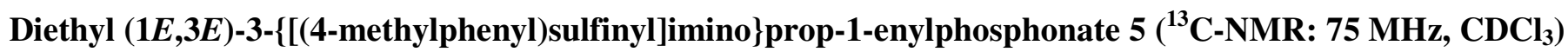

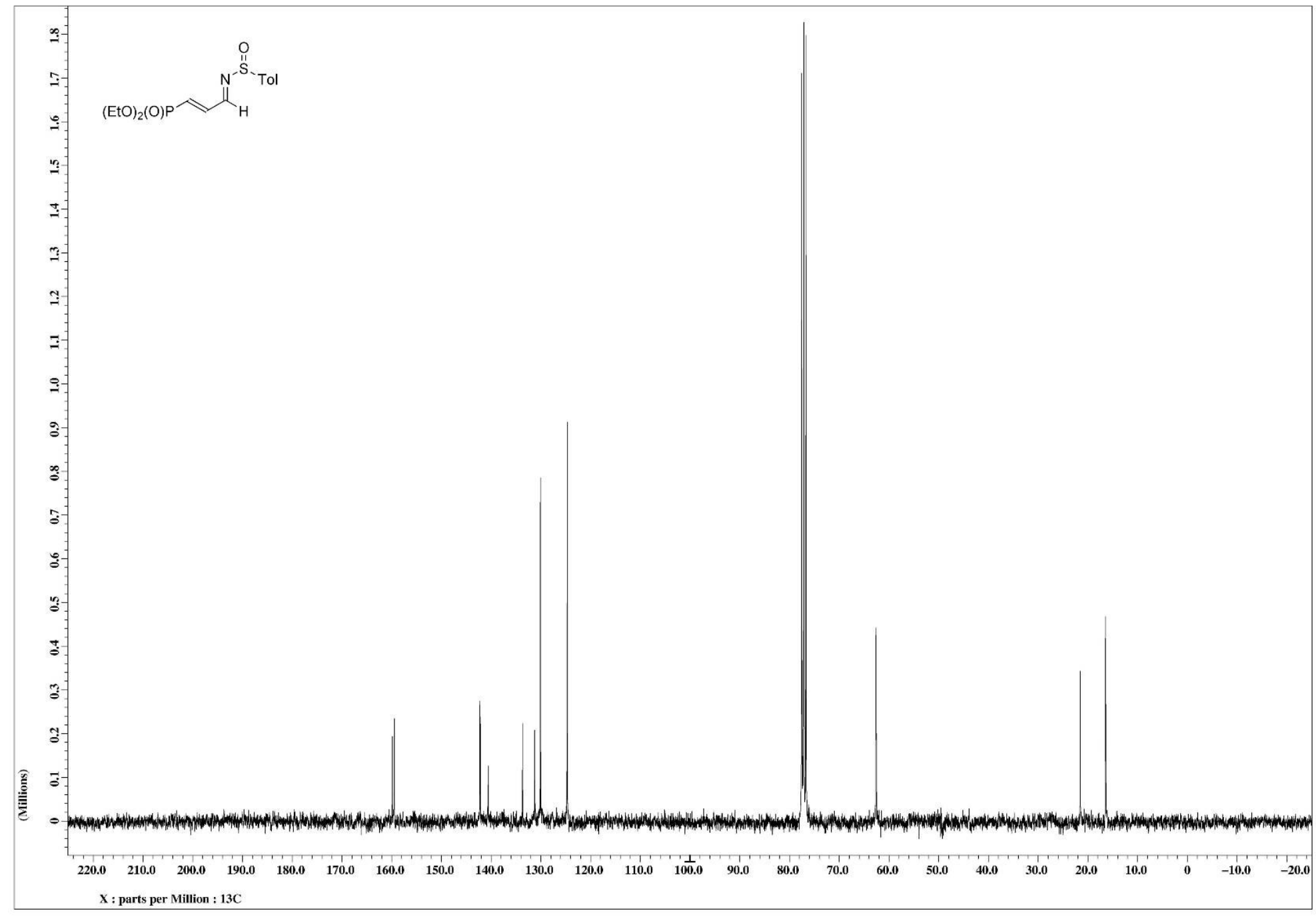


Dimethyl (1E,3E)-3-(dimethylhydrazono)prop-1-enylphosphonate 6a $\left({ }^{1} \mathrm{H}-\mathrm{NMR}: 300 \mathrm{MHz}, \mathrm{CDCl}_{3}\right)$

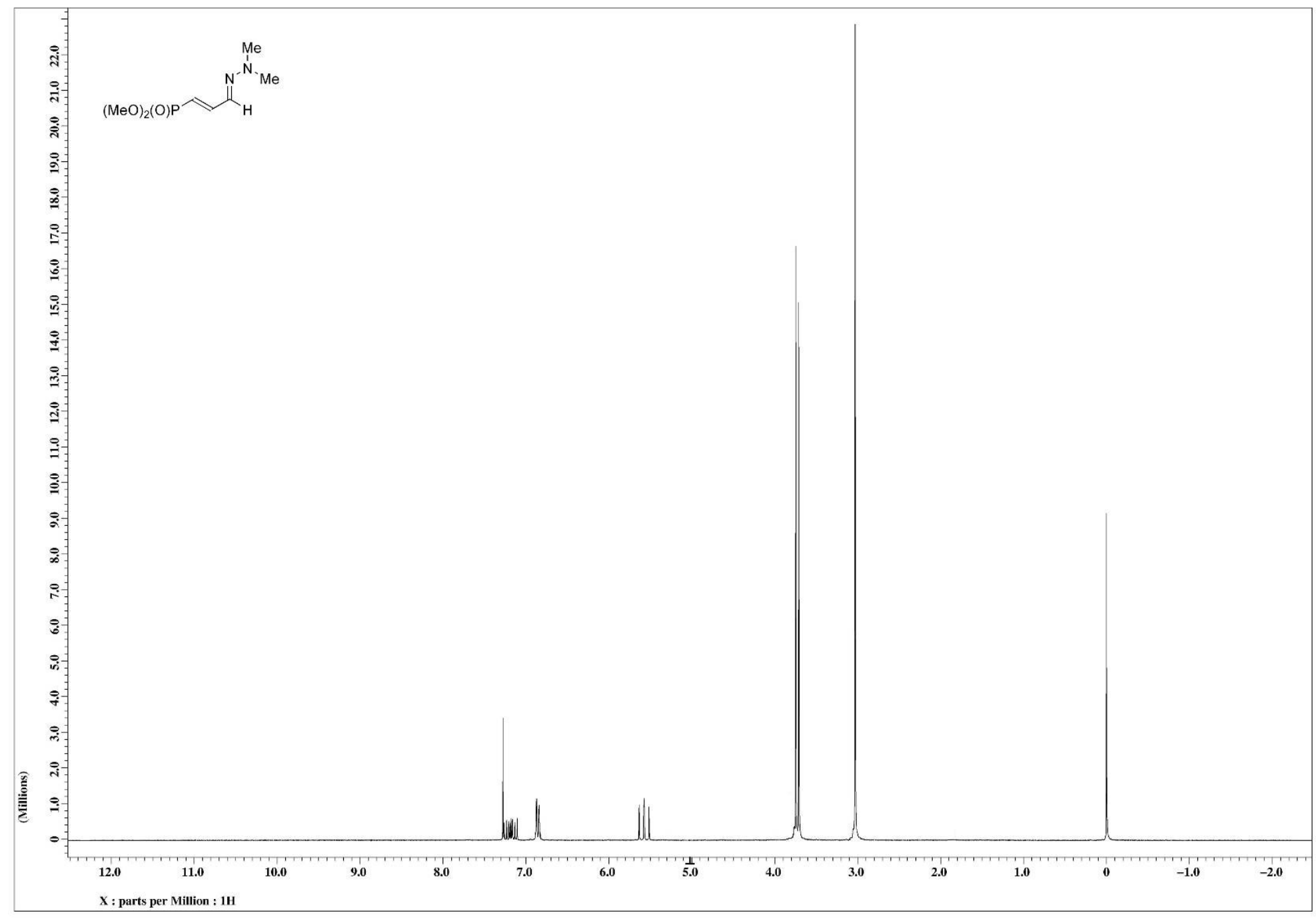

S 40 
Dimethyl (1E,3E)-3-(dimethylhydrazono)prop-1-enylphosphonate 6a $\left({ }^{13} \mathrm{C}-\mathrm{NMR}\right.$ : $\left.75 \mathrm{MHz}, \mathrm{CDCl}_{3}\right)$

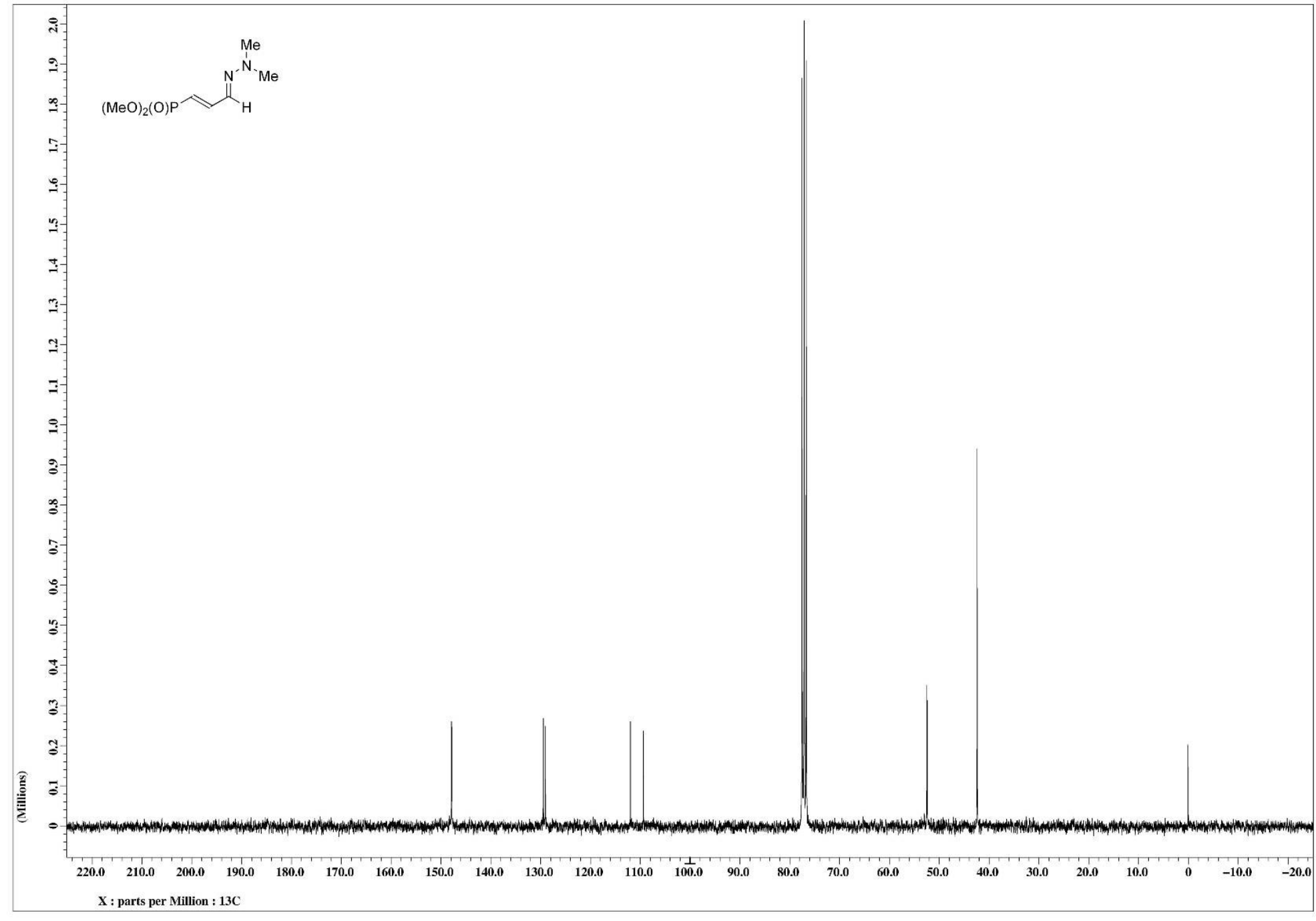


Diethyl (1E,3E)-3-(dimethylhydrazono)prop-1-enylphosphonate $6 \mathrm{~b}\left({ }^{1} \mathrm{H}-\mathrm{NMR}\right.$ : $\left.300 \mathrm{MHz}, \mathrm{CDCl}_{3}\right)$

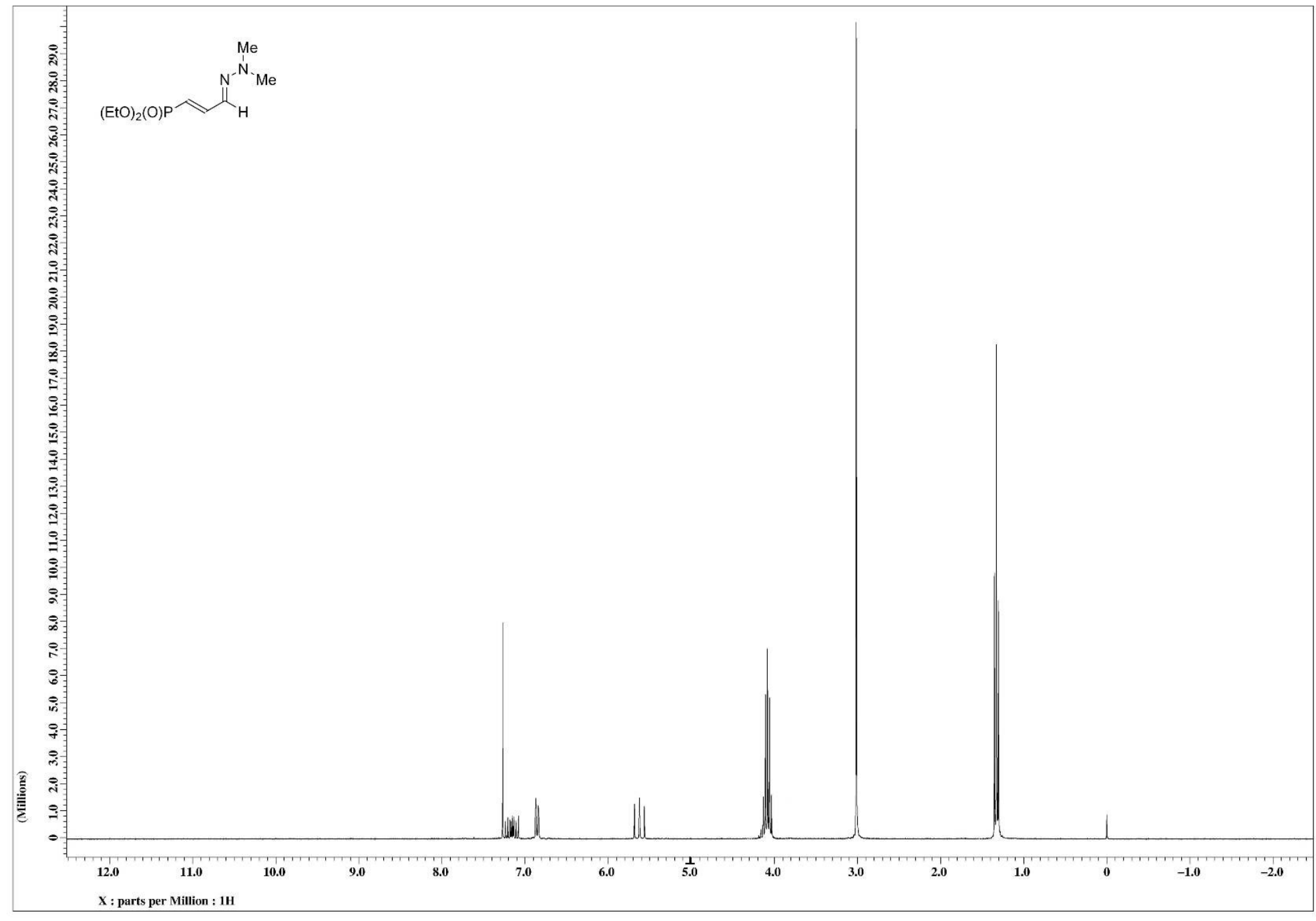


Diethyl (1E,3E)-3-(dimethylhydrazono)prop-1-enylphosphonate $6 \mathrm{~b}\left({ }^{13} \mathrm{C}-\mathrm{NMR}\right.$ : $\left.75 \mathrm{MHz}, \mathrm{CDCl}_{3}\right)$

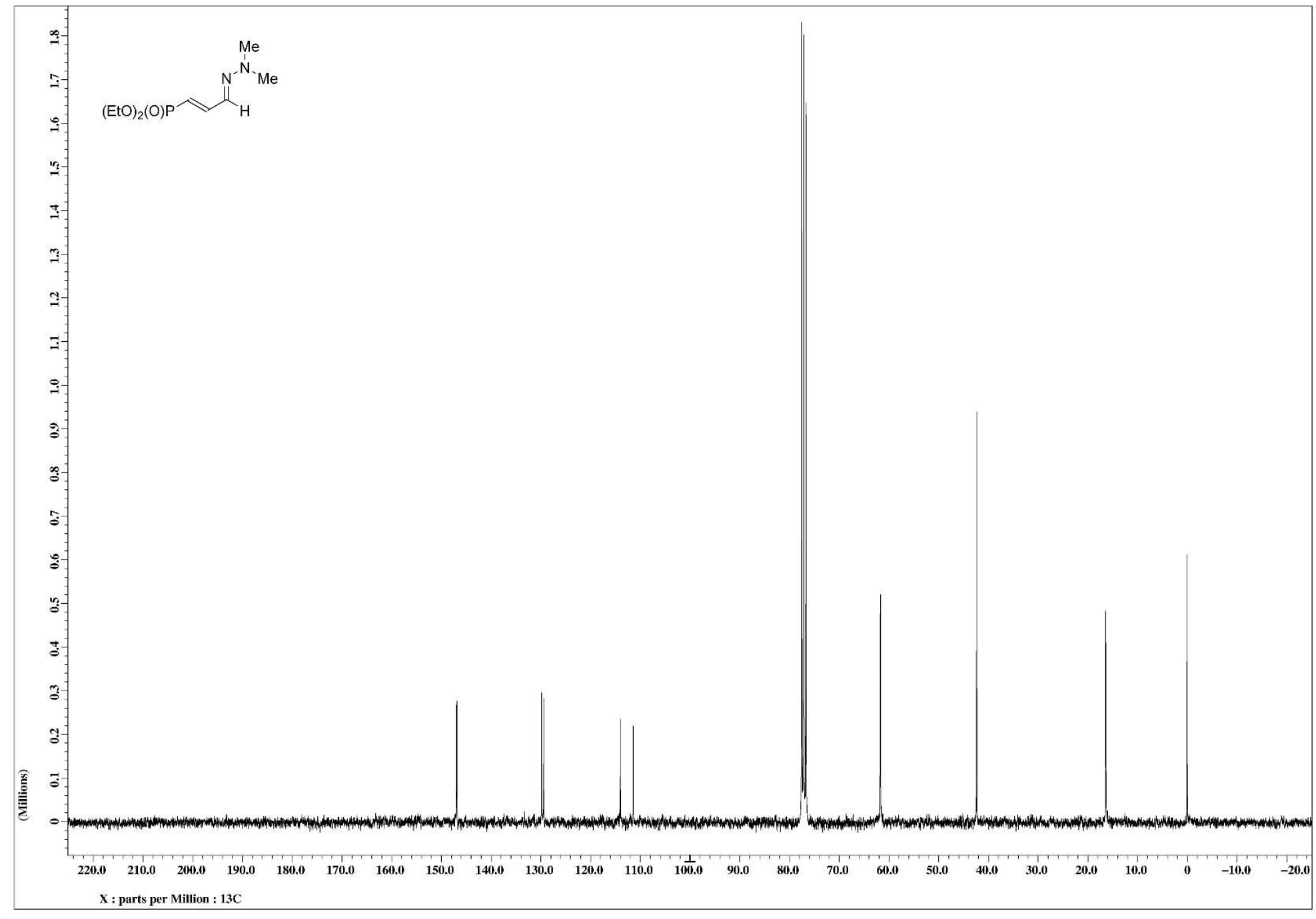


Dimethyl [1,3-bis(dimethoxyphosphoryl)-1-isopropylaminopropyl]phosphonate 9a ( ${ }^{1} \mathrm{H}-\mathrm{NMR}$ : $\left.300 \mathrm{MHz}, \mathrm{CDCl}_{3}\right)$

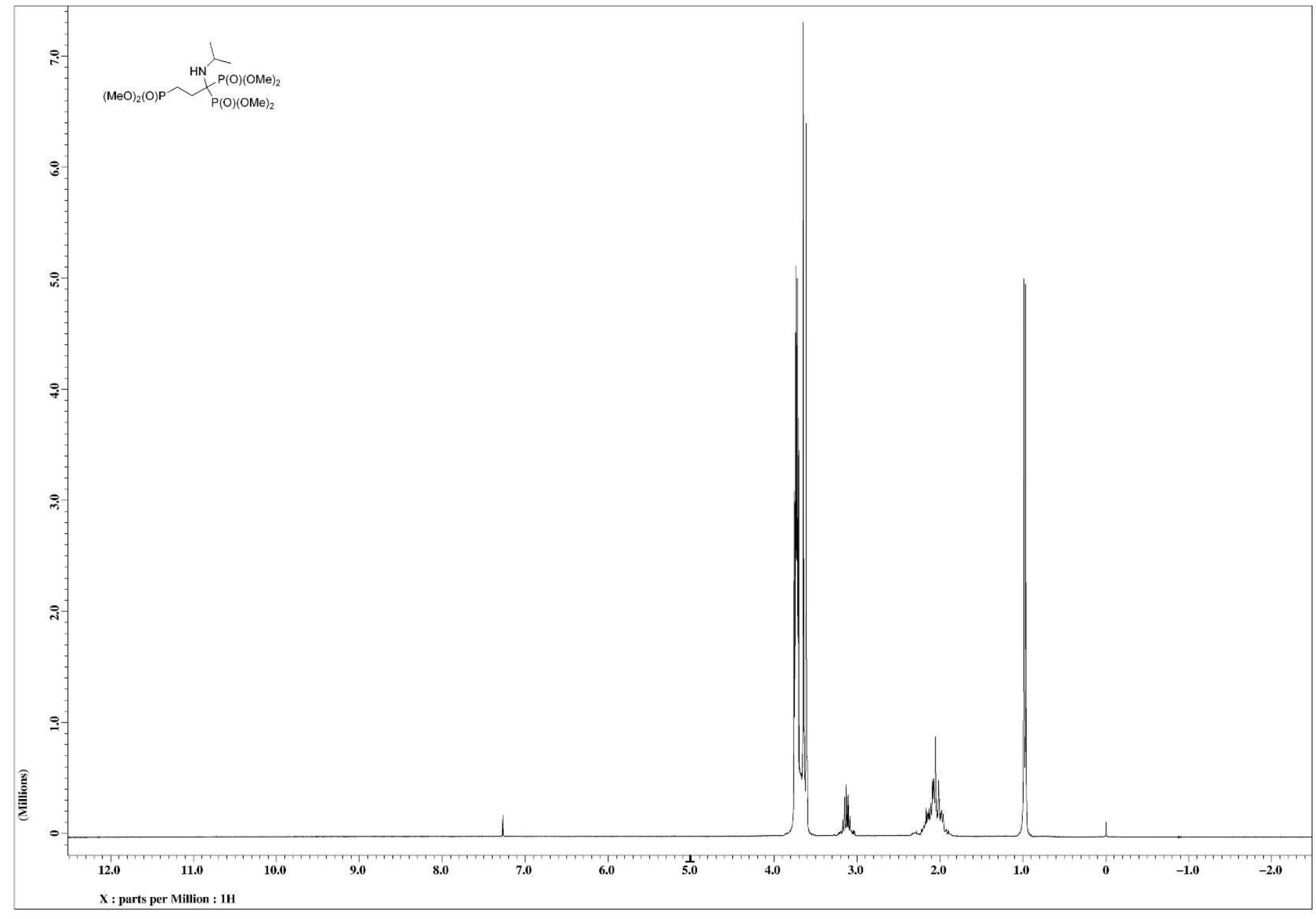


Dimethyl [1,3-bis(dimethoxyphosphoryl)-1-isopropylaminopropyl]phosphonate 9a $\left({ }^{13} \mathrm{C}-\mathrm{NMR}\right.$ : $\left.75 \mathrm{MHz}, \mathrm{CDCl}_{3}\right)$

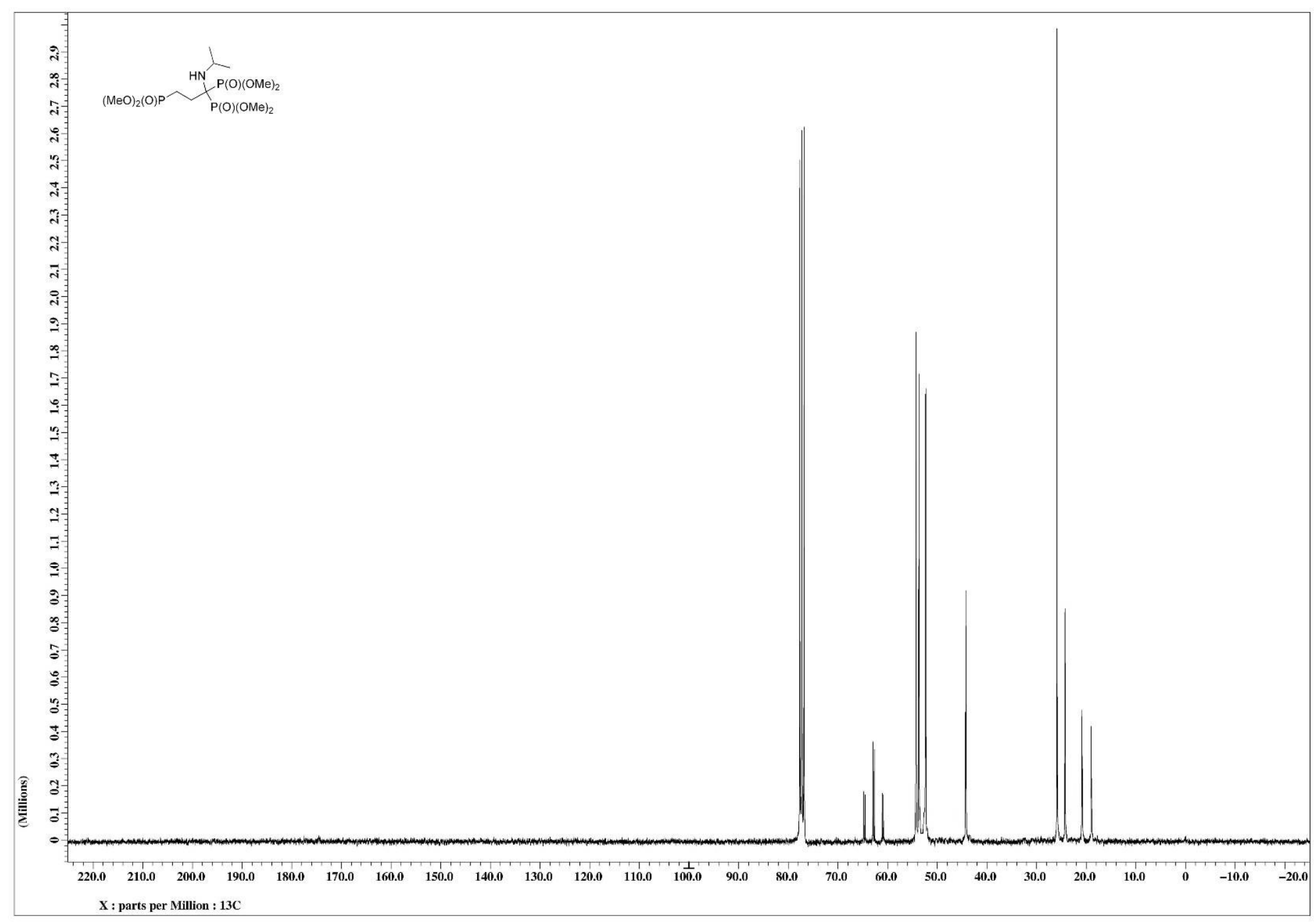


Dimethyl [1,3-bis(dimethoxyphosphoryl)-1-sec-butylaminopropyl]phosphonate 9b $\left({ }^{1} \mathrm{H}-\mathrm{NMR}\right.$ : $\left.300 \mathrm{MHz}, \mathrm{CDCl}_{3}\right)$

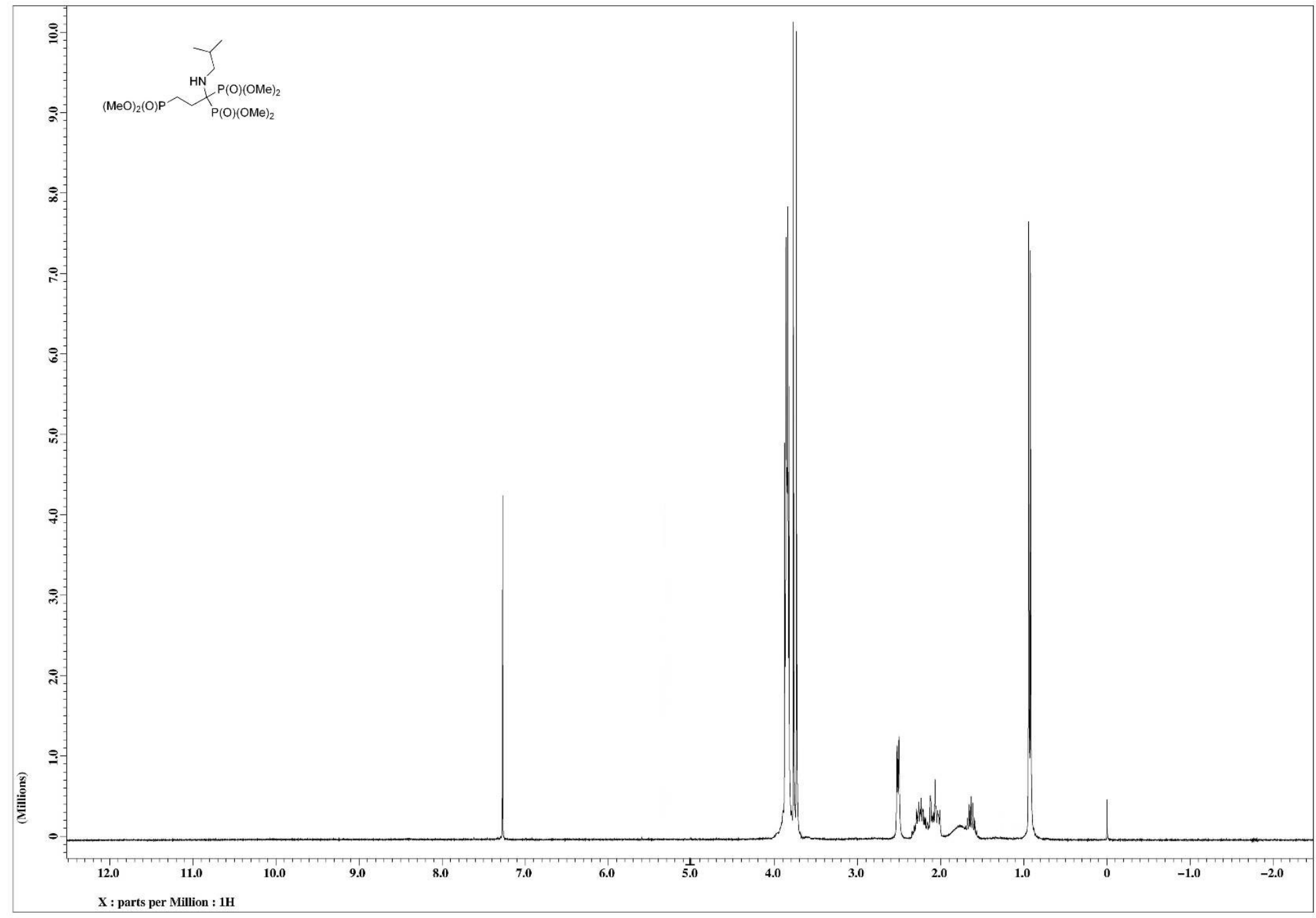

S46 
Dimethyl [1,3-bis(dimethoxyphosphoryl)-1-sec-butylaminopropyl]phosphonate $9 \mathrm{~b}\left({ }^{13} \mathrm{C}-\mathrm{NMR}\right.$ : $\left.75 \mathrm{MHz}, \mathrm{CDCl}_{3}\right)$

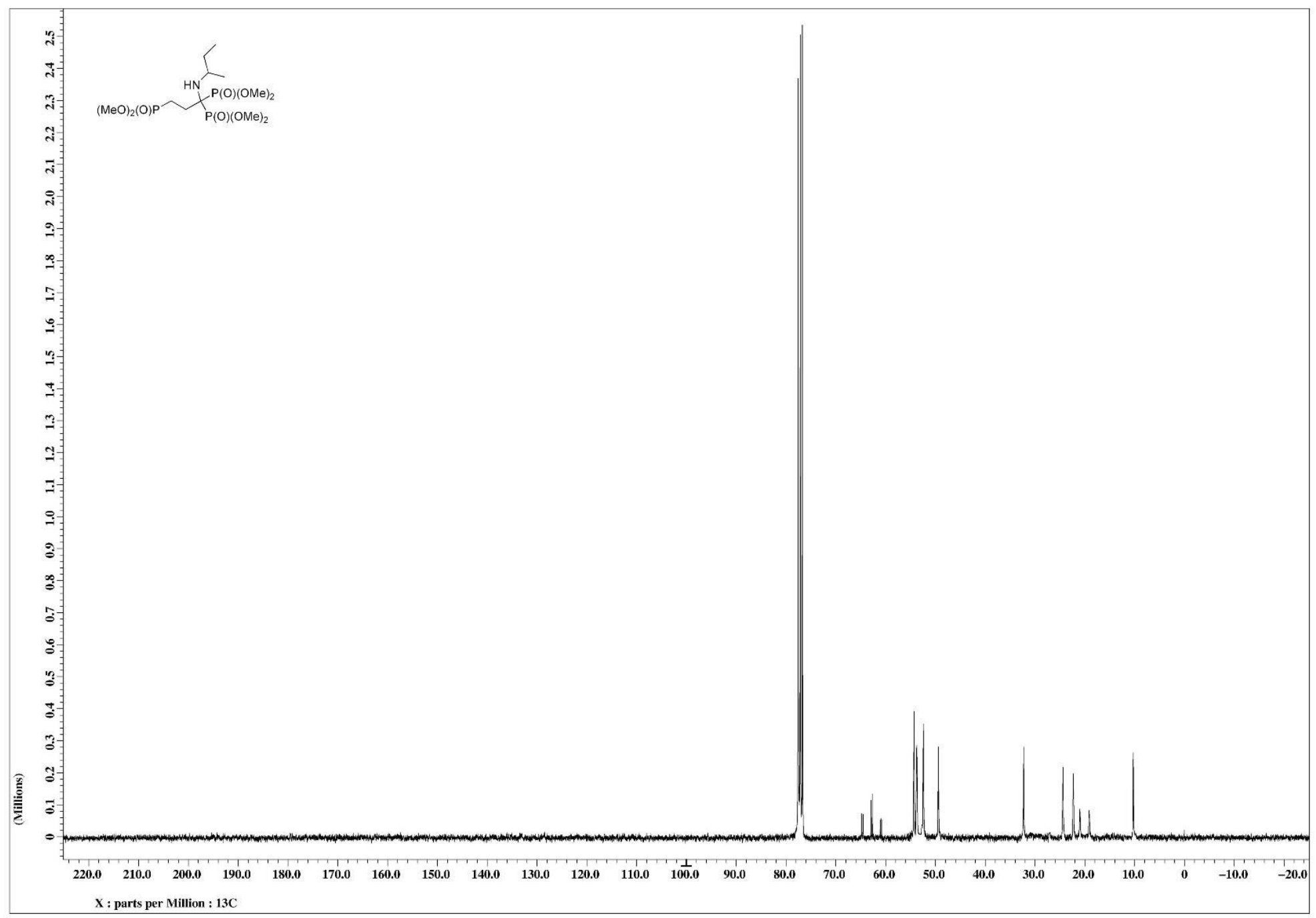

S 47 
Dimethyl [1,3-bis(dimethoxyphosphoryl)-1-isobutylaminopropyl]phosphonate 9c $\left({ }^{1} \mathrm{H}-\mathrm{NMR}\right.$ : $\left.300 \mathrm{MHz}, \mathrm{CDCl}_{3}\right)$

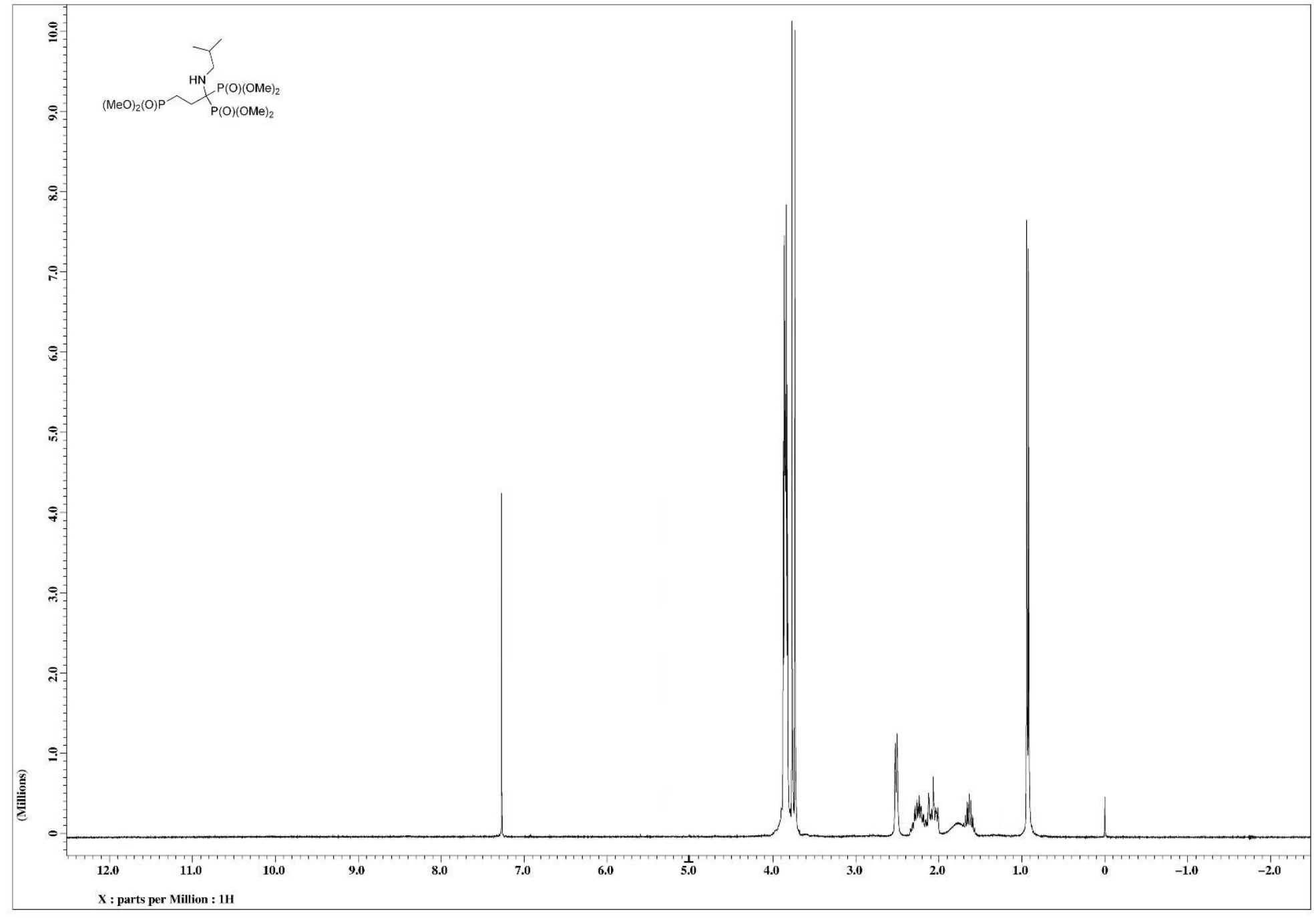

S 48 
Dimethyl [1,3-bis(dimethoxyphosphoryl)-1-isobutylaminopropyl]phosphonate 9c $\left({ }^{13} \mathrm{C}-\mathrm{NMR}\right.$ : $\left.75 \mathrm{MHz}, \mathrm{CDCl}_{3}\right)$

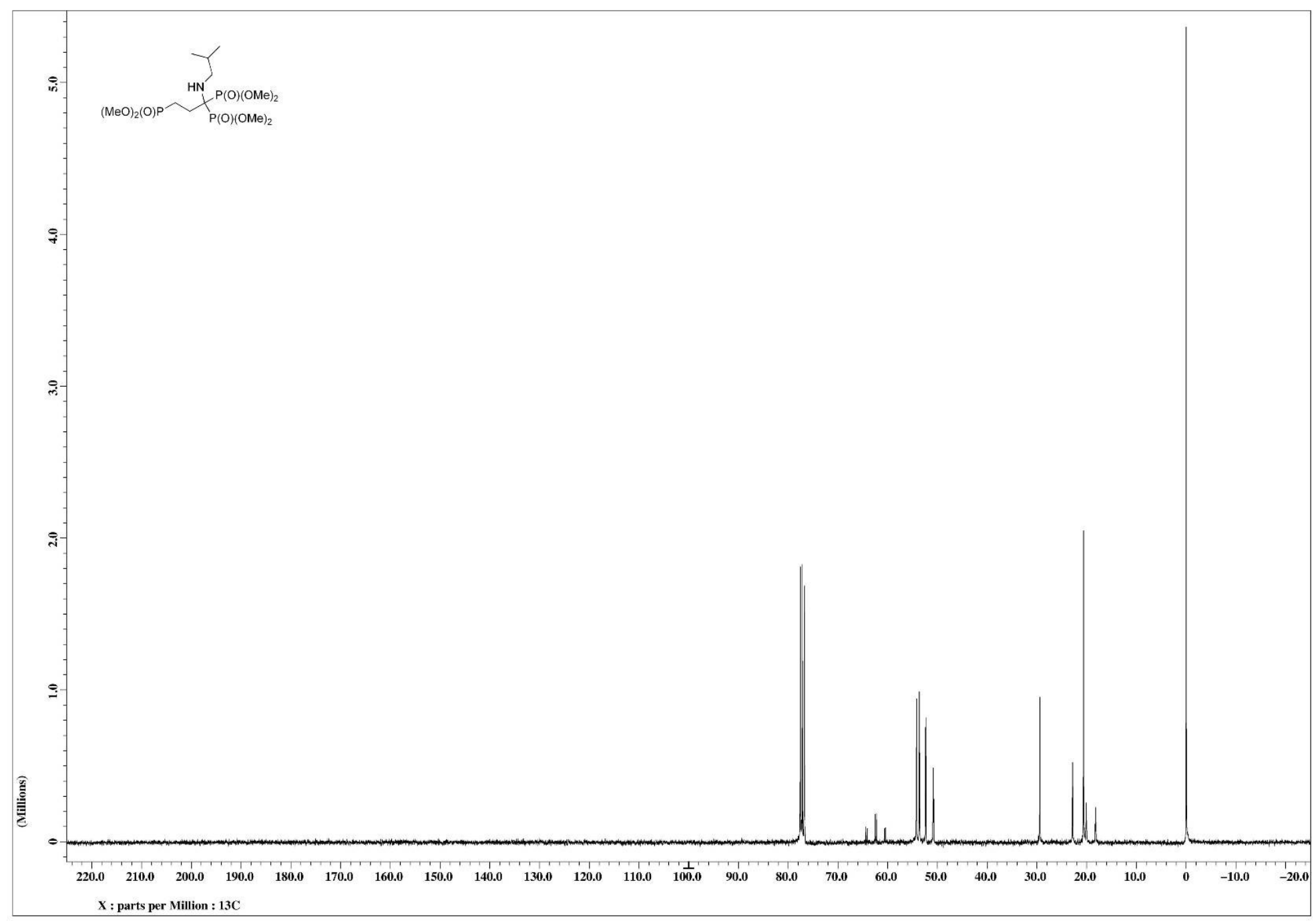


Dimethyl [1,3-bis(dimethoxyphosphoryl)-1-cyclohexylaminopropyl]phosphonate 9d ( ${ }^{1} \mathrm{H}-\mathrm{NMR}$ : $\left.300 \mathrm{MHz}, \mathrm{CDCl}_{3}\right)$

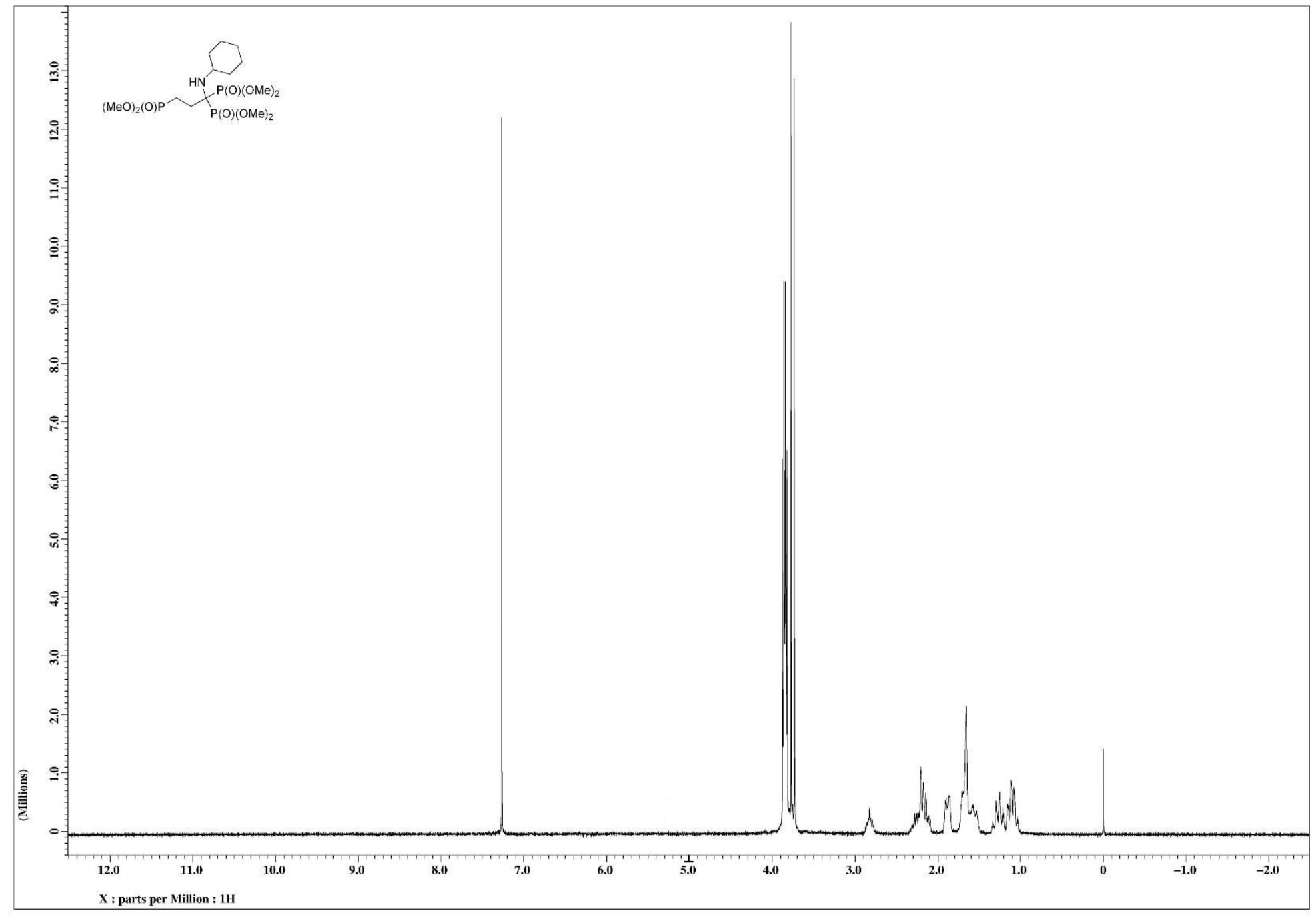

S50 
Dimethyl [1,3-bis(dimethoxyphosphoryl)-1-cyclohexylaminopropyl]phosphonate 9d $\left({ }^{13} \mathrm{C}-\mathrm{NMR}\right.$ : $\left.75 \mathrm{MHz}, \mathrm{CDCl}_{3}\right)$

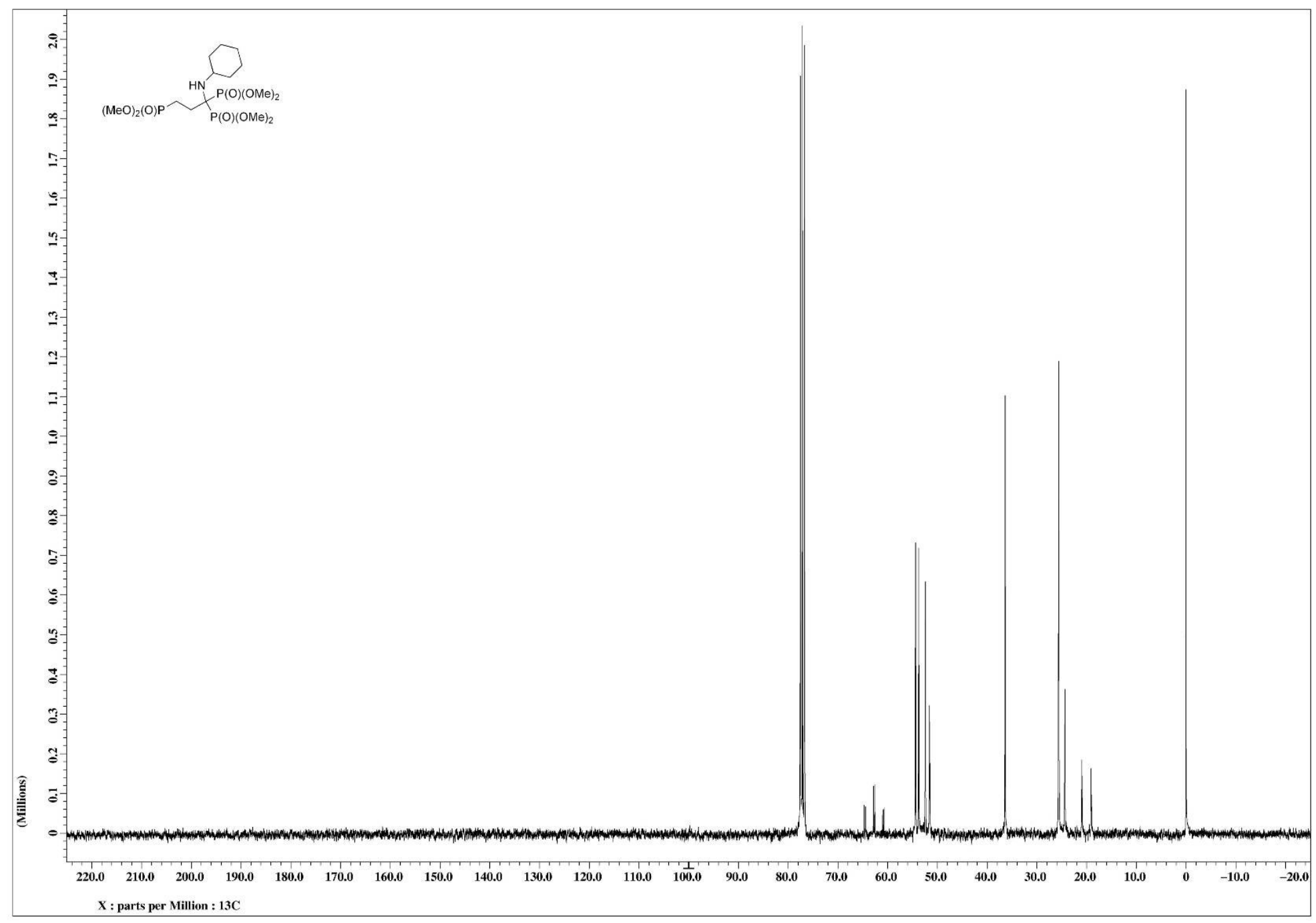


Dimethyl [1,3-bis(dimethoxyphosphoryl)-1-benzylaminopropyl]phosphonate 9e ( ${ }^{1} \mathrm{H}-\mathrm{NMR}$ : $\left.300 \mathrm{MHz}, \mathrm{CDCl}_{3}\right)$

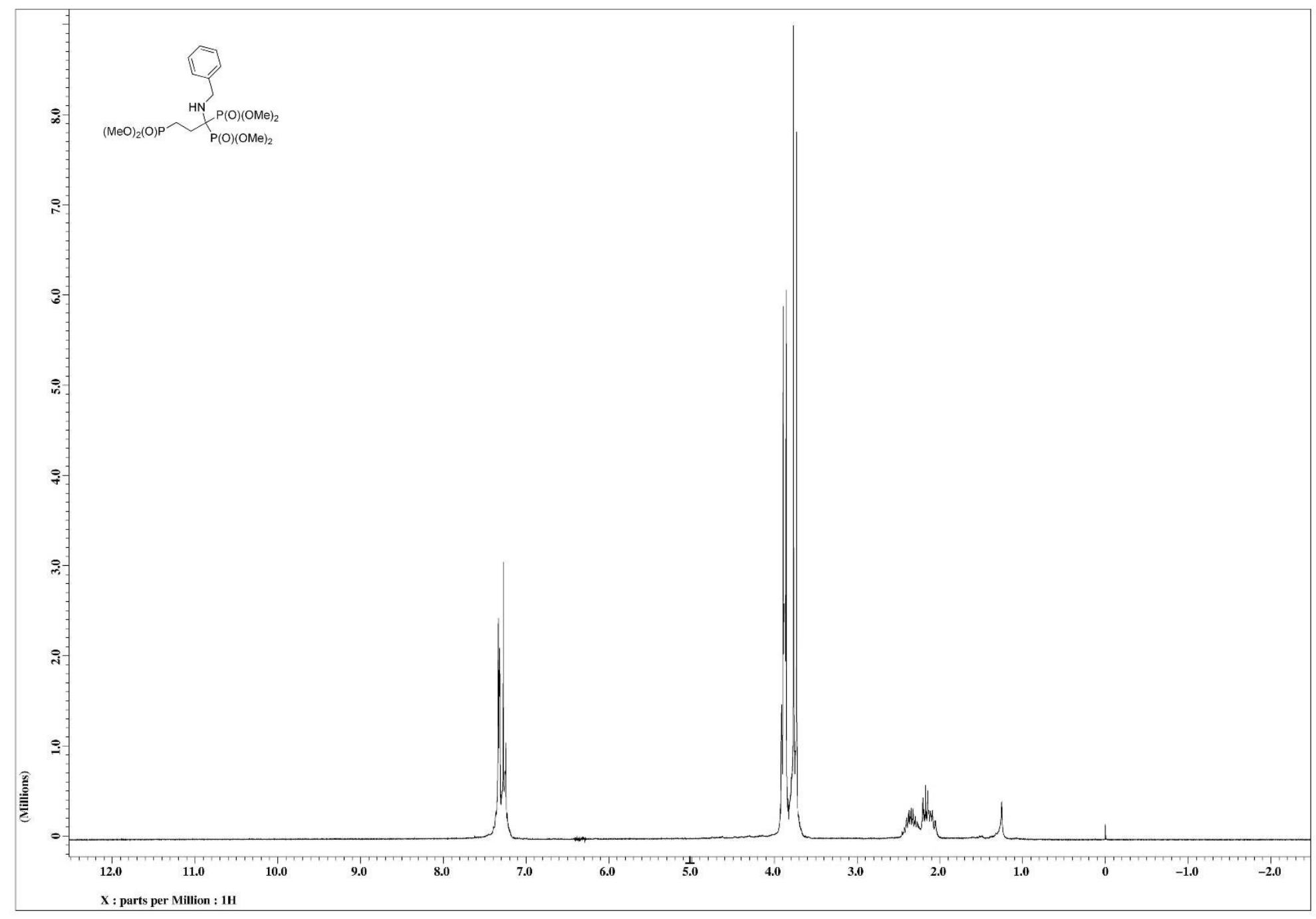


Dimethyl [1,3-bis(dimethoxyphosphoryl)-1-benzylaminopropyl]phosphonate 9e $\left({ }^{13} \mathrm{C}-\mathrm{NMR}\right.$ : $\left.75 \mathrm{MHz}, \mathrm{CDCl}_{3}\right)$

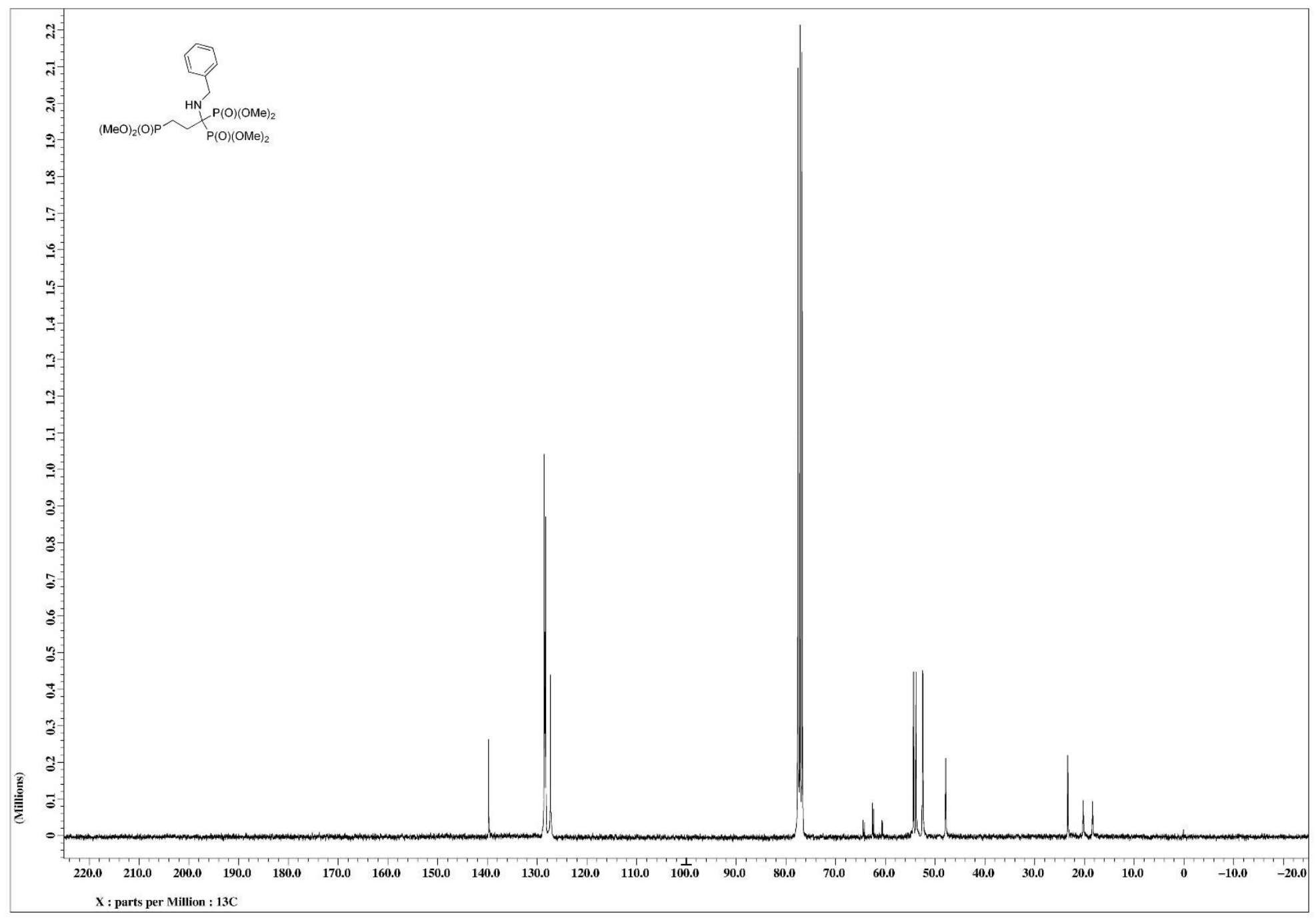

S 53 
Diethyl [1,3-bis(diethoxyphosphoryl)-1-isopropylaminopropyl]phosphonate 9f ( ${ }^{1} \mathrm{H}-\mathrm{NMR}$ : $\left.300 \mathrm{MHz}, \mathrm{CDCl}_{3}\right)$

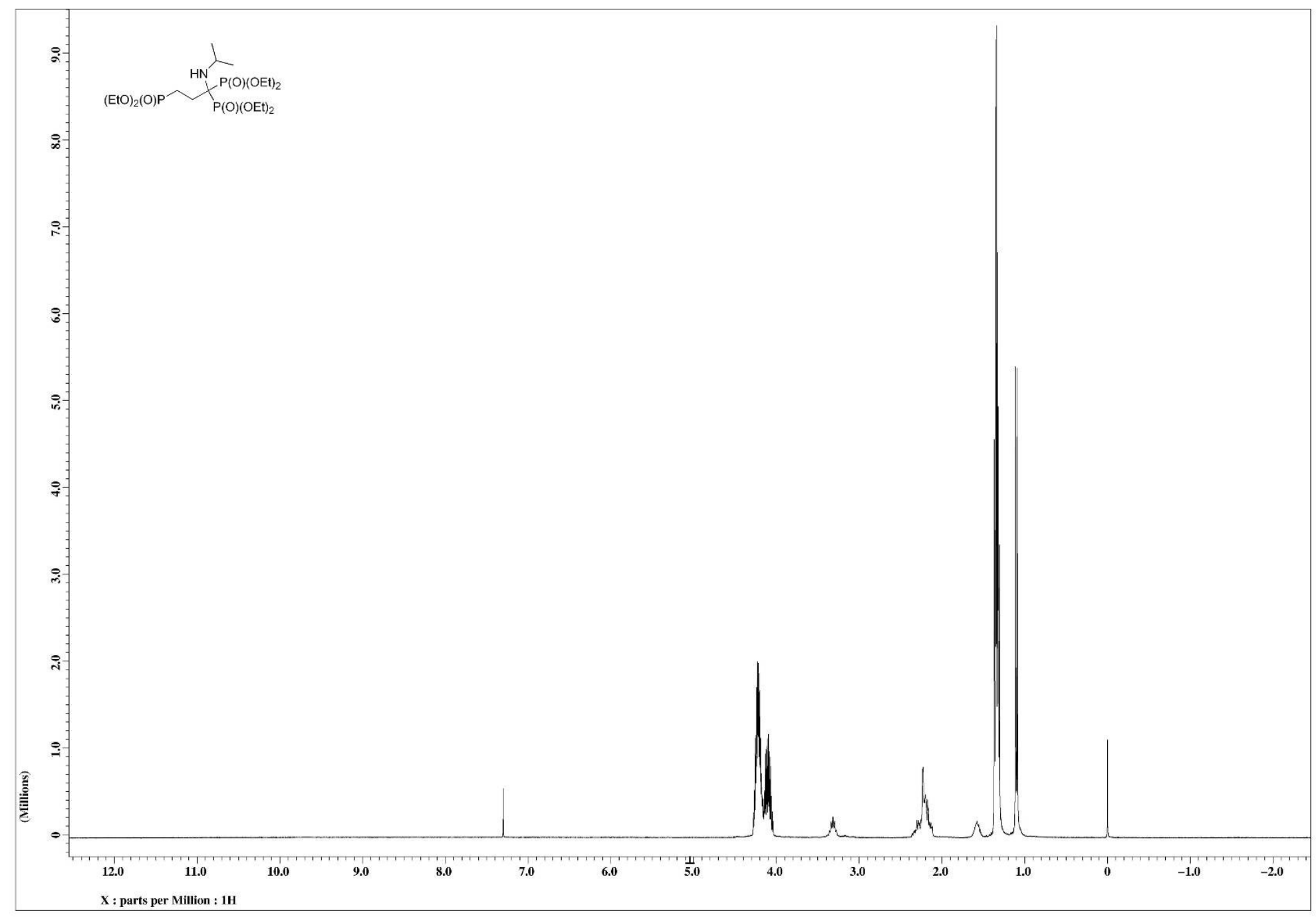

S54 
Diethyl [1,3-bis(diethoxyphosphoryl)-1-isopropylaminopropyl]phosphonate 9f $\left({ }^{13} \mathrm{C}-\mathrm{NMR}\right.$ : $\left.75 \mathrm{MHz}, \mathrm{CDCl}_{3}\right)$

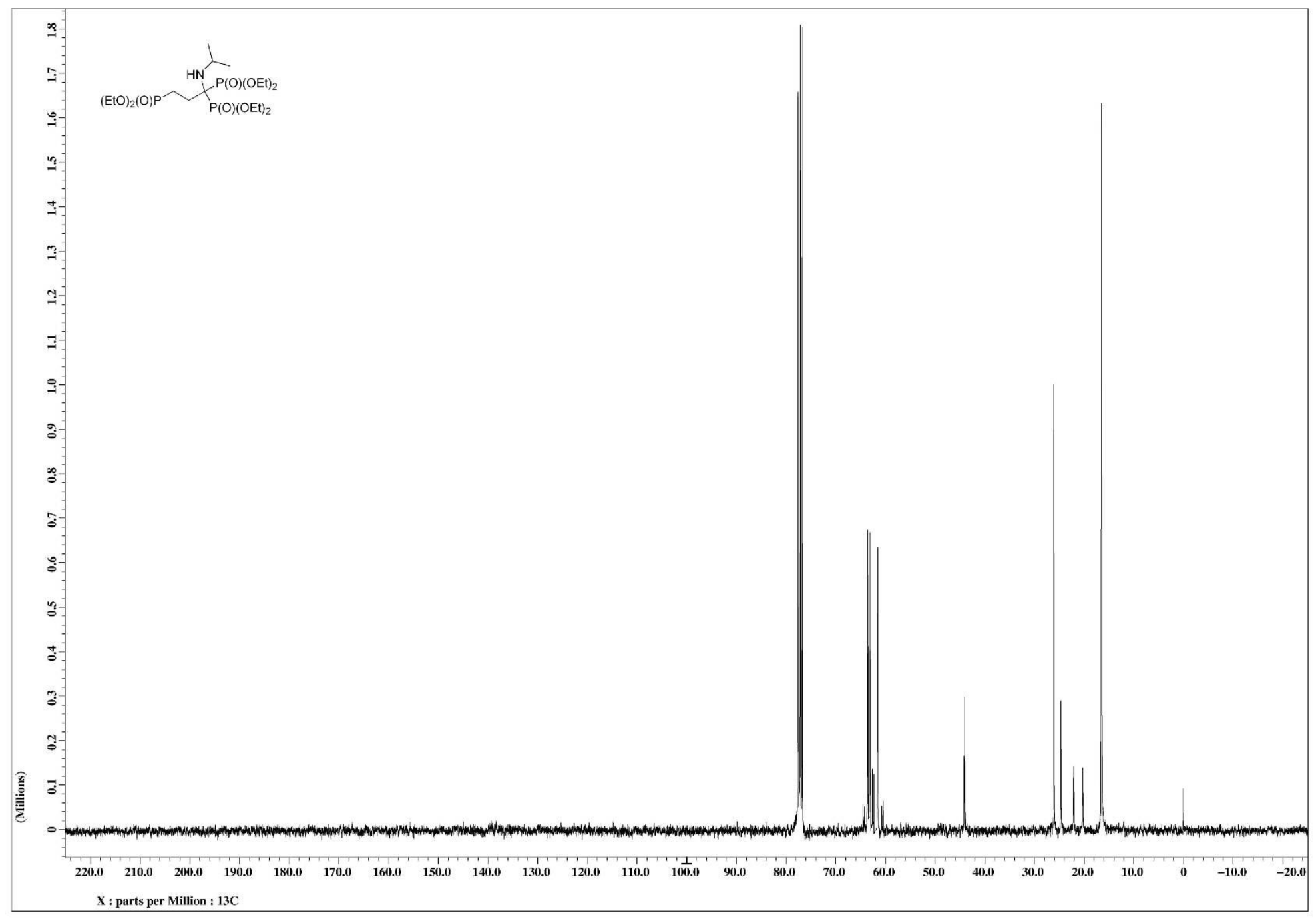

S 55 
Diethyl [1,3-bis(diethoxyphosphoryl)-1-sec-butylaminopropyl]phosphonate 9g ( ${ }^{1} \mathrm{H}-\mathrm{NMR}$ : $\left.300 \mathrm{MHz}, \mathrm{CDCl}_{3}\right)$

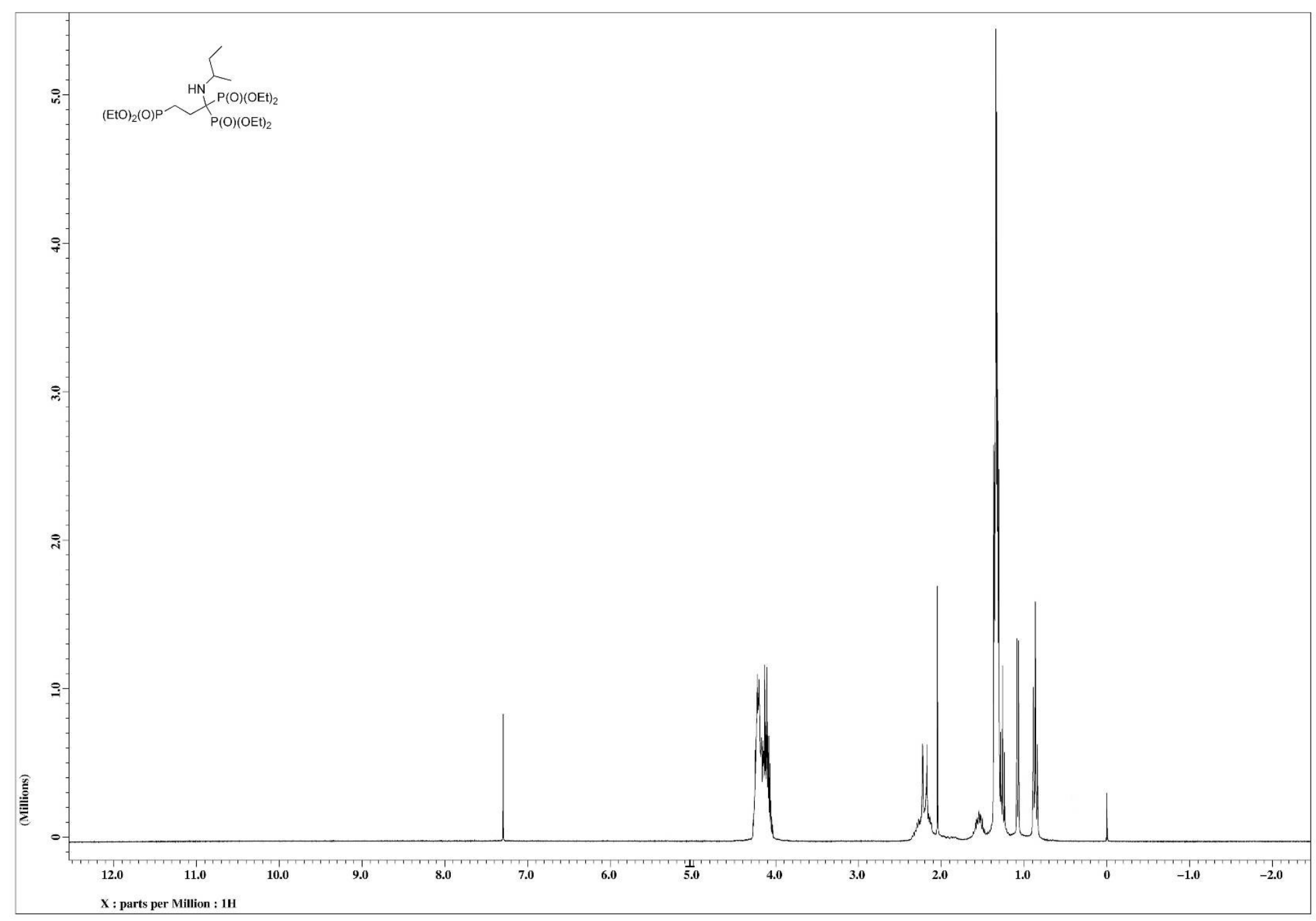

S 56 
Diethyl [1,3-bis(diethoxyphosphoryl)-1-sec-butylaminopropyl]phosphonate $9 \mathrm{~g}\left({ }^{13} \mathrm{C}-\mathrm{NMR}\right.$ : $\left.75 \mathrm{MHz}, \mathrm{CDCl}_{3}\right)$

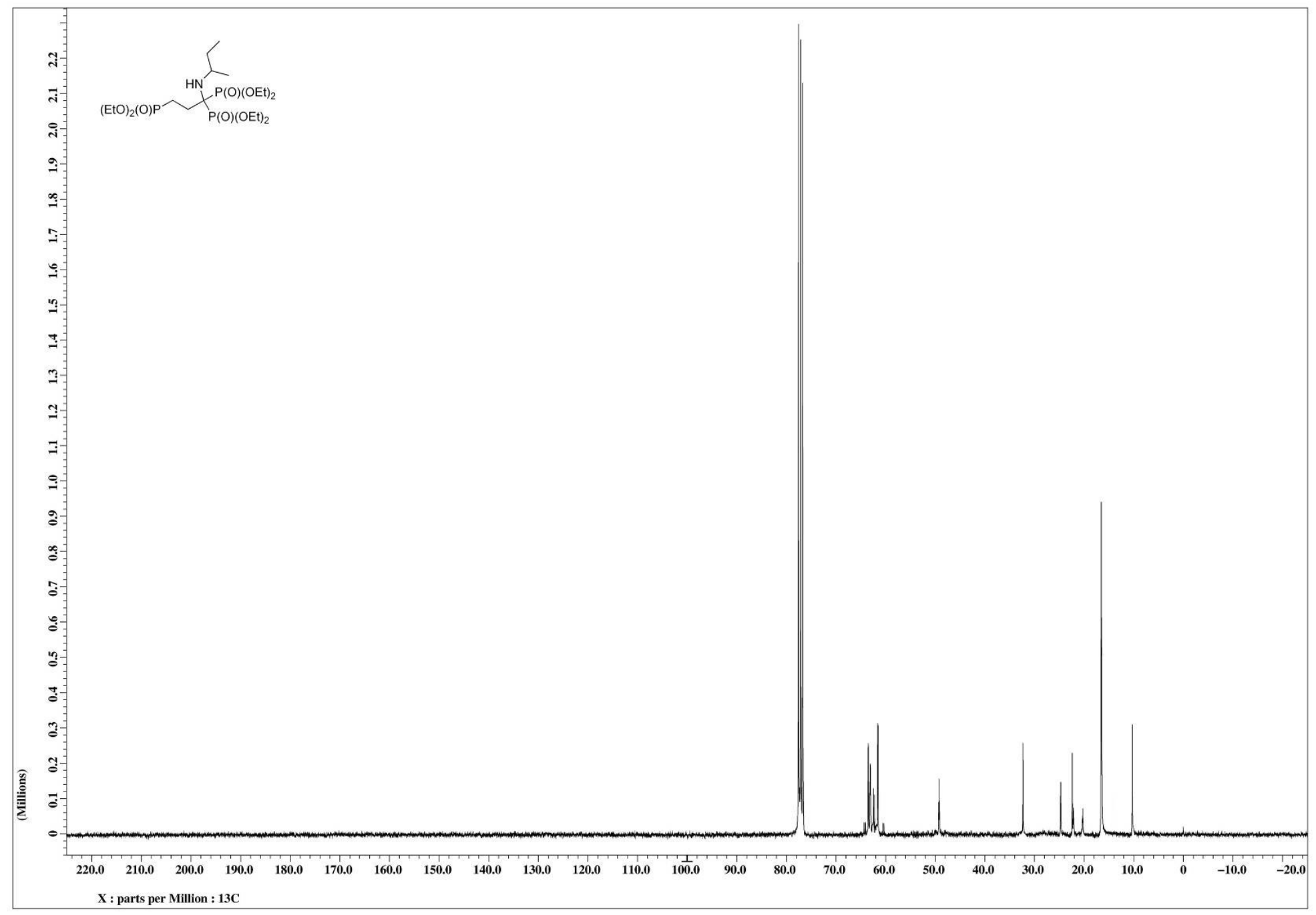


Diethyl [1,3-bis(diethoxyphosphoryl)-1-cyclohexylaminopropyl]phosphonate $9 \mathrm{~h}\left({ }^{1} \mathrm{H}-\mathrm{NMR}\right.$ : $\left.300 \mathrm{MHz}, \mathrm{CDCl}_{3}\right)$

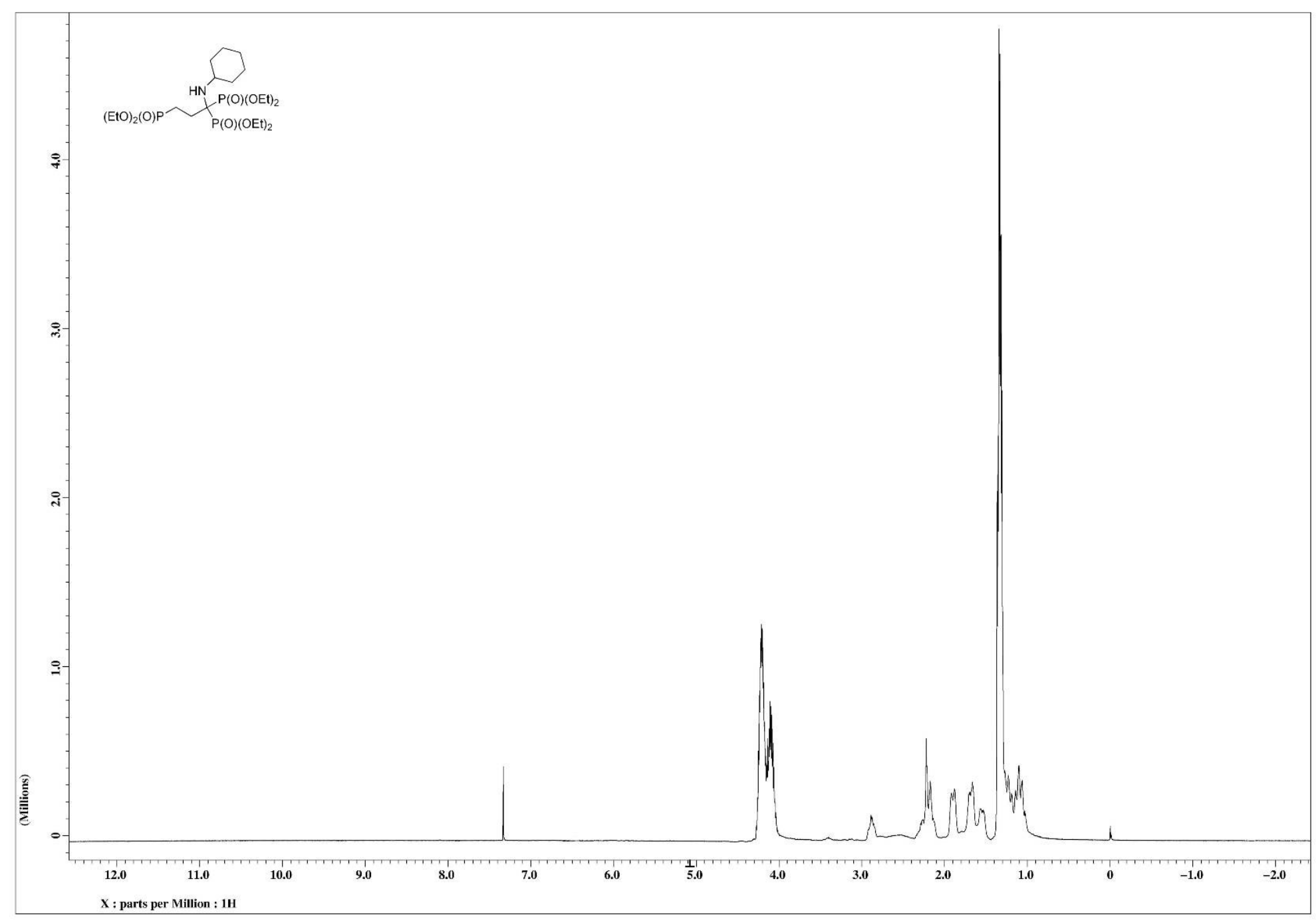

S 58 
Diethyl [1,3-bis(diethoxyphosphoryl)-1-cyclohexylaminopropyl]phosphonate $9 \mathrm{~h}\left({ }^{13} \mathrm{C}-\mathrm{NMR}\right.$ : $\left.75 \mathrm{MHz}, \mathrm{CDCl}_{3}\right)$

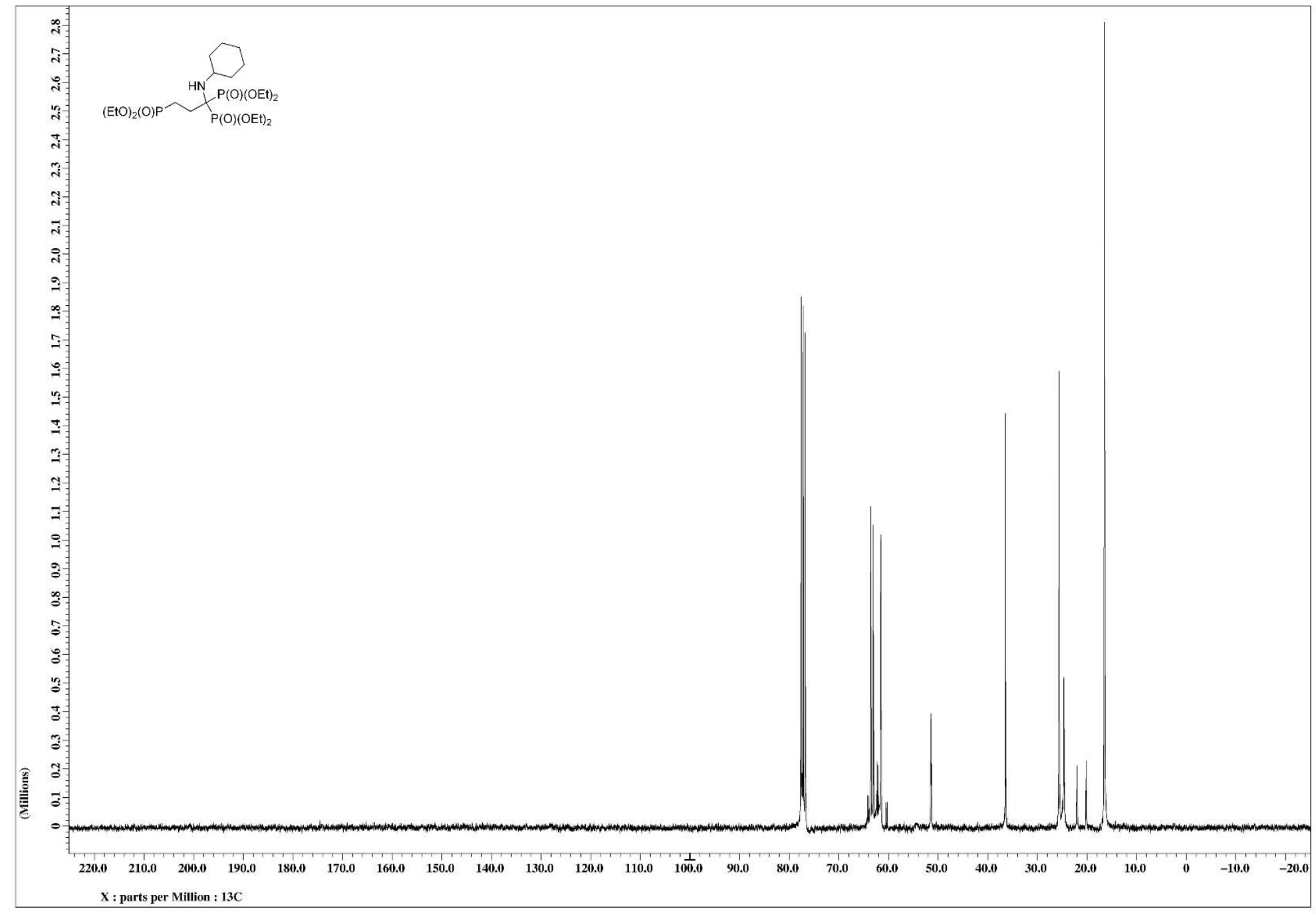


Dimethyl [1-t-butylamino-3,3-bis(dimethoxyphosphoryl)propyl]phosphonate $10\left({ }^{1} \mathrm{H}-\mathrm{NMR}\right.$ : $\left.300 \mathrm{MHz}, \mathrm{CDCl}_{3}\right)$

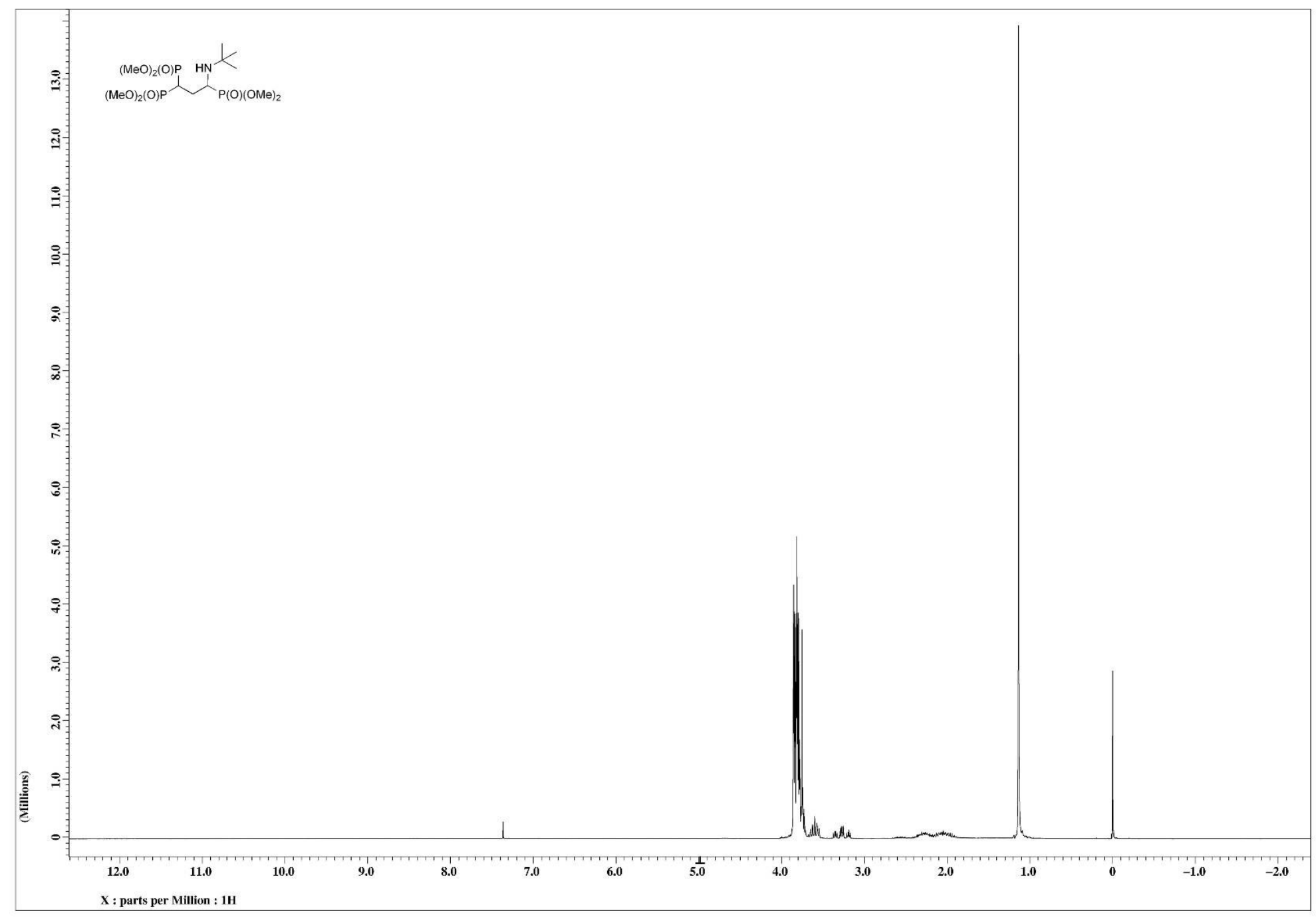


Dimethyl [1-t-butylamino-3,3-bis(dimethoxyphosphoryl)propyl]phosphonate $10\left({ }^{13} \mathrm{C}-\mathrm{NMR}\right.$ : $\left.75 \mathrm{MHz}, \mathrm{CDCl}_{3}\right)$

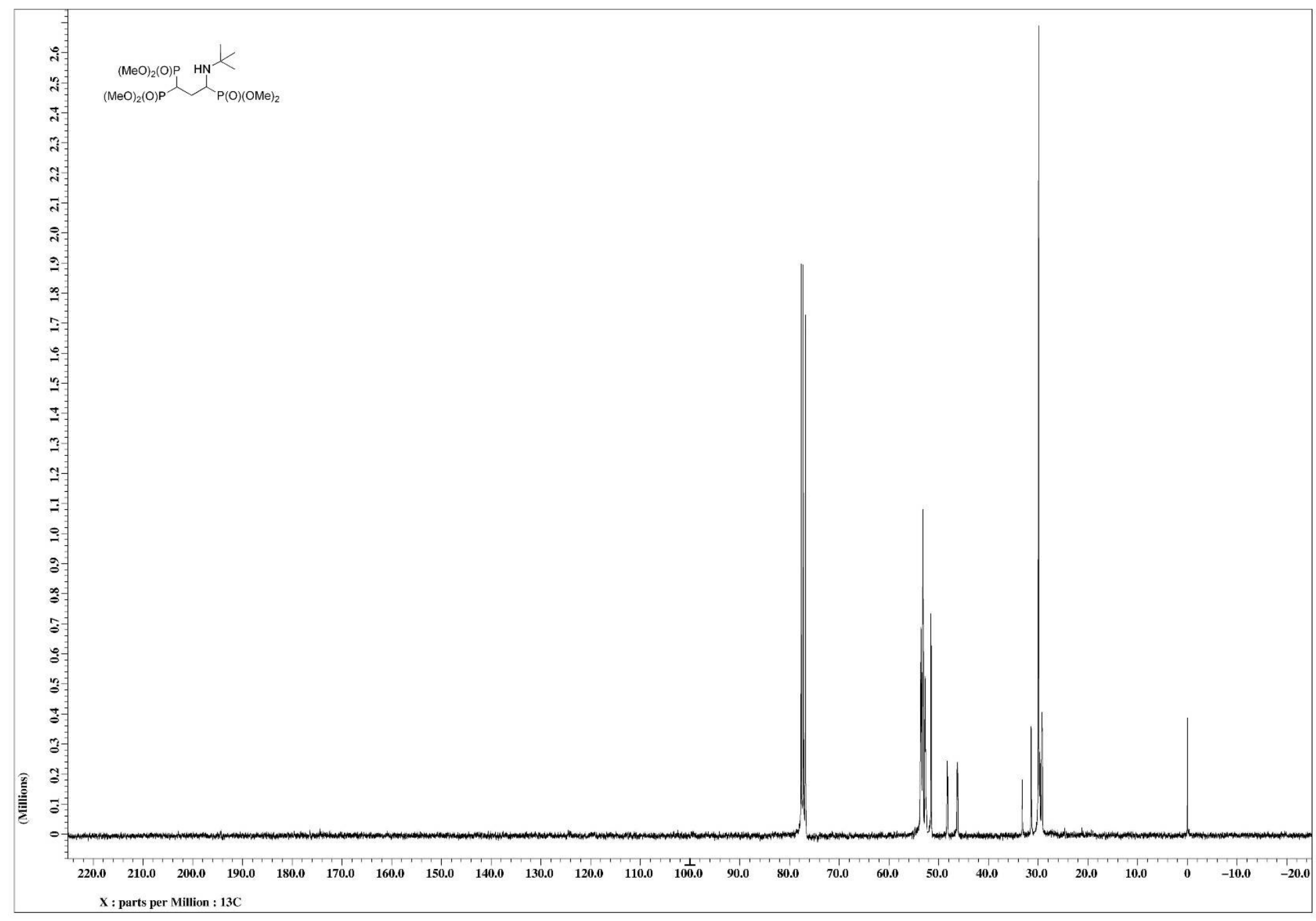

